MARIE-JOSÉE LEPAGE

\title{
LES FACTEURS PROSODIQUES QUI MARQUENT LA PERCEPTION DES FINS DE TOUR DE PAROLE
}

\author{
Mémoire présenté \\ à la Faculté des études supérieures de I'Université Laval \\ comme exigence partielle du programme de maîtrise en linguistique \\ offert à I'Université du Québec à Chicoutimi \\ en vertu d'un protocole d'entente avec IUniversité Laval \\ pour l'obtention du grade de maître ès arts (M.A.)
}

\author{
FACULTEE DES LETIRES \\ UNIVERSITÉ LAVAL \\ QUÉBEC
}

et

\section{DÉPARTEMENT DES ARTS ET LETTRES UNIVERSITÉ DU QUÉBEC À CHICOUTIMI SAGUENAY}

(C) Marie-Josée Lepage, 2009 


\section{Bibliothèque}

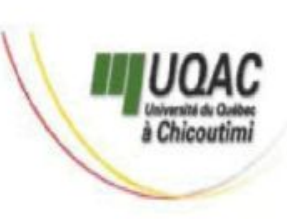

\section{Mise en garde/Advice}

Afin de rendre accessible au plus Motivated by a desire to make the grand nombre le résultat des results of its graduate students' travaux de recherche menés par ses research accessible to all, and in étudiants gradués et dans l'esprit des accordance with the rules règles qui régissent le dépôt et la governing the acceptation and diffusion des mémoires et thèses diffusion of dissertations and produits dans cette Institution, theses in this Institution, the I'Université du Québec à Université du Québec à Chicoutimi (UQAC) est fière de Chicoutimi (UQAC) is proud to rendre accessible une version make a complete version of this complète et gratuite de cette œuvre. work available at no cost to the reader.

L'auteur conserve néanmoins la The author retains ownership of the propriété du droit d'auteur qui copyright of this dissertation or protège ce mémoire ou cette thèse. thesis. Neither the dissertation or Ni le mémoire ou la thèse ni des thesis, nor substantial extracts from extraits substantiels de ceux-ci ne it, may be printed or otherwise peuvent être imprimés ou autrement reproduced without the author's reproduits sans son autorisation. permission. 


\section{RÉSUMÉ}

La présente étude s'intéresse principalement aux éléments prosodiques, c'est-àdire lintonation, la pause et l'intensité, utilisés dans une conversation afin de comprendre comment les locuteurs les utilisent pour signaler la fin de leurs tours de parole. Pour ce faire, nous avons utilisé un corpus composé de 20 tours de parole longs tirés d'une conversation spontanée entre deux étudiants québécois de niveau universitaire.

Pour réaliser cette expérimentation, nous avons procédé en deux grandes étapes. Tout d'abord, une analyse auditive a été faite afin d'identifier les facteurs présents à la fin et à l'intérieur des tours de parole. Par la suite, les facteurs prosodiques ont été soumis à une analyse instrumentale plus poussée. Afin d'avoir un portrait un peu plus fidèle à la réalité, des éléments syntaxiques et sémanticopragmatiques ont aussi été étudiés. Les résultats obtenus montrent que l'utilisation des différents indices étudiés est assez partagée. Il semblerait en effet que les locuteurs de notre étude aient tendance à utiliser un ensemble d'éléments pour signaler la fin de leurs tours de parole plutôt que d'utiliser un élément de façon prédominante. Contrairement à l'idée généralement répandue, la production d'une pause longue accompagnée d'une descente de fréquence et d'intensité ne serait pas un indice suffisant en oral spontané. Les facteurs prosodiques auraient besoin d'être complétés par des indices syntaxiques et sémantico-pragmatiques pour être efficaces. 


\section{REMERCIEMENTS}

ENFIN!!! Je crois que ce mot ne peut pas être plus à propos qu'ici. OUF! Tant de temps s'est écoulé entre le début de mon tout premier séminaire et le dépôt initial, mais qui marque tout de même l'etape non moins finale de cette course d'endurance «à obstacles». On ne peut même plus ici parler d'accouchement, c'est réellement une délivrance.

Après tout ce temps, qui dois-je remercier? Avec ma mémoire vive de $2 \mathrm{Ko}$, je ne suis plus sare de me souvenir de tout le monde. Alors, je le fais tout de suite, merci à ceux que j'oublierai dans les prochaines lignes.

Mon Dieu, par qui commencer? Vous avez tous joué un rôle important. Tout $d^{\prime}$ abord, tiens, merci à celle qui s'y attend peut-être le moins, Monique, pour toutes les sessions de thérapie, de "counselling" et d'orientation que tu m'as si gentiment données.

Merci à Julie et à Stéphanie qui, même si elles m’ont désertée depuis déjà quelques années en tant que «étudiantes de maîtrise», ont su piquer mon orgueil et m'ont donné une partie de l'énergie nécessaire pour continuer. Merci aussi à Gilles pour ses conseils linguistiques sans oublier nos sessions de défoulement qui ont fait tant de bien. 
Un gros merci à mon directeur, M. Dolbec, qui aurait tellement pu m'abandonner au fil des années. Après tout ce temps, il a réussi à avoir encore confiance en moi et a trouver les bons encouragements. Merci aussi pour votre ceil critique qui a toujours su me ramener à un travail plus... scientifique.

Un merci tout special à mes parents qui m'ont toujours soutenue tout au long de ces nombreuses années et tout particulièrement à toi maman, pour être venue si généreusement garder à la maison pour que je puisse « finir un jour! » Maman! Je l'ai finie, ma maîtrise!

Un énorme, énorme merci à mon amour David sans qui je n'aurais probablement jamais fini cette satanee maîtrise. Tu as toujours su trouver les bons mots pour m'encourager ou pour tout simplement me remettre sur le bon chemin. Tu es une partie très importante de cette réussite. Quand on sera vieux, on pourra dire qu'on en a réussi des belles 'tites choses ensemble.

Merci aussi à mes 3 petits amours, Philippe, Etienne et Raphaël, vous avez été mes inspirations, mes bouées. Je pouvais toujours compter sur vous trois pour avoir des beaux callins énergisants, pour me rappeler que ça valait la peine de persévérer. Maman sera beaucoup plus disponible maintenant! 
TABLE DES MATIERES

RESUME i

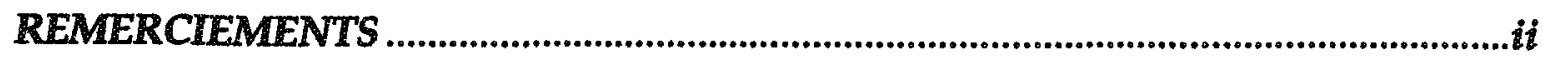

TABLE DES MATIËRES ........................................................................................

LISTE DES GRAPHIQUE ET TABLEAUX .............................................................ix

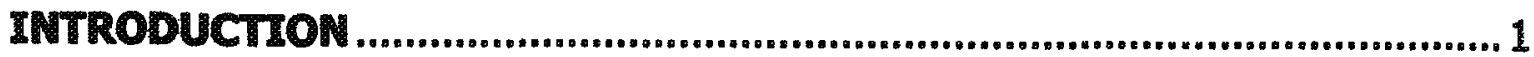

CHAPITRE 1 : PROBLÉMATHQUE ....................................................... 7

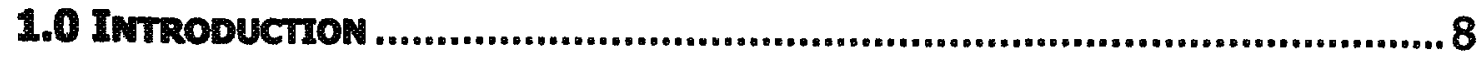

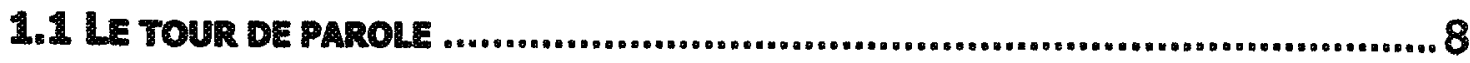

1.2 Un travaIL DE PRÉCURSEUR : DUNCAN (1972) ............................... 10

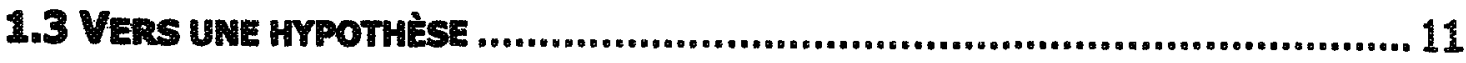

CHAPITRE 2 : ÉTAT DE LA QUESTION ............................................... 14

2.0 INTRODUCTION ......................................................................... 15

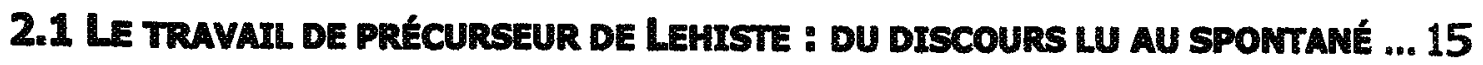

2.2 LES TRAVAUX PLUS RÉCENTS SUR LE SPONTANÉ 19 
2.2.1 Les indices phonétiques: les travaux de Swerts............... 20

2.2.1.1 LES INDICES PHONÉTZUES DANS LE MONOLOGUE ....................... 20

2.2.1.1.1 Le contour intonatif des monologues dinstructions . 22

2.2.1.1.2 L'organisation en topiques...................................... 24

2.2.1.2 LES INDICES PHONÉTIQUES DANS IE DIALOGUE.......................... 27

2.2.2 Les indices non prosodiques .............................................. 30

2.2.2.1 LES FACTEURS SYNTAXIQUES ............................................. 30

2.2.2.2 LES FACTEURS SÉMANTICO-PRAGMATIQUES ............................. 32

CHAPITRE 3 : MÉTHODOLOGIE.......................................................... 37

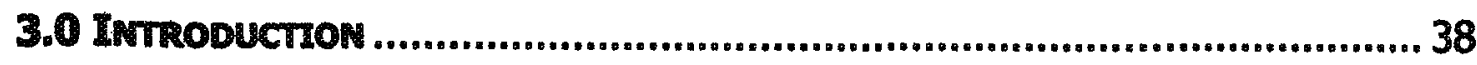

3.1 PRÉSEMTATION DU CORPUS ......................................................... 38

3.1.1 Choix du corpus ................................................................. 39

3.1.2 Constitution d'un corpus ............................................... 40

3.2 SELECTION DES TOURS DE PAROLE LONGS.......................................... 41

3.2.1 Le type de tours de parole.................................................... 41

3.2.2 La longueur et la durée .................................................. 41

3.2.3 Les limites du tour de parole.............................................42

3.2.4 Les signaux de back-channel .............................................. 43

3.2.5 La délimitation des énoncés.............................................. 44

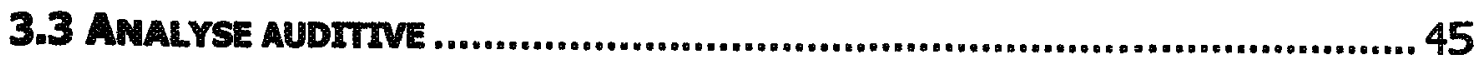

3.3.1 Les indices prosodiques................................................ 45

3.3.1.1 LES INDICES PROSODIQUES : LA PAUSE ET L'INTONATION .............. 46

3.3.2 Les indices paradiscursifs.............................................. 48

3.3.2.1 LES ÉLÉMENTS PHYSIOLOGIQUES ...................................... 48

3.3.2.2 LES HÉSITATIONS ......................................................... 48 
3.3.3 Les indices syntaxiques.................................................... 49

3.3.4 Les indlices sémantico-pragmatiques .................................... 50

3.3.4.1 LES IMDICES DE NON-CONCLUSION ....................................... 50

3.3.4.2 LES INDICES DE CONCLUSION ........................................... 50

3.3.4.3 LES INDICES DE JUGEMENT DE VALEUR ............................... 51

3.3.4.4 LES INDICES DE REDONDANCE......................................... 51

3.3.4.5 LA PRÉSENCE DE SIGNAUX EXPLICTTES DE FINALTÉ ET LES MOTS CLÉS.52

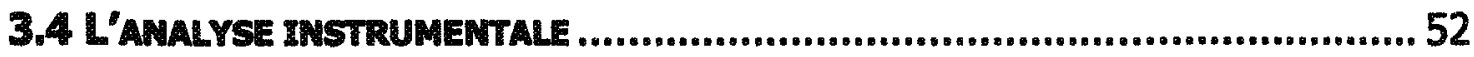

3.4.1 La numérisation ............................................................ 52

3.4.2 La segmentation ......................................................... 53

3.4.2.1 LA DÉ IMITATION DES PAUSES ........................................... 53

3.4.2.2 LES PROBLÈMES DE SEGMENTATION ...................................... 54

3.4.3 Les mesures de fréquence et d'intensité............................. 54

3.4.4 Les mesures de la durée ...................................................... 54

3.4.5 L'analyse des données..................................................... 55

3.4.5.1 LES MOYENNES .................................................................. 55

3.4.5.2 LA CHUTE DE FRÉQUENCE ET D'INTENSTTÉ .............................. 56

3.4.5.3 LES GRAPHIQUES ET LES TABLEAUX...................................5 56

CHAPITRE 4 : ANALYSE DES RÉSULTATS .............................................5 57

4.0 INTRODUCTION ......................................................................... 58

4.1 LES INDICES PROSODIQUES........................................................ 58

4.1.1 Les pauses ..................................................................... 58

4.1.1.1 LES TYPES DE PAUSES............................................... 59

4.1.2 L'intonation ...................................................................... 63

4.1.2.1 LES INDICES GLOBAUX ................................................. 63

4.1.2.2 LES INDICES INTERMÉDIAIRES OU SECTORIELS.......................... 68

4.1.2.3 LES INDICES LOCAUX................................................. 72 
4.1.2.3.1 La méthode de classement des schémas intonatifs. 72

4.1.2.3.1.1 Les neuf schémas intonatifs. 73

4.1.2.3.2 La comparaison en demi-tons de la fréquence des voyelles finales et pénultièmes............................. 77

4.1.2.4 SOMMAIRE DES OBSERVATIONS SUR L'INTONATION .......................78 78

4.1.3 L'intensité....................................................................... 79

4.1.3.1 LE TYPE D'ANALYSE....................................................... 79

4.1.3.2 L'INTENSTTÉ EN DISCOURS SPONTANÉ ...................................... 80

4.1.3.2.1 L'utllisation de lintensité dans le tour de parole...... 81

4.1.3.2.1.1 L'intensité moyenne ................................. 82

4.1.3.2.1.2 La ligne d'intensité globale....................... 83

4.1.3.2.1.3 L'intensité de la voyelle finale ................... 86

4.2 LES INDICES NON PROSODIQUES....................................................... 90

4.2.1 Les indices syntaxiques .................................................. 90

4.2.1.1 LA COMPLÉTION............................................................ 91

4.2.1.2 LA LONGUEUR ............................................................... 93

4.2.2 Les indices sémantico-pragmatiques ................................ 95

4.2.2.1 LA PRÉSENTATION DES INDICES ............................................96

4.2.2.1.1 Les indices de non-conclusion............................... 98

4.2.2.1.2 Les indices de conclusion...................................... 98

4.2.2.1.3 Les indices de jugement de valeur ........................ 99

4.2.2.1.4 Les indices de redondance................................... 100

4.2.2.1.5 Les signaux explicites de finalité ou mots clés....... 100

4.2.2.2 Recensement des indices sémantico-pragmatiques.......... 101

4.2.2.2.1 Les tours typiques et atypiques........................... 103

ANNEXE A : 


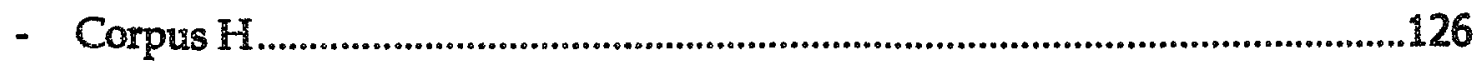

- Corpus F ........................................................................................................138

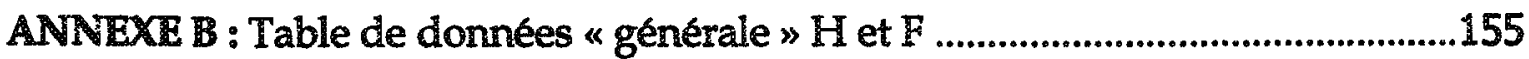

ANNEXE C : Table de données « intensite » $\mathrm{H}$ et $\mathrm{F}$......................................................161

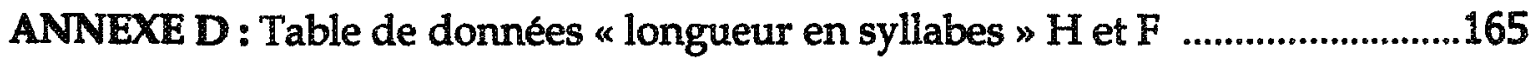

ANNEXE E : Tableau des indices de conclusion ....................................................169 


\section{LSTE DES GRAPHIQUE ET TABLEAUX}

Graphique :

Graphique 1: Déclinaison d'intensité des tours de parole de $H$ et $F$ avec valeur de pente $(m)$ et coefficient de détermination $\left(R^{2}\right)$. 84

Tableaux:

Tableau 1 : Durées moyennes des pauses (en cs) pour chaque locuteur selon le type de pauses incluant ou non les « zéros »

Tableau 2 : Comparaison de la valeur de Fo de la voyelle finale de tour par rapport à la moyenne de FO des voyelles cibles des énoncés du tour de parole .65

Tableau 3 : Comparaison de la valeur moyenne de FO des voyelles cibles de l'énoncé final par rapport à celle des autres énoncés du tour 66

Tableau 4 : Comparaison de la valeur de F0 (en $\mathrm{Hz}$ ) de la voyelle finale de tour par rapport à la moyenne de F0 des voyelles de fin d'énoncé d'un même tour de parole

Tableau 5 : La proportion de voyelles finales d'énoncé dont la fréquence est inférieure, supérleure ou égale à la moyenne de fréquence des voyelles cibles de l'énoncé correspondant (incluant l'énoncé final de tour).....69

Tableau 5.1 : La proportion de voyelles finales d'énoncé intratour dont la F0 est inférieure, supérieure ou égale à la moyenne des voyelles cibles des énoncés intratour correspondants

Tableau 5.2: Comparaison des F0 des voyelles finales de tour par rapport à la valeur moyenne des voyelles cibles de l'énoncé dont elle fait partie .71 
Tableau 6.1: Répartition des schémas intonatifs des voyelles antépénultièmes, pénultièmes et finales des énoncés finals de tour de parole dans les trois catégories .74

Tableau 6.2 : Répartition des schémas intonatifs des voyelles antépénultièmes, pénultièmes et finales des énoncés intratour dans les trois catégories.

Tableau 7 : Moyenne de variation intonative (en $\mathrm{Hz}$ et en \%) entre les voyelles pénultièmes et finales des tours à schéma intonatif descendant en comparalson avec la moyenne de variation intonative des voyelles pénultièmes et finales des énoncés intratour à schéma intonatif descendant.

Tableau 7.1: Valeur moyenne de variation en demi-tons des voyelles pénultièmes et finales d'énoncés intratour et d'énoncés finals de tour pour les énoncés descendants et pour les énoncés montants. .77

Tableau 8 : Moyenne d'intensité (en dB) de l'énoncé final (4 voyelles cibles) par rapport à la moyenne d'intensité des tours sans la moyenne de l'énoncé final ( 4 voyelles cibles par énoncé) 82

Tableau 9 : Valeur dintensité (en dB) de la voyelle finale de tour par rapport à la moyenne dintensité des voyelles cibles des tours sans la moyenne de l'énoncé final ( 4 voyelles cibles par énoncé)

Tableau 9.1: Comparaison des valeurs d'intensité (en $\mathrm{dB}$ ) de la voyelle finale de tour par rapport à la moyenne d'intensité des voyelles de fin d'énoncé d'un même tour de parole 
Tableau 10: Répartition des énoncés du corpus pour chacun des locuteurs par leur position dans le discours et selon quills sont déclaratif, interrogatif ou exclamatif

Tableau 11 : Longueur en syllabes orales des énoncés finals par rapport à la longueur moyenne en syllabes orales des énoncés intratour .94

Tableau 12 : Distribution et nombre d'utilisations des indices sémanticopragmatiques par catégorie dans les tours de parole pour les locuteurs $H$ et $F$ 102

Tableau 13 : Exemple de tour typique.................................................... 103

Tableau 14 : Exemple de tour atypique.................................................... 104 


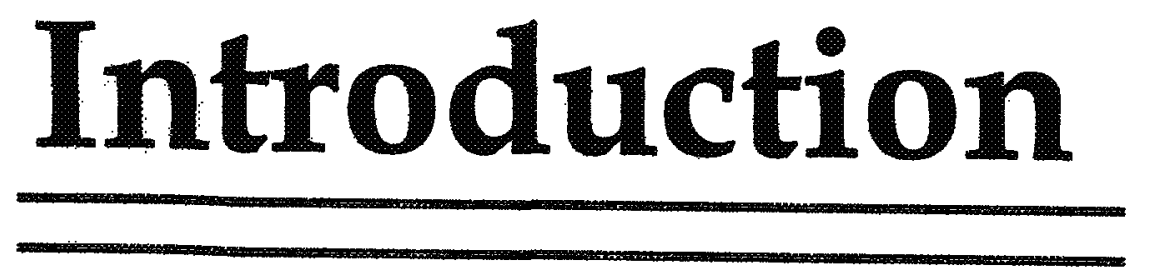


Depuis plusieurs années, la langue parlée a été étudiée par les linguistes et les phonéticiens sous divers points de vue et selon diverses méthodes. Si on a fait au départ un parallèle entre la langue écrite et la langue parlée, II ne fait cependant aucun doute maintenant que les deux types de discours ont des systèmes de fonctionnement somme toute assez indépendants. La syntaxe de l'oral, pour laquelle il est possible de revenir en arrière, ou encore de faire des répétitions sans brouiller le sens, n'est pas aussi rigide que la syntaxe de l'écrit, qui se doit de respecter un ordre strict et linéaire où il est impossible de revenir en arrière sans violer les règles de base (Ouellon, 1991). De même, un sous-entendu demande généralement à être suggéré à l'écrit d'une manière ou d'une autre, que ce soit par le choix des éléments lexicaux ou la construction syntaxique, alors qu'à l'oral, lintonation peut véhiculer une large part de la subjectivité sans que cette dimension ne soit explicitement verbalisée d'aucune façon.

Toute étude portant sur l'oral ainsi que son organisation se heurte au problème de la reconnaissance des unités constitutives (phonèmes, syllabes, groupes intonatifs, énoncés, tours de parole) et de leur organisation, de même qu'à celui des indices qui révèlent cette organisation. Ce problème est particulièrement complexe dans le cas des unités de plus grandes dimensions, soit l'énoncé et le tour de parole, ce dernier retenant ici notre attention. Il demande en outre à être abordé de manière différente selon que l'on a affaire à la lecture d'un texte ou à de l'oral spontané.

Une quantité assez importante d'études ont porté sur l'organisation prosodique du texte lu, parmi lesquelles celles de Lehiste et Peterson (1972) et Lehiste (1975). Avec ce type de discours, II est en effet plus facile d'éliminer linfluence des données trop disparates, d'avoir un corpus uniforme ou de contrôler les divers paramètres qu'on veut étudier. Les phrases sont aussi plus nettement découpées, ce qui fait que le locuteur sait plus facilement quand marquer la fin de celles-ci. Ce 
type d'études permet donc de bien isoler et cibler les divers paramètres en plus de bien en vérifier les impacts sur le langage.

Pius récemment, d'autres études se sont intéressées à la langue orale qui n'est pas soutenue par un texte préexistant dont elle constituerait en quelque sorte l'oralisation. Contrairement à ce qu'on peut penser, ce type de recherches ne porte cependant pas uniquement sur la parole spontanée. Les études sur le monologue en situation contrôlée, que l'on retrouve généralement sous forme d'instructions pour fabriquer quelque chose ou bien pour indiquer un itinéraire, semblent être plus nombreuses que les études qui portent sur le discours purement spontené. Swerts (1993, 1997 et 1998), Swerts et Geluykens (1994), Swerts et Collier (1992), Swerts, Collier et Terken (1994), Swerts et Ostendorf (1997) et Geluykens et Swerts (1993) sont les principaux représentants de ce type d'études sur le discours contrôlé, dont ils se sont attachés à faire ressortir les avantages, le principal étant la possibilité de profiter du meilleur des deux mondes ; il permet en effet de bien contrôler le contenu du corpus, qui se trouve du fait même partiellement homogénéisé, tout en laissant le locuteur libre de le dire comme il le désire. De cette façon, II est possible de comparer et d'évaluer des paramètres ciblés et leurs impacts sur le fonctionnement du discours, tout en préservant les qualités recherchées du discours spontané (voix naturelle, erreurs, hésitations, retours en arrière, répétitions, etc.), sans le côté artificiel du texte lu.

Les études sur l'organisation des unités de grandes dimensions dans le « vrai » discours spontané se font nettement plus rares, et ce, dans toutes les langues. En français québécois, les travaux de Ouellon (1990 et 1991), Ouellon, Paradis et Duchesne (1991) et Demers (1996) ont cependant abordé certains aspects de la question. Ce peu d'intérêt accordé à la forme de discours la plus représentative de l'usage naturel et quotidien du langage est probablement imputable à sa grande variabilité et à son caractère moins prévisible. En effet, lorsque le contenu du discours n'est pas prévu à l'avance, il est alors d'autant plus difficile de contrôler 
rigoureusement les paramètres à l'étude, ces demiers risquant toujours d'être infuencés de quelque manière que ce soit par le contexte du discours.

À cette imprévisibilitté inhérente au discours spontané, s'ajoute en ce qui concerne l'étude de l'organisation des tours de parole dans le dialogue, une seconde source d'imprévisibilité qui tient aux possibilités d'intervention de l'interiocuteur ; celui-ci peut en effet couper la parole au locuteur, refuser de la prendre lorsqu'on veut la lui céder, encourager le locuteur à continuer, réagir à ce qui est dit. Dans le cas du tour de parole long qui retiendra notre attention, le principal défi du locuteur dans ce type de discours est donc d'arriver à livrer son message et d'organiser son tour selon les différents topiques qu'll compte aborder, en signalant qu'il veut garder la parole ou au contraire la céder au moment opportun, tout cela en maintenant l'intérêt de son interlocuteur. Il s'agit là d'un processus relativement complexe, mais auquel chacun s'adonne à plusieurs reprises chaque jour de façon inconsciente dans le cadre des échanges sociaux. Il faut aussi considérer que linterlocuteur n'est pas passif pendant le discours du locuteur; il doit non seulement comprendre le message, mais aussi être attentif aux signaux de structuration du discours de façon à être prêt à prendre la parole à son tour lorsque cela lui sera demandé à travers le jeu dîndices plus ou moins explicites distillés par le locuteur.

Dans la présente recherche, nous avons voulu nous pencher sur ces divers indices ou signaux que donne le locuteur dans son discours pour indiquer qu'il veut céder la parole ou au contraire la conserver. Puisque notre étude sintéresse principalement à la dimension prosodique, notre attention s'est principalement portée sur les pauses, les différents indices intonatifs et les variations d'intensité. Par la suite, nous avons voulu explorer de façon complémentaire linfluence des autres facteurs linguistiques, tels la syntaxe, la sémantique et la pragmatique dans ce processus. 
L'étude porte sur un corpus de 2 conversations semi-dirigées, mais très naturelles, réalisées en laboratoire avec deux locuteurs qui se connaissaient bien. Nous avons retenu 20 tours de parole, c'est-à-dire 10 tours pour chacun des deux locuteurs, un homme et une femme étudiants universitaires dans la région de Québec. Les tours de parole, blen qu'appartenant à un sous-ensemble de tours longs, comprenant plusieurs énoncés, sont tous de longueurs différentes, et ils portent sur des thèmes variés. Les entrevues ont été réalisées quelques années avant le début de notre recherche et les locuteurs n'avaient donc aucune idée que leurs conversations seraient utilisées un jour pour une étude sur le tour de parole, ce qui exclut la possibilité d'effets intentionnels, comme c'est le cas dans certaines études portant sur les types de discours contraints mentionnées plus haut.

La présente recherche est divisée en 5 parties. Au chapitre 1, Problématique, nous commençons l'exploration de la question de recherche par une revue des connaissances générales sur les tactiques de changement de tour utilisées par les participants d'une conversation. Un rapide survol de quelques études sur le discours oral spontané est présenté avant de regarder de plus près le travail important de Duncan (1972) sur la conversation spontanée dans le but de préciser notre hypothèse de recherche.

Le chapitre 2, État de la question, propose un examen des diverses études qui abordent la question des tours de parole dans différents types de discours oraux. Une première partie consacrée aux indices prosodiques commence par un survol d'études importantes sur le discours lu auquel succèdent les études sur le discours spontané, le monologue et le dialogue. Dans la partie consacrée aux indices autres que prosodiques, nous abordons diverses études qui ont trait aux facteurs syntaxiques et sémantico-pragmatiques de l'analyse afin de mieux cerner ce dont il sera question dans l'Analyse des résultats. 
Dans le chapitre 3, Méthodologie, nous expliquons la procédure d'analyse que nous avons privilégiée pour mener à bien cette recherche. Elle se trouve divisée en 2 grandes sections, l'étude impressive, c'est-à-dire la méthode utilisée pour le prélèvement des indices à « l'oreille » et par la suite l'analyse instrumentale où l'on retrouve des indications sur le matériel informatique utilisé ainsi que sur la méthode de segmentation du signal acoustique et de prise de données.

Au chapitre 4, Analyse des résultats, les indices retenus sont traités de façon individuelle. On retrouve tout d'abord les résultats obtenus pour la pause, ensuite lintonation et enfin lintensité. Le rôle des facteurs linguistiques d'ordre syntaxique, sémantique ou pragmatique est brièvement exploré après celui des facteurs phonétiques. Dans ce chapitre, nous tentons d'évaluer l'apport de chacun des éléments dans l'annonce de la fin d'un tour de parole par le locuteur. Plusieurs tableaux synthèses nous permettent d'établir des valeurs moyennes et ainsi faire des liens, lorsque c'est possible, entre les divers éléments à l'étude.

Enfin, en Conclusion, on retrouve une récapitulation des principaux résultats obtenus. Nous avons aussi tenté de faire une synthèse de ce qu'ils nous révèlent sur le fonctionnement du tour de parole long dans un discours spontané de type collaboratif. 
Chapitre 1

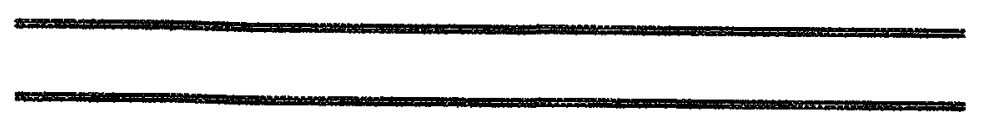

Problématique 


\subsection{INREDUCTION}

Dans son quotidlen, lêtre humain est appelé à communiquer avec les gens qui l'entourent et c'est généralement à l'aide de la conversation spontanée quill le fait, et ce, dans toutes les langues. Qu'il s'agisse dinterventions courtes ou longues, celles-ci ne sont généralement pas délimitées d'avance. On peut donc se demander comment font alors les participants à une conversation pour alterner leur tour à la parole sans empiéter sur le tour de leur interlocuteur. La présente recherche porte justement sur lidentification des mécanismes utilisés lors de la conversation pour marquer les limites des tours de parole longs.

Tout discours spontané implique énormément de facteurs qui influencent tous à leur manière la production du discours ainsi que son décodage. Il est d'autant plus difficile de contrôler les paramètres cibles alors que la quantité de facteurs impliqués est très importante, autant pour les facteurs prosodiques que syntaxiques et sémantico-pragmatiques. C'est la principale raison qui fait que la plupart des chercheurs préfèrent se pencher sur des discours plus prévisibles que la conversation spontanée, ce qui en limite ainsi le nombre de recherches qui sont consacrées à cette dernière. Il est toutefois nécessaire de faire une première tentative dans lidentification des éléments présents lors du processus d'alternance dans la conversation.

\subsection{LE TOUR DE PAROLE}

D'une manière générale, on sait que la conversation consiste en une alternance de tours de parole de longueurs et de durées diverses et que cette alternance est réalisée sans trop de difficulté par les locuteurs. Taylor et Cameron (1987) émettent deux hypothèses concernant la conversation : 
The first of theses assumptions is that conversations consist, at least in part, in the production of particular sorts of interactional units, such as speech acts, or moves, or turns, or sentences, etc. The second assumption is that in producing these units, conversationalists are guided by interactional rules wich determine when particular units may occur, how they may be recognized, what they may be combined with, and generally, how they fit into the organizational stucture of conversation. (Taylor et Cameron, $1987: 1$ )

Ces règles de l'interaction sont assez bien connues. On s'entend généralement pour affirmer qu'un tour de parole se termine le plus souvent avec le début du tour de parole suivant. On considère de la même façon que la fin d'une phrase, d'un paragraphe, d'un tour soit indiquée par différents indices tels une intonation descendante, un allongement des voyelles finales, des pauses plus longues, etc. Un des problèmes rencontrés en conversation est que tous ces éléments se retrouvent à lintérieur d'un même tour de parole. Le locuteur devrait alors marquer de façons distinctes la fin de ces différentes unités, c'est-à-dire marquer la fin d'un énoncé non final différemment de ce quill ferait pour un énoncé final s'il ne veut pas perdre son tour à la parole.

Geluykens et Swerts (1993) avancent que les locuteurs en situation de dialogue utiliseralent le ton haut pour les phrases non finales. Geluykens et Swerts (1994) et Swerts et Ostendorf (1997) ajoutent que le ton bas serait réservé pour marquer la fin d'un topique ou la fin d'un tour. Swerts, Collier et Terken (1994) rappellent que les syllabes en finale ont tendance à être allongées. Swerts, Wichmann et Beun (1996) et Swerts (1998) soulignent que la pause remplie est signe de continuité et que nous avons une tendance à associer une pause longue avec une frontière discursive importante. 


\subsection{UW TRAVAIL DE PRÉCURSEUR: : DUNCAN (1972)}

Dans sa recherche «Some signais and rules for taking speaking turns in conversations » qui se veut la base de plusieurs études sur le discours spontané et sur la conversation proprement dite, Duncan donne beaucoup de renseignements sur les signaux émis par le locuteur lorsquill veut céder le tour. L'étude est faite à partir d'un corpus constitué de deux entrevues réalisées entre un psychothérapeute et son client. Les entrevues ont été enregistrées sur ruban vidéo. Toutes les paroles des participants sont transcrites en plus de tous les mouvements produits par les locuteurs. Duncan a voulu noter le maximum d'informations puisquill cherchait à déterminer lequel des comportements observés est le principal élément qui influence une situation de communication; en plus des segments phonétiques, les éléments de paralangage et les mouvements du corps sont donc inclus dans la transcription du corpus.

Duncan identifle six indices « cède-tour » : lintonation, le paralangage (étirement exagéré de la syllabe finale ou de la voyelle accentuée du segment final), les mouvements du corps, les séquences sociocentriques (expressions stéréotypées du genre « mais euh» et «t'sais», le paralangage (chute de fréquence et/ou d'intensité «paralinguistiques» en conjonction avec une de ces séquences sociocentriques) et la syntaxe (complétion de la phrase). (p. 286-287)

L'analyse des entrevues montre que les locuteurs font preuve d'un grand respect de la prise du tour de parole. Les chances de tours simultanés diminuent quand l'interlocuteur essaie de prendre la parole après que le locuteur a émis des signaux de changements de tour. Plus Il y a d'indices de fin de tour, plus il y a de chances que l'interlocuteur tente de prendre la parole. Il n'y aurait pas d'élément spécifique en tant que tel qui influencerait cette situation de communication, mais plutôt des éléments plus généraux. À ce stade-ci, Duncan explique que les indices de fin qu'il 
identifie ne sont pas des indices émis expressément par le locuteur pour marquer la fin de son tour et que ce serait plutôt larrêt des éléments déjà en place qui annoncerait fa fin d'un tour. Il donne en exemple quil n'y a pas dintonation spécifique, mais une déviation du ton neutre. Il n'y a pas de mouvement corporel précis, mais l'arrêt du mouvement en court. Il n'y a pas non plus de schéma paralinguistique précis, mais plutôt une chute de fréquence et/ou d'intensité.

\subsection{VERS UNE HYPOTHESE}

L'étude de Duncan qui porte sur la conversation spontanée est très détaillée, mais elle est surtout axée sur son aspect social. Le point de vue phonétique y est moins développé. Par ailleurs, la plupart des études phonétiques sur le discours oral ne portent pas sur le discours spontané. Il est en effet plus fréquent de retrouver des études qui portent sur le discours lu (Lehiste, 1975) ou le monologue (Swerts, 1993, 1997, 1998; Swerts et Collier, 1992; Swerts, Collier et Terken, 1994, Swerts, Bouwhuis et Collier, 1994 ainsi que Swerts et Geluykens, 1994). Certaines études cependant vont porter sur le dialogue (Geluykens et Swerts, 1993 et 1994), mais en situation extrêmement contrôlée. Les acteurs de ces conversations sont souvent invités à indiquer la fin de leur tour par les movens phonétiques connus quîls croient appropriés. Les types d'études effectuées dans ces conditions sont nécessaires afin d'établir des bases de recherche solides à des études qui vont par la sulte porter sur des types de discours beaucoup moins prévisibles comme c'est le cas lic avec la conversation spontanée.

Notre recherche consistera ici à relever les données présentes à la fin des tours de parole d'une réelle conversation et non à produire artificiellement ce qu'on pense vouloir entendre à la fin des tours. En indiquant au locuteur quill doit utiliser des indices prosodiques pour signaler l'organisation de son discours, on risque de voir apparaittre ce que tout le monde croit devoir produire à la fin d'un énoncé et à plus 
forte raison à la fin d'un tour de parole. Le locuteur peut se sentir obligé de marquer ces finales avec une baisse d'énergie, une intonation descendante, un débit ralenti ou bien des pauses plus longues aux endroits désignés. C'est pour toutes ces raisons que le corpus choisi en est un qui a été produit par des locuteurs qui n'étaient pas informés que leur conversation servirait quelques années plus tard à une étude portant justement sur l'interaction. Nous avons alors une conversation réelle où les locuteurs n'utilisent pas de « recette » pour marquer la fin de leurs interventions. Nous nous retrouvons devant une conversation entre deux personnes où les locuteurs utilisent naturellement et inconsciemment des éléments à la fin de leur tour de parole pour ainsi indiquer à leur interlocuteur qu'ils ont fini de parler.

Nous tenterons alors de vérifier si ce qui est produit par les locuteurs, les éléments qu'on peut percevoir, laisse une trace au plan instrumental. De plus, pour ce qui est du contenu même du tour, nous devrons vérifier jusqu'à quel point les éléments syntaxiques et sémantico-pragmatiques influencent eux aussi l'alternance des tours de parole entre les locuteurs.

Dans Ihypothèse où les locuteurs qui participent à une conversation annoncent la fin de leur tour de parole, queis moyens linguistiques utilisent-ils? Trois grandes familles de facteurs linguistiques sont ciblées: les facteurs prosodiques, les facteurs syntaxiques et les facteurs sémantico-pragmatiques. Pour chacune de ces trois familles de facteurs, plusieurs indices peuvent être identifiés. Pour les facteurs prosodiques, nous nous intéresserons à la pause, à lintonation et aux variations d'intensité; parmi les facteurs syntaxiques, nous retiendrons le type de phrases, la longueur des énoncés et la complétion grammaticale; enfin, plusieurs indices de conclusion et de non-conclusion seront pris en compte au plan sémanticopragmatique. Parmi ces éléments, lesquels sont précisément utilisés? Dans quelle mesure sont-ils employés? Dans le cas où plusieurs éléments sont utilisés à la fin d'un même tour, y a-t-il un élément plus important que les autres? 
Même sil n'est pas évident d'analyser avec précision ces indices dans un discours aussi variable et imprévisible que la conversation spontanée, il est pratiquement impossible de ne pas détecter la présence d'au moins quelques-uns de ces indices à la fin des tours. Notre recherche servira de champ exploratoire à ce domaine de langue parlée afin de faire linventaire de ce qui se retrouve à la fin d'un tour de parole long, en conversation spontanée, en français québécois. À la lumière de nos résultats, nous espérons que d'autres chercheurs seront tentés de pousser plus loin l'étude prosodique des changements de tour de parole. 


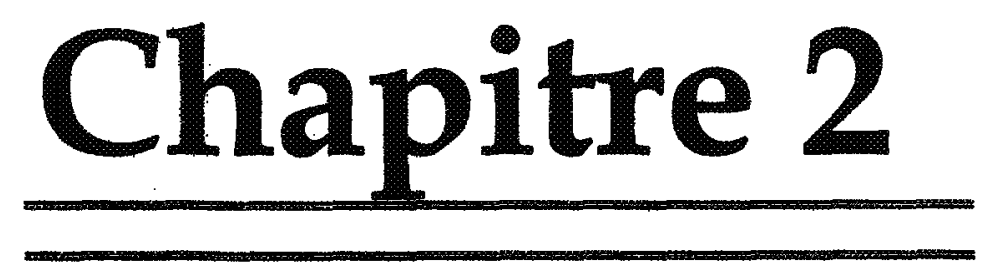

État de la question 


\subsection{IRTRODUCTION}

Comme l'étude prosodique de la conversation orale spontanée semble représenter une problématique récente, les recherches reliées à ce sujet sont peu nombreuses. Par contre, l'analyse prosodique du discours, au sens large, a été effectuée sur plusieurs plans. Des travaux portant sur les aspects prosodiques de divers types de discours, l'énoncé, le « paragraphe », le monologue et la conversation spontanée, ont alors été consultés pour cet état de la question. Cette étude sur les facteurs prosodiques des changements de tour de parole doit se faire en tenant compte également des autres facteurs qui peuvent intervenir dans la perception d'une fin de tour. Les facteurs syntaxiques et sémantico-pragmatiques n'ont pas été oubliés. La mise en parallèle des résultats des différentes recherches permettra d'obtenir un certain portrait des éléments étudiés dans le discours oral, portrait qui servira d'assise à l'analyse des résultats.

\subsection{Le TRAVAIL DE PRÉCURSEUR DE LEHISTE: DU disCOURS LU AU SPONTANÉ}

Les études prosodiques sur le discours lu sont plus anciennes et sont en quelque sorte à la base des études sur le discours spontané. Le caractère plus structuré, plus fixe, du discours lu offre en effet une certaine sécurité quant à l'analyse et à la validité des résultats obtenus. Ce type de discours permet également moins de possibilités de retour en arrière ou de faire des répétitions ou des erreurs puisqu'il présente des contraintes à la souplesse à lintérieur desquelles ॥ est impossible d'improviser : le lecteur doit s'en tenir au texte quill lit. C'est ce qui explique qu'on a d'abord vu apparaître des études sur les unités du discours lu comme le texte ou le «paragraphe ${ }^{1} »$. On ne doit alors pas s'étonner que ces études aient servi

\footnotetext{
${ }^{1}$ Ce n'est évidemment que par analogie que l'on peut parler de « paragraphe » dans le discours oral spontané. Lehiste et Wang (1977 : 278), dans les instructions données au sujet, caractérisent cette unité en ces termes : « A paragraph boundary indicates the end of a fairly complete part of the narration and the beginning of a new thought,
} 
également de départ aux études prosodiques du discours plus spontané qui sont apparues par la suite.

Lintérêt qu'on porte ici aux particularités du discours lu, principalement le " paragraphe », vient du fait que, à plusieurs reprises, les chercheurs soulignent les similarités importantes entre les unités de discours plus grandes (topique, tour de parole). Les deux unités ont un début, un corps ou un développement et une conclusion. Il est donc à l'avantage de notre recherche de prendre à tout le moins connaissance de certaines études phonétiques sur le « paragraphe » lu afin d’avoir quelques points de repère pour orienter notre recherche.

Ilse Lehiste est l'une des premières à s'intéresser à la structure prosodique globale du discours spontané, spécialement dans une perspective de rapprochement avec la structure du paragraphe. Dans son étude préliminaire The phonetic structure of paragraphs (1975) où elle effectue des tests de perception afin de déterminer l'emplacement de phrases en contexte de «paragraphe » par opposition aux mêmes phrases dites de manière isolée, elle avance I'hypothèse que le «paragraphe » pourrait posséder une structure suprasegmentale qui en indiquerait le début et la fin et qui en même temps caractériserait le corps du « paragraphe ». Cela implique que le contour intonatif d'une phrase produite à lintérieur d'un « paragraphe », qu'elle soit au début, au milieu ou à la fin, serait différent de celui de la même phrase dite en contexte isolé. En d'autres mots, Lehiste pense quill est possible que le «paragraphe » soit caractérisé par une structure intonative globale à laquelle le contour intonatif des phrases le constituant serait en quelque sorte subordonné. Il en irait de même pour le rythme et l'accentuation. Pour vérifier cette hypothèse, elle a constitué un corpus à partir de 3 phrases pouvant être facilement permutées sans poser de problèmes de compréhension. Ces phrases ont été alors toutes prononcées en contexte isolé d'une part et également en contexte de «paragraphe» d'autre part (6 possibilités). Elles ont ensuite été extraites de leur contexte avant d'être soumises 
à des sujets afin que ceux-ci puissent identifier les phrases comme étant une phrase en contexte isolé ou bien une phrase en contexte de « paragraphe " (tâche A); les sujets devalent ensuite dire si cette phrase se trouvait, selon eux, en position initiale, milieu ou finale de « paragraphe » (tâche B).

Parmi les différences qui ressortent, sur le plan acoustique, Lehiste relève le fait que les phrases isolées ont une durée plus longue que leur phrase correspondante en contexte de «paragraphe ». De plus, les phrases ayant une F0 plus élevée ont eu tendance à être identifiées à une phrase produite en tête de «paragraphe » (tâche B). Enfin, pour les 3 énoncés à l'étude, la FO au début de la phrase initiale de « paragraphe » avait une valeur plus élevée que celle de la phrase en contexte isolé. Elle avance donc la possibilité que le locuteur commence à une fréquence plus élevée dans le but de se donner assez de hauteur pour produire une descente intonative sans descendre au niveau de la laryngalisation (creaky voice) au beau milieu du « paragraphe », ou sans avoir à revenir à une fréquence plus élevée au milieu du « paragraphe » ce qui pourrait être interprété comme de l'emphase par linterlocuteur.

Lehiste vérifie alors I'hypothèse qu'il serait possible pour l'auditeur d'identifier qu'une phrase est produite de façon isolée ou qu'elle est produite à l'intérieur d'un « paragraphe ». D'un point de vue perceptif, elle remarque que les phrases les mieux reconnues sont les phrases produites isolément, ce qui s'expliquerait, selon elle, par les différences de longueurs entre les deux types de phrases à l'étude (tâche A). De façon assez surprenante, ce sont les phrases de début de « paragraphe » qui prêtent le plus à confusion quant à leur identification par les auditeurs en dépit du fait que ces phrases présentent une FO plus élevée. Lehiste propose l'explication que le contour intonatif final de «paragraphe» a une fonction grammaticale particulière et que c'est pour cette raison que les énoncés finals sont mieux perçus. 
Dans une étude ultérieure de 1977, Perception of boundaries with and without semantic information Lehiste et Wang font une incursion directe du côté du discours spontané dans le but de vérifier lapport de la dimension phonétque dans la reconnaissance des limites de phrases et de «paragraphes» en discours spontané. À laide de tests de perception portant sur un discours spontané d'environ une minute, présenté à 24 sujets auditeurs, dans une version filtrée (inintelligible) et une version normale, Lehiste et Wang tentent de déterminer si les auditeurs peuvent percevoir et distinguer les fins de phrases et les fins de « paragraphes » et cherchent à identifier les indices utilisés pour ce faire.

Les résultats sont sensiblement différents selon que l'on a affaire à la version filtrée (donc inintelligible) ou à la version normale. Globalement, les sujets ont identifié beaucoup plus de limites de phrases ou de «paragraphes » dans la version filtrée, pour laquelle ils ne disposaient que des indices prosodiques, que dans la version normale pour laquelle ils pouvaient exploiter aussi des indices syntaxiques et sémantiques. Le temps de réaction étant par contre beaucoup plus important pour la version normale, les sujets se donnaient l'opportunité d'exploiter tous les indices possibles.

S'il y a, dans plusieurs cas, convergence entre les réponses des sujets pour la version filtrée et normale, les cas de divergence sont cependant nombreux. Puisqu'lls ne disposent que des indices suprasegmentaux, les sujets identifient plusieurs limites quils ne retiennent plus lorsquils disposent de l'ensemble des informations syntaxiques et sémantiques contenues dans la version normale; le nombre de ces « fausses identifications » non confirmées est de l'ordre de $40 \%$.

Lehiste et Wang disent finalement que les sujets semblaient pouvoir distinguer assez nettement les limites de « paragraphes » de celles de phrases même dans la version filtrée. Ils s'avouent cependant impuissants à identifier les indices 
phonétiques utilisés pour ce faire même s'il semble que des facteurs comme l'abaissement de fréquence et la laryngalisation de même que la durée de la pause et l'allongement prépausal pourraient jouer un rôle à cet effet.

Dans une étude subséquente, Lehiste (1979) cherche précisément à vérifier dans quelle mesure cette capacité à distinguer prosodiquement les limites de phrases des limites d'unités plus grandes, que sont les «paragraphes», pourrait effectivement reposer sur les différents degrés d'allongement des segments qui précèdent la pause marquant la fin de la phrase ou du paragraphe. La procédure utilisée est assez semblable à celle utilisée dans Lehiste et Wang (1977), les sujets devant indiquer les fins de phrases et les fins de «paragraphes» en écoutant successivement la version filtrée et la version normale de l'enregistrement d'une entrevue semi-dirigée. Les résultats discutés sont ceux obtenus avec la version filtrée, c'est-à-dire celle où les seuls indices disponibles pour opérer la distinction sont d'ordre phonétique, à l'exclusion de toute information syntaxique ou sémantique. L'analyse des résultats amène à rejeter l'hypothèse initiale, l'allongement ne pouvant être retenu comme le facteur pouvant rendre compte de la capacité des sujets à faire la distinction entre les deux types de limites. Lehiste suggère que la reconnaissance des fins de paragraphes repose en fait sur une interaction dans des proportions variables entre trois facteurs que sont une plus grande longueur de la pause, la présence de laryngalisation en finale et l'allongement prépausal, sans qu'aucun ne soit obligatoire ni, à lui seul, déterminant.

\subsection{LES TRAVAUX PLUS RÉCENTS SUR LE SPONTANÉ}

Suite aux travaux de Lehiste effectués principalement sur le discours lu, des études portant sur des discours dits spontanés sont apparues, certains se rapprochant plus ou moins de l'usage réel du langage. Même si on les qualifie de spontanés, les 
corpus utilisés étalent souvent très contrôlés et parfois très artificiels. Les auteurs de ces études; qui ont toutes pour objectif de mieux comprendre l'utilisation et forganisation de la prosodie dans ce type de discours, justifient cette rigidité par un important besoin de précision dans lidentification des facteurs prosodiques qui jouent un rôle dans l'organisation du type de discours ciblés.

\subsubsection{Les indices phonétiques : les travaux de Swerts}

Parmi les chercheurs les plus présents dans ce domaine, on retrouve notamment Marc Swerts et toute l'équipe de chercheurs de l'Institute for Perception Research (IPO) à Eindhoven (Pays-Bas). Les champs d'intérêt explorés étant en général assez près de notre problématique, on trouvera là une source d'information très importante pour notre recherche.

Swerts s'est intéressé au discours oral spontané sous plusieurs facettes. Au départ, on parle surtout de travaux portant sur l'étude de discours spontanés, plus ou moins naturels, allant du monologue jusqu'à la conversation, tantôt contrôlés, tantôt libres. Plus récemment, il n'est pas rare de voir son nom associé à des recherches se concentrant principalement sur la synthèse de la parole et plus spécifiquement sur la prosodie de ce type de discours afin de rendre plus naturel et plus fluide linteraction homme-machine.

\subsubsection{LES INDICES PHONÉTLUES DANS LE MONOLOGUE}

À travers ses nombreuses recherches, Swerts s'est beaucoup intéressé au monologue dit de description (Swerts ,1993, 1997, 1998; Swerts et Collier, 1992; Swerts, Collier et Terken, 1994; Swerts, Bouwhuis et Collier, 1994 et Swerts et Geluykens, 1994). Les locuteurs devaient alors donner des indications (itinéraire, instructions d'assemblage ou descriptions de suites de formes géométriques et 
d'ceivres d'art) de façon spontanée pour des ins d'enregistrement en suivant un schéma. À travers ce type de discours, Swerts et l'équipe de l'Institute for Perception Research cherchalent à analyser des facteurs bien précis, dans un contexte tout aussi précis, dans le but d'évaluer l'importance du rôle des facteurs prosodiques dans l'organisation du discours.

Pour ce faire, les éléments non prosodiques du discours ont dû être neutralisés le plus possible afin de minimiser leur influence au plan de la perception de l'organisation du discours à l'étude. Tout en émettant un discours dit « spontané », les locuteurs devaient strictement respecter un contenu prédéterminé. Les tests de perception ont porté sur le rôle des éléments prosodiques à caractère intonatif (le contour intonatif, le registre et l'étendue de fréquence), sur des indices temporels (durée du mot final, longueur des pauses, débit) et sur la distribution des pauses (silencieuses ou remplies). L'intensité n'a en général pas été évaluée dans les études mentionnées ici. Les chercheurs n'écartent tout de même pas qu'elle puisse jouer un rôle dans la perception de l'organisation du monologue (Swerts et Geluykens, 1994 : 26).

Les études de Swerts et al., qui portaient sur le monologue, cherchaient à comprendre l'organisation de linformation dans le discours (topiques) ou bien la perception de la finalité à différents degrés d'importance et comportaient généralement des tests de perception. Les auditeurs devaient alors se prononcer sur limportance de la pause entendue (changement de phrase, de topique ou une fin de monologue) ou sur la place relative des phrases dans le monologue (début, milieu, fin).

Swerts et al. font alors ressortir plusieurs éléments de ces études. Dans un premier temps, nous nous attarderons aux études portant sur la perception de la finalité. 
Piusieurs aspects de la prosodie ont été considérés à travers ces recherches, dont, au premier titre, tout ce qui touche à la fréquence.

\subsection{Le contour intonatif des monologues d'instructions}

Nous nous intéressons d'abord à ce que ces études peuvent nous apprendre sur le contour intonatif d'un monologue. Il semble en effet que les locuteurs étudiés avaient tendance à produire des schémas intonatifs particuliers à des endroits bien précis dans leurs discours (Swerts, Collier et Terken, 1994 : 85) et que certains contours semblent mieux concorder avec un énoncé de fin que d'autres (Swerts, Bouwhuis et Collier, 1994: 2073). On retrouverait plus fréquemment une intonation montante dans une position non finale d'énoncé. En finale, on peut retrouver deux types de courbes intonatives, une première qui descend pour ensuite remonter légèrement et une deuxième qui présente une courbe descendante. Cette dernière est utilisée également en finale de description (ou de monologue) (Swerts et Collier, 1992 : 466). La mélodie des énoncés joue donc un rôle dans l'identification de la finalité (Swerts, Bouwhuis et Collier, $1994: 2073$ ), ces schémas intonatifs étant perçus par les auditeurs.

Du point de vue acoustique, Swerts, Collier et Terken (1994) nous signalent aussi que les valeurs de FO finale de la dernière phrase d'un monologue seraient généralement plus basses que les valeurs de FO des phrases internes. Il y aurait également quelque chose de particulier avec l'avant-dernière phrase d'un monologue. En effet, celle-ci aurait des valeurs de fo finale plus basses que les phrases non finales, quoique moins basses que la dernière phrase. Les chercheurs concluent que les phrases précédant l'avant-dernière semblent donner des indications prosodiques claires de non-finalité, c'est-à-dire un contour intonatif montant avec un allongement prononcé du dernier mot du segment. Dans l'avantdernière phrase, le locuteur utiliserait plus souvent des éléments prosodiques quil 
sont généralement associés à la finalité, comme un contour intonatif relativement bas qui se terminerait plus près de la base du registre du locuteur, ainsi qu'une absence de l'aliongement du mot final. Du point de vue de l'audlteur, ce changement de comportement de la part du locuteur à partir de l'avant-dernière phrase annoncerait que la fin du discours approche (Swerts, Collier et Terken, $1994: 88)$.

Cette observation avait déjà été faite dans une étude de Swerts en 1993 où if constatait que les sujets étaient capables dídentifier l'avant-dernière phrase d'un monologue et que l'identification de la dernière phrase en était d'autant plus facile. Il observait alors aussi une forte tendance, même si elle n'était pas significative d'un point de vue statistique, à la descente de la FO finale et à une prononciation de plus en plus rapide du mot final à partir de l'avant-dernière phrase.

Ceci nous amène donc à la question du débit et de l'allongement du dernier segment des énoncés. Dans Swerts, Collier et Terken (1994), l'analyse auditive suggère que les mots qui se retrouvent en fin d'énoncé subissent un allongement dans toutes les situations sauf dans la phrase pénultième. Cet allongement est en quelque sorte supprimé, donnant alors limpression que la pénultième forme un tout avec la phrase finale (Swerts, Collier et Terken, $1994: 85$ ). Il y aurait alors une augmentation du débit sur les deux dernières phrases d'un monologue, la pénultième ne présentant aucun allongement, laissant ainsi entendre à l'auditeur que la fin approche. À la lumière des tests de perception effectués, une phrase entendue serait classée plus près de la fin d'un monologue si elle présente une F0 basse et un mot final prononcé plus rapidement.

Si on regarde maintenant la variation du registre moyen, on note qu'il tend à descendre en suivant la progression d'un monologue (Swerts et Collier, 1992). Les phrases de début afficheraient des fréquences hautes, celles du milieu auraient des 
valeurs intermédiaires et les phrases situées à la fin seraient plus basses. Cette tendance est confirmée dans Swerts (1997). En ce qui concerne l'étendue du registre (mesurée par l'écart type en deml-tons), les phrases de début présenteraient l'étendue la plus large et les phrases finales la plus étroite, les phrases internes ayant alors une étendue se situant entre les deux autres (Swerts et Collier, 1992 : 467).

\subsection{L'organisation en topiques}

L'organisation des unités d'information contenues dans le discours, nommées topiques, a fait l'objet d'études dans Swerts et Geluykens (1994), Swerts (1997) et Swerts (1998). Comme il est mentionné dans Swerts et Geluykens (1994), les locuteurs utilisent plusieurs moyens non prosodiques pour indiquer qu'une section de discours constitue une unité de topique. Mais, ces éléments non prosodiques sont le plus souvent renforcés par des éléments prosodiques afin de structurer plus clairement le discours.

Les variations de fréquence qui constituent le contour intonatif sont un de ces indices prosodiques structurants et on observe en effet que celles-ci ne sont pas aléatoires. Étudiant des monologues de consignes ou dindications, Swerts et Geluykens (1994) ont pu confirmer qu'un contour final bas coincide avec la fin d'une instruction et inversement qu'un contour final haut coïncide avec la nonfinalité d'une instruction. Ils expliquent alors que la fonction des contours bas situés aux fins des instructions serait de signaler qu'une unité informationnelle a été complétée. La fonction des contours non bas qui surviennent généralement à IIntérieur des unités serait de vouloir dire qu'il reste encore des choses à dire sur le même sujet (Swerts et Geluykens, 1994 : 27). Cependant, ils nous font remarquer que : « [...] while a high-ending contour almost invariably signals that an instruction has not ended, it is not the case that a low-ending one automatically 
implies instruction-finality 》 (Swerts et Geluykens, 1994 : 28). Cela nous rappelle que les contours intonatifs descendants se retrouvent également à des endroits qui ne sont pas finals et qu'ils ne sont maiheureusement pas exclusifs aux énoncés de fin de discours ou de fin dinstruction.

Swerts et Geluykens (1994) suggèrent également de porter une attention particulière aux indices globaux de fréquence, ce qu'on peut appeler aussi la ligne de déclinaison. Selon eux, le locuteur ferait une gestion globale de la fréquence tout au long de son discours. Ils expliquent que la Fo serait en moyenne plus haute au début d'une unité pour descendre doucement tout au long de cette unité et remonter à nouveau au début d'un nouveau topique (Swerts et Geluykens, 1994 : 33). De façon similaire, dans un autre type d'étude, Swerts et al. (1996) ont remarqué une relation entre la valeur de la remontée de fréquence et limportance de la pause. Une pause située entre deux énoncés s'accompagnerait généralement d'une remontée de fréquence plus importante qu'une pause entre deux syntagmes.

Dans Swerts (1997 : 520), des observations préliminaires auraient aussi permis d'observer que les transitions entre les unités informationnelles majeures (les topiques) sont souvent accompagnées par des expressions d'hésitation comme les pauses remplies. Ces pauses remplies, se retrouvant à des ruptures importantes du discours, tendent à être différentes des autres aux plans segmental et prosodique. De plus, elles sont plus souvent précédées et suivies par des silences (Swerts, 1997 : 520; 1998 : 494). Ce type de pauses remplies pourrait aussi jouer un rôle dans l'organisation en topiques du discours. Dans Swerts, Wichmann et Beun (1996) et dans Swerts (1998) on s'est effectivement aperçu que les ruptures importantes du flot informatif sont plus sujettes à être accompagnées de pauses remplies que les ruptures moins importantes. Ces pauses apparaissent très tôt dans la phrase et présentent souvent une fréquence plus haute et une durée plus longue que les pauses remplies qui surviennent ailleurs dans le discours (Swerts, 
Wichmann et Beun, $1996: 1035)$. Les chercheurs avancent alors lhypothèse que cette pause remplie, produite en position initiale de topique, a pour fonction de faire comprendre à linterlocuteur quili est en train de structurer le discours quill va produire. De là lidée que la pause remplie est un marqueur organisationnel du discours en opposition aux rires, à la toux ou aux grognements qui n'en sont pas. Il semblerait qu'en néerlandais, ces pauses remplies soient considérées comme des marqueurs discursifs à part entière parce qu'elles fonctionnent de la même manière que les autres marqueurs (Swerts, 1998 : 494).

Pour ce qui est des pauses silencieuses, que nous appellerons simplement pauses, il faut garder en tête qu'elles ne surviennent pas uniquement aux frontières syntaxiques d'énoncé. De la même manière, les frontières syntaxiques d'énoncé ne sont pas toutes accompagnées de pauses (Swerts et Geluykens, 1994 : 34). On peut cependant percevoir une tendance à associer une pause longue avec une frontière importante : plus la frontière discursive est importante, plus la pause tend à être longue. Dans le même ordre didée que Swerts et Geluykens (1994), ten Bosch, Oostdijk et Boves (2005) mentionnent que les tours de parole ne sont pas toujours nettement délimités par une pause et qu'il arrive même régulièrement qu'une juxtaposition entre les tours des locuteurs se produise, entrainant l'absence totale de pause.

La durée des pauses pourrait alors être en relation avec la structure en topiques du discours. Swerts et Geluykens (1994: 33), observent en effet que les pauses les plus longues se retrouvent entre les instructions; les pauses qui suivent l'énoncé introducteur d'un nouveau topique sont en effet habituellement plus longues que les pauses situées ailleurs dans le topique. Swerts (1997) avance également lídée que la durée des pauses diminue, en même temps que le débit augmente, au fur et à mesure de la baisse du degré d'incertitude du contenu du discours. Plus le discours progresse vers sa fin, plus le locuteur parlera vite et plus ses pauses seront courtes. 


\subsubsection{LES INDICES PHONÉTIQUES DANS LE DIALOGUE}

Lintérêt de Swerts pour le discours spontané ne s'est pas limité à lětude du monologue. En effet, parallèlement à ses travaux sur la perception de la finalité et lorganisation en topiques dans le monologue spontané, Swerts et les autres chercheurs affiliés au groupe de recherche de l'Institute for Perception Research ont également porté leur attention sur le dialogue (Geluykens et Swerts, 1993 et 1994) et, plus récemment, sur le dialogue homme-machine (Swerts et Ostendorf, 1997; Krahmer, Swerts, Theune et Weegels, 2002 et Hirschberg, Litman et Swerts, 2004). Bien que nous soyons consciente que beaucoup de facteurs prosodiques peuvent être employés de la même manière dans les deux situations langagières (monologue et dialogue), le but ici est de mettre en lumière les éléments qui pourraient être plus particuliers au dialogue.

Le premier défi d'un locuteur qui détient la parole dans un dialogue est d'arriver à bien faire comprendre son message sans se faire interrompre. Pour y arriver, on peut supposer que le locuteur utilise une stratégie prosodique particulière qui est différente de la stratégie du monologue. Les résultats des recherches de 1993 et 1994 de Geluykens et de Swerts tendent à confirmer cette hypothèse, du moins dans le type de discours particulier quîls ont étudié; il s'agit en effet d'une situation expérimentale fortement contrainte impliquant la description d'une série de figures géométriques (les séries), présentées en groupes de figures liées entre elles (les suites de figures), chaque série pouvant comprendre un nombre variable de groupes présentant un nombre variable de figures de forme et de couleur différentes. Trois conditions particulières ont été testées selon que la consigne donnée au sujet était : a) de faire ressortir l'organisation en suites, ou b) de signaler explicitement à son interlocuteur la fin d'une série pour que celul-ci puisse prendre la parole afin d'amorcer une description à son tour, ou encore c) de marquer à la fois les fins de suites et la fin de série. On aura compris que le premier cas, qui insiste sur l'organisation de l'information en topiques, s'apparente 
plus au monologue descriptif alors que les deux autres constituent nettement des situations de dialogue, le locuteur se concentrant sur la seule dimension interactonnelle dans le deuxième cas (dialogue simple) alors quil doit gérer simultanément la dimension informationnelle et la dimension interactionnelle dans le troisième (dialogue complexe). Il ressort de l'analyse acoustique des séquences produites que les fins de séries sont systématiquement marquées par un ton bas en situation de monologue comme dans les deux situations de dialogue. La situation est cependant différente pour les fins de suites. En effet, si les locuteurs, en situation de dialogue complexe, ont utilisé presque exclusivement des tons hauts à la fin des suites qui ne sont pas des fins de série, on observe par contre une répartition égale entre tons hauts et tons bas en situation de monologue. Cette nette préférence pour les tons hauts en situation de dialogue pourrait s'expliquer, selon Geluykens et Swerts (1993), par le fait que le locuteur sait qu'il risque d'être interrompu par son partenaire sill utilise un ton bas à cet endroit. Il réserverait donc le ton bas pour les fins de groupe qui constituent en même temps la fin de la série, et donc la fin du tour de parole (Geluykens et Swerts, 1994).

Il ressort donc des études de Geluykens et Swerts (1993; 1994) que les locuteurs utillsent effectivement des indices prosodiques locaux, en l'occurrence une distribution différente de contours intonatifs finals, pour marquer à la fois la structure de linformation (organisation en topiques ou en groupes) et la gestion de linteraction (en marquant les fins de séries qui correspondent à des changements de tour). Mais un des apports importants de cette étude est de montrer que les locuteurs seraient en outre capables d'annoncer en quelque sorte à l'avance le mouvement intonatif final à travers les fréquences finales des segments internes de groupe; c'est ainsi que les voyelles finales des contours internes situés à l'intérieur d'une suite se terminant sur un ton haut sont sensiblement plus hautes que la fréquence moyenne du locuteur et inversement pour les contours situés à lintérieur d'une suite se terminant par un ton bas. $\grave{A}$ un plan plus global, la fréquence finale de n'importe quel contour intonatif serait alors 
un bon indicateur de son appartenance à une suite se terminant sur un ton haut ou un ton bas et donc en définitive de sa position dans le discours (Geluykens et Swerts, 1993; 1994).

Encore plus significatif est le fait que les auditeurs sont sensibles à la présence de tels indices, du moins dans la situation expérimentale fortement contrainte en cause. Dans un deuxième volet de leur étude, Geluykens et Swerts se sont attardés à vérifier si les récepteurs peuvent effectivement utiliser /information prosodique disponible pour juger si tel ou tel segment présenté se situait en début, en millieu ou en fin de discours. Il ressort que les sujets peuvent assez facilement distinguer le caractère final ou non final d'un extrait non seulement sur la base de I'orientation du contour intonatif final, mais aussi, en l'absence d'un tel contour final, en exploitant le caractère plus ou moins haut ou bas des contours intonatifs internes. Si le caractère très concluant des résultats ne laisse aucun doute sur l'utilité des indices prosodiques pour la gestion de linteraction dans la situation expérimentale en cause, les chercheurs ne se prononcent cependant pas sur la généralisation de la conclusion à des situations de discours plus naturelles où plusieurs autres facteurs entrent en jeu (Geluykens et Swerts, 1994).

En ce qui concerne la pause, on voit dans Swerts et Ostendorf (1997:33) que les phrases initiales d'un dialogue homme-machine ont généralement un plus grand nombre de pauses que les autres phrases de ce type de dialogues. Ce nombre est peut-être en relation avec un plus grand nombre de mots dans la phrase initiale, mais cela rappelle tout de même ce qui a été observé précédemment pour le monologue; le début était en effet marqué par un plus grand nombre d'hésitations et de pauses remplies (Swerts, 1997 et Swerts, Collier et Terken, 1994), reflet d'un plus important travail de planification (Swerts, Wichmann et Beun, 1996 : 1035). Par contre, Swerts et Ostendorf n'ont noté aucune différence au plan du débit, alors que dans le monologue, le locuteur montrait une tendance à parler plus vite 
à mesure que la fin du monologue approche (Swerts et Ostendorf, 1997 : 32). Ils ne sont cependant pas en mesure de préciser s'il s'agit là d'une caractéristique générale du dialogue ou plutôt d'un effet spécifique du contexte homme-machine.

\subsubsection{Les indices non prosodiques}

Même si les éléments prosodiques de la conversation retiennent plus particulièrement notre attention dans cette recherche, nous ne pouvons pas ignorer la présence ni limportance des éléments non prosodiques de la conversation. En effet, selon Geluykens et Swerts (1994), l'auditeur serait capable d'extraire de linformation au plan de l'organisation informationnelle et interactionnelle à partir des indices non prosodiques, textuels et contextuels. Nous porterons alors notre attention du côté des éléments syntaxiques et sémanticopragmatiques qui peuvent être présents dans la réalisation d'un discours spontané.

\subsubsection{LES FACTEURS SYNTAXIQUES}

Au fil du temps, les linguistes qui ont étudié la langue orale se sont aperçus qu'un phénomène très intéressant se produisait lors de l'émission d'un discours oral spontané. En effet, ces chercheurs se sont rendu compte que la syntaxe utilisée lors d'une production orale était très différente de la syntaxe utilisée lors d'une production écrite. Cette différence peut être perçue de plusieurs façons. Fontaney (1987 : 225) nous fait part d'une réaction qu'ont certaines gens lorsqu'ils se rendent compte de cette différence : "Quand nous lisons la transcription d'une conversation authentique ou d'un dialogue spontané, la pauvreté du langage, lîncohérence de la syntaxe, le manque de structure logique nous frappent, voire nous scandalisent. » Ouellon (1991) fait lui aussi un parallèle entre la syntaxe de l'écrit et la syntaxe de i'oral : 
À l'écrit, l'unité de discours se définit relativement blen. Elle est normalement marquée par des ponctuations fortes. Elle se distingue aussi par une structure syntaxique complète et une certaine unité sémantique. Ces caractéristiques ne valent cependant plus en discours oral, spontané de surcroît. L'unité syntaxique peut être rompue, l'unité sémantique peut très bien ne pas exister. L'oral spontané n'est pas exempt de reprises, d'hésitations, de pauses imprévues... (p. 63).

De son côté, Rémi-Giraud (1987) explique que l'échange dans une conversation est l'équivalent de la phrase dans un texte parce qu'ils sont tous les deux précédés et suivis, au plan phonétique, d'une pause forte qui coincide avec une pause de la voix. Molbaek Hansen, Reinholt Peterson et Spang-Hanssen (1993) font également part de leurs observations concernant le rapport pause et frontière syntaxique. Ils ont constaté que la majorité des pauses de leur corpus surviennent à une frontière syntaxique et qu'elles auraient tendance à survenir vers la fin d'une phrase plutôt qu'au début. On retrouve dans l'étude de Bestgen (1998) des affirmations qui vont dans le même sens que Molbaek Hansen et al. (1993).

Pour ce qui est du rôle de la syntaxe à la fin des tours de parole, Cutler et Pearson (1986) soutiennent que celui-ci est d'une grande importance, sans oublier celui de la sémantique et de la référence au contexte. Ils expliquent que pour prendre son tour de parole au bon moment, \|l est préférable de déceler rapidement que le locuteur finit ou va finir son tour. Pour ce faire, Cutler et Pearson soutiennent que l'on s'appuie sur un certain nombre de connaissances implicites comme le fait qu'un énoncé repose habituellement sur une structure syntaxique complète, sur le fait qu'une question demande habituellement une réponse ou encore sur le fait que les anecdotes ont une fin (même s'il n'y a pas toujours de dénouement ou de chute). Ils nuancent cependant la portion de Duncan qui inclut dans ses six signes d'un changement de tour, la syntaxe qu'il définit comme «la complétion grammaticale d'une proposition, impliquant une combinaison sujet-prédicat » (p. 286-287). Selon Cutler et Pearson, la complétion syntaxique d'une proposition ne saurait à elle seule constituer un indice de changement de tour très fiable 
puisque si c'était le cas, I'nterlocuteur tenterait de prendre le tour de parole à chaque fin de phrase.

Le type de phrases rencontrées dans les diverses études sur le dialogue ou le discours spontané n'est pas très souvent mentionné. Lors quill est mentionné, c'est parce que la phrase déclarative est elle-même à l'étude. Tout de même, le type de phrases qui seraient le plus généralement utilisées pour communiquer une information, ou énoncer un fait serait le type déclaratif selon Dolbec et Ouellon (1987). Il semble en effet raisonnable de penser que puisqu'un locuteur prend généralement la parole pour transmettre un message, son tour de parole sera caractérisé par une majorité de phrases déclaratives, la phrase interrogative étant, dans la majorité des cas, un indice explicite que le locuteur souhaite passer la parole à son interlocuteur.

Dans un autre ordre diidée, la longueur des phrases ou des énoncés pourrait jouer un rôle dans l'annonce de la fin d'un tour de parole. Swerts et Ostendorf (1997), comme nous le mentionnions plus haut, ont remarqué que les locuteurs avaient tendance à faire des phrases plus longues en début de tour, ce qui laisse supposer que les phrases en finales pourraient être plus courtes puisque, toujours selon Swerts et Ostendorf, les informations redondantes sont alors plus facilement omises.

\subsubsection{LES FACTEURS SÉMANTICO-PRAGMATIQUES}

Il est intéressant de s'attarder aussi à la façon dont le locuteur laisse transparaître ses intentions dans son discours. Les facteurs que nous qualifions de sémanticopragmatiques nous aident en ce sens. Ces facteurs ne se présentent pas nécessairement sous une forme lexicale précise dans le discours. Ils peuvent prendre plusieurs formes et répondent également à plusieurs questionnements. 
Molbaek Hansen et al. (1993) laissent sous-entendre que la phrase aurait non seulement une structure grammaticale propre à elle-même, mais aussi une structure liée à la transmission du message.

Bestgen (1998) pense lui aussi que la phrase a une structure liée à la transmission du message. Le locuteur utiliserait des marqueurs de segmentation du genre du « et » pour ainsi mettre en évidence les changements de topique ou ses intentions à garder la parole. Il cite en exemple le « et» émis pendant une pause. Sa fonction est alors de remplir la pause afin dinformer linterlocuteur que le discours va continuer tôt ou tard. Dans le même ordre d'idée, Fontaney (1987) nous parle de « line-holding » qui sont des mots utilisés en conjonction avec lintonation montante pour signifier que le locuteur cherche ses mots [ « euh », « je ne sais pas moi»]. Dans le présent travail, ces éléments seront placés sous l'étiquette d'indices de non-conclusion.

Les indices de conclusion peuvent prendre plusieurs formes eux aussi et ne sont pas si évidents à identifier. Ils sont généralement très liés au discours. Les mêmes mots peuvent prendre différents sens selon ce qui est dit par le locuteur. Selon Cutler et Pearson (1986), les interlocuteurs savent que les anecdotes racontées ont nécessairement des fins, ce qui laisse entendre que l'on peut prévoir la fin d'un tour lorsque le locuteur approche de la conclusion de son récit.

Dans son étude sur les récits d'expériences personnelles, Labov (1978 : 306-307) illustre la structure générale d'un récit. Cette structure comprend 6 parties :
1. Résumé : de quol s'agit-il?
2. Indications : quil, quand, quoi, où?
3. Développement : et après, qu'est-ce qui s'est passé?
4. Evaluation : et alors?
5. Résultat ou conclusion : comment cela s'est-ill fini?
6. Chute : ne répond à aucune question 
Dans cette structure, nous nous intéressons plus aux parties 5 et 6 , c'est-à-dire le résultat ou la conclusion et la chute. Selon Labov, la conclusion « marque le terme de la série d’événements que décrit le récit» (p. 298). Cette «conclusion» justement semble effectivement correspondre à ce que nous avons nommé indices de conclusion.

Pour ce qui est de la chute, c'est dans cette partie que le locuteur annonce la fin de son récit. Elle peut également lui servir d'emplacement pour \& faire quelques observations d'ordre général ou à expliquer l'effet qu'ont produit sur lui les événements » (Labov, 1978 : 301). Les éléments que représente cette chute semblent correspondre à ce que nous avions nommé « indices de jugements de valeur» dans une étude préliminaire (Lepage, 1996), nom que nous avons conservé pour ce travail. Ces éléments peuvent prendre plusieurs formes, comme on peut le voir dans les deux exemples suivants, provenant de deux récits différents (Labov, 1978 : 301):

Récit 1:

« Ouais, c'te bagarre, c'a été une des plus importantes. »

Récit 2:

« On m'a donné le reste de la journée. »

« Et depuis, le mec, je l'ai plus revu pa'ce que maintenant je laisse tomber, je laisse tomber, vous voyez. "

« Plus de problèmes. " 
Cutler et Pearson (1986) ainsi que Fontaney (1987) nous rappellent quant à eux que les fins de tour ne sont pas toujours annoncées très longtemps à l'avance. On peut avoir-affaire-ici à des signaux-explicites de finalité. Un locuteur peut simplement poser une question, alors linterlocuteur sait automatiquement que c'est à son tour de parler. Cutler et Pearson affirment que les questions obligeant une réponse, II serait alors impoli de ne pas le faire. Sous un autre angle, fontaney souligne, elle aussi, que les questions demandent des réponses et cela même si leur contenu n'est pas conclusif comme peuvent l'être celui des énoncés déclaratifs et même si elles sont généralement accompagnées d'une intonation montante, laquelle est plutôt associée à la non-finalité. Cela veut donc dire que la finalité peut être annoncée peu de temps à l'avance par des indices très ponctuels comme c'est le cas avec les questions.

Dans notre étude préliminaire sur les changements de tour de parole (Lepage, 1996), d'autres indices ponctuels ont aussi été relevés et regroupés sous l'étiquette de « mot clé ». Ces indices sont des mots qui ont le sens de « j'ai fini de parler et tu peux prendre la parole maintenant » et la forme la plus courante dans nos corpus est le mot « finalement ». 
Tran:

ines

... Sauf que quand j'ai traversé l'boulevard, j'suis venu pour arrêter. Puis malheureusement l'en..., par rétropédalage ça freinait pas. Alors, j'suis passé tout droit. Pis y avait une Camaro, qui devait rouler à quatre-vingts kilomètres heure...

Ouf!

... qui m'a poignée. Fait que le bicycle était fini. Moi, j'al fait quelques pieds dans les airs. Pis, j'avais juste quelques égratignures, finalement.

I Eh! Ton heure était pas arrivée to:.

Ces mots clés ne fonctionnent pas exactement comme les indices de conclusion en général puisquill ne s'agit que d'un mot et non d'un énoncé ni d'un segment d'énoncé, comme on peut le voir dans l'exemple qui précède. 


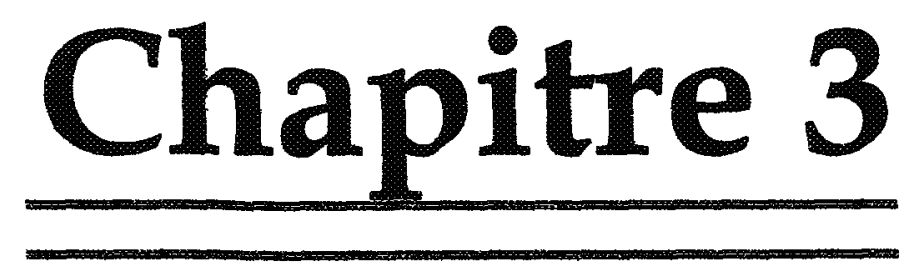

Méthodologie 


\subsection{INTRODUCTION}

Pour mener à blen cette recherche, nous avons choisi une méthode d'analyse qui S'inspire et s'apparente à celle utilisée par Touati $(1991,1993,1995)$ ainsi que Bruce et Touati (1992) dans la perspective où une analyse en plusieurs volets correspondait à notre objectif initial. La méthodologie quilis proposent est divisée en 4 parties (analyse discursive, analyse auditive, analyse acoustico-phonétique et analyse par synthèse). L'étude des tours de parole en français québécols n'étant qu'ầ ses premiers balbutiements, l'analyse par synthèse a donc été écartée ici.

Notre recherche propose donc une analyse auditive, qui vient compléter une analyse sémantico-pragmatique et une analyse phonétique, qui se veut plutôt prosodique, le tout portant sur un corpus de conversations. Nous verrons, dans les pages qui suivent, une présentation détaillée du corpus et de sa constitution ainsi qu'une explication plus détaillée de ce que nous entendons par ces trois analyses.

\subsection{Présentation du Corpus}

Le corpus sélectionné a déjà été utilisé par d'autres chercheurs faisant partie du projet PROSO. Il est constitué de quatre entrevues qui mettent chacune en présence deux locuteurs. Ce corpus présente plusieurs caractéristiques intéressantes pour l'étude des tours de parole et se prête bien au type de recherche que nous voulons effectuer.

Les entrevues retenues sont, par ailleurs, d'une bonne qualité sonore puisqu'elles ont été enregistrées en laboratoire. Elles se prêtent donc bien à l'analyse instrumentale, ce qui augmente l'intérêt d'étudier ce corpus. Il s'agit de conversations semi-dirigées mettant en présence un intervieweur et un interviewé. Ce type de conversations implique que les locuteurs savent implicitement quilis ont 
chacun un rôle à jouer. Le rôle de lintervieweur est de faire parler le plus possible le locuteur et celui du locuteur est de parler le plus possible. Nous avons tout de même choist d'étudier ce corpus pulsque les tocuteüurs présents lors des conversations se connaissent bien et sont assez famillers. Ce qui devait être au départ une entrevue semi-dirigée prend plutôt la forme d'une conversation de type amicale et spontanée.

Notre corpus se distingue donc de ceux de la plupart des études précédentes par son caractère « naturel ». À la différence de la majorité de ces dernières, il ne s'agit pas d'oralisation d'un texte écrit préexistant, ni de production d'un discours fortement contraint par le contenu informationnel sous-jacent ou la nature de la tâche, comme c'est le cas par exemple pour les discours de description ou dinstructions. C'est donc pour cette première raison que l'on peut parler de discours spontané. On peut en outre considérer que la relation de familiarité qui existe entre les participants et la façon dont se déroule la conversation permettent d'employer le qualificatif spontané dans un deuxlème sens, soit celui que lui donne Labov (1976 : 146) comme le style de parole qui, à l'intérieur du discours formel créé par la situation d'entrevue, se retrouve lorsque les participants portent le moins d'attention à leur langage et s'approchent ainsi du parler ordinaire.

\subsubsection{Choix du corpus}

Les quatre entrevues du corpus de départ ont été réalisées avec la participation de quatre locuteurs : deux hommes ( $H$ et $H 1$ ) et deux femmes ( $F$ et $F 1$ ). F agit comme intervieweuse dans trois entrevues ( $F$ et $F 1, F$ et $H, F$ et $\mathrm{H1}$ ). Dans la quatrième entrevue, $H$ prend le rôle d'intervieweur et $F$ devient linterviewée (entrevue $H$ et $F$ ). Les quatre locuteurs sont des Québécois de niveau universitaire ágés de 20 à 30 ans. Deux entrevues ont été retenues pour constituer ce corpus, $F$ et $\mathrm{H}, \mathrm{H}$ et $\mathrm{F}$. Les deux autres présentaient des éléments peu favorables à une 
étude précise sur les facteurs prosodiques présents à la fin des tours de parole comme des éclats de rire trop fréquents en fin d'énoncé ou de tour ou bien un locuteur peu loquace es qui ne parle pas assez fort pour instrumentation.

\subsubsection{Constitution d'un corpus}

À partir de ces deux conversations, un corpus de tours de parole longs a été constitué. Pourquoi faire une telle sélection? Les conversations retenues pour constituer le corpus offraient toutes une grande diversité de tours de parole pour ce qui est des tours longs et des tours plus courts. Une recherche sur les tours de parole longs a été préférée parce que, tout d'abord, une étude sur les tours de parole en général est beaucoup trop vaste. Cela nous aurait donné des résultats peu significatifs à cause de la très grande variété de tours. C'est ainsi que les tours de parole longs, souvent de type narratif, donnent lieu à une organisation du discours de la part du locuteur. Les tours de parole courts, quant à eux, se présentent fréquemment sous la forme de question et réponse ce qui fait que leur structure est très différente.

Pour constituer un corpus de tours de parole longs acceptable, vingt tours ont été sélectionnés, dix tours pour chaque locuteur. Ce nombre de tours de parole est moins important que le nombre initialement prévu avant que le rejet de deux entrevues nous amène à une révision à la baisse. De plus, même si les conversations offraient un assez grand choix de tours de parole longs, ceux-ci n'étaient pas tous du même type. Nous avons cherché à retenir des tours de parole longs qui avaient une certaine ressemblance et une certaine homogénéité, ce qui a également contribué à faire baisser le nombre de tours de parole disponibles. Quelques critères de sélection ont été établis afin doobtenir cette homogénéité. 


\subsection{SÉRECTON DES TOURS DE PAROLE LONGS}

\subsection{Le type de tours de parole}

Le premier critère de sélection était de déterminer le type de tours de parole long auquel nous allions nous intéresser. Nous avons décidé de nous concentrer sur le récit des événements de la vie quotidienne, ce que Labov (1978) appelle des « récits d'expériences personnelles ${ }^{2}$, ce qui veut dire que nous avons exclu les tours de parole qui étaient des explications techniques (explication du protocole d'enregistrement) ou des argumentations très marquées. Les récits d'expériences personnelles sont des histoires qui impliquent le locuteur quil les raconte. On peut y retrouver des événements de la vie quotidienne tout comme des sujets de l'actualité. Les locuteurs partagent leurs opinions sur certains sujets tout en écartant les argumentations trop marquées.

\subsubsection{La longueur et la durée}

Pour des raisons pratiques, la première sélection des tours de parole longs s'est faite à partir de la transcription des enregistrements des deux entrevues retenues. Pour en déterminer la longueur, nous avons compté le nombre de lignes de chacun des tours. Un minimum de trois lignes pour les tours de parole longs a été fixé comme seull acceptable, toutes les interventions plus courtes étaient considérées comme des tours de parole courts.

2 Ce choix s'est imposé de lui-même d'autant plus facilement que les « récits d'expériences personnelles » représentaient de loin la forme la plus fréquente dans le corpus de tours sélectionnés. 
Même si nous nous intéressons principalement aux tours de parole longs, la durée précise du tour en secondes n'étalt pas un critère déterminant au départ étant donné que la première- sélection s'est faite à partir de la transcription. Au cours de l'analyse instrumentale, la durée des tours de parole longs sera notée puisqu'elle peut parfois nous venir en aide dans l'analyse de l'ensemble des tours de notre corpus.

\subsubsection{Les limites du tour de parole}

Dans un premier temps, les limites des tours de parole ont été acceptées telles qu'elles apparaissaient dans la transcription. Par la suite, les limites de tours identifiées ont été affinées par l'écoute minutieuse de l'enregistrement de chacune des conversations. Par cette démarche nous voulions identifier avec le plus de précision possible le début et la fin des tours de parole retenus. Nous nous sommes assurée que linterlocuteur (intervieweur), lorsqu'il parlait au début du tour, avait vraiment la parole, c'est-à-dire qu'il ne s'agissait pas ici d'un signal de back-channel, tel que nommé par Yngve (1970). Une fois cette étape réalisée, nous nous assurions que le locuteur poursuivait sur le même sujet pendant au moins quelques lignes (min. 3 lignes).

Pour identifier la fin du tour, lintervention de linterlocuteur servait encore une fois de limite. Cette intervention se devait de constituer un tour de parole en sol. Même si la plupart d'entre elles ne posaient pas de réels problèmes, il était cependant parfols moins évident d'identifier certaines interventions de linterlocuteur pour savoir sil s'agissait bien d'un nouveau tour de parole ou, au contraire, d'un simple signal de back-channel. Ces signaux de back-channel étaient d'ailleurs le principal obstacle rencontré au moment de la délimitation des tours de parole longs. 


\subsubsection{Les signaux de back-channel}

La notion de signal de back-channel est très ambigüe et les chercheurs ne s'entendent pas pour ce qui est d'y voir un changement de tour de parole ou non. Yngve (1970) et Fries (1952) considèrent que le back-channel ne sert pas à demander la parole ni à interrompre le tour de parole en cours, mais plutôt à indiquer que le message passe bien. Laforest (1992) demeure somme toute assez prudente quant à la définition qu'elle donne des signaux de back-channel. Selon elle, certains signaux sont considérés comme étant des changements de tour de parole (métaquestion, reformulation, etc.) tandis que d'autres sont uniquement * de brèves contributions de lauditeur ${ }^{3}$ qui semblent le ponctuer [le discours du locuteur] » (Laforest, 1992 : 31).

Les signaux de back-channel ont été considérés comme des signaux qui confirment la réception d'un message émis et il n'y a alors pas de changement de tour aux endroits où les signaux de back-channel sont présents. Cette décision est fondée sur les propos de Laforest qui nous dit que, en somme, les signaux de backchannel qui peuvent être des tours de parole veulent généralement dire que Iinterlocuteur prend la parole pour dire qu'il veut que le locuteur poursuive son tour (Laforest, 1992 : 192). Étant donné quilis sont perçus en tant que façon de confirmer celui qui parle dans son rôle de locuteur, nous les avons alors considérés comme faisant partie des événements habituels survenant lors des tours de parole longs.

Par ailleurs, les tours sélectionnés se terminaient en général au moment où linterlocuteur émettait une contribution assez longue, c'est-à-dire un énoncé de plusieurs mots, ce quil permettait d'éviter toute ambiguité. Nous avons constaté à la lecture du corpus que ces contributions représentaient habituellement une

\footnotetext{
${ }^{3}$ Les brèves contributions en cause correspondent à : hum hum, oui/non, OK, etc.
} 
rupture dans le discours du locuteur. En effet, les tours de parole longs sont le plus souvent produits sur un même topique, un même thème, un sujet unique du début à la fin du tour, et ce, pour chaque tour. Dans ces conditions, Imitervention de linterlocuteur sur un aspect nouveau du même topique ou, à plus forte raison, sur un autre topique sert de limite (Lepage, 2000). Cette façon de faire permet d'écarter les interventions qui sont des signaux de back-channel qui visent plutôt à assurer la continuité du discours du locuteur même si la distinction n'est pas toujours facile à faire en pratique.

\subsubsection{La délimitation des énoncés}

Une première délimitation des énoncés a été faite avec l'aide de la transcription du corpus, mais en gardant en tête que nous avions affaire à un discours oral spontané. Il est important ici de souligner que la syntaxe de l'oral est très différente de celle de l'écrit et que l'énoncé au sens où nous l'entendons rejoint un peu plus lidée de « utterance » que celle de phrase en français. Crystal (1985: 322) caractérise « utterance» en tant qu'unité physique (par opposition à la phrase qui est une unité grammaticale) correspondant à toute séquence de parole [stretch of speech] précédée et suivie par un silence ou un changement de locuteur, encore quili reconnaît que cette définition n'est pas pleinement opératoire. On ne peut en effet identifier un énoncé seulement en disant qu'il représente ce qui est prononcé entre deux pauses silencieuses ou bien qu'il soit terminé par un changement de locuteur. Il arrive souvent que deux énoncés se succèdent sans quill y ait de pause ni de changement de locuteur. La délimitation des énoncés de notre corpus s'est donc faite de façon empirique, et pour ainsi dire mixte, en associant les critères de complétude syntaxique et/ou sémantique aux critères purement physiques que constituent les indices prosodiques. Nous avons ensuite procédé à une seconde écoute de la cassette audio pour vérifier cette première délimitation et faire, sill y a lieu, les ajustements nécessaires afin 
d'obtenir la délimitation la plus plausible possible. Un énoncé peut alors être très long ou bien très court, tout dépend de la complexité de lidée véhiculée par le locuteur. Nous navons pas ixe de longueur minimale ni maximale. Nous avons alors obtenu 74 énoncés pour le locuteur $H$ et 77 pour le locuteur $F$, dont 10 énoncés finals pour chacun des locuteurs.

\subsection{Analyse auditrive}

L'analyse du corpus se divise en deux grandes étapes: l'analyse auditive et l'analyse instrumentale. La première étape consistait à faire de façon auditive le relevé des différents indices présents dans les tours de parole. Le relevé porte sur les indices présents à la fin des énoncés comme à la fin des tours de parole pour identifier ceux qui peuvent être considérés comme caractéristiques de la fin des tours de parole. La présence de ces indices a été notée à partir de l'enregistrement et de la transcription des conversations. Ces indices peuvent être de divers ordres : prosodique, paradiscursif, syntaxique, sémantico-pragmatique.

\subsubsection{Les indices prosodiques}

La prosodie concerne généralement « les éléments dynamiques de la chaîne parlée » comme les « variations de hauteur, dîntensité, de durée, qui déterminent la mélodie, les tons, les pauses, les accents et le mthme et qui sont intégrés globalement au niveau perceptif par lintonation. Ces éléments sont aussi appelés suprasegmentaux» (Landercy et Renard, 1977 : T10). Évidemment, l'étude de tous ces éléments en même temps apparaît très difficile, voire même impossible à réaliser. C'est pourquoi, à la lumière des travaux antérieurs, nous nous concentrons principalement sur quelques aspects, dont lintonation (montante, descendante et neutre), la pause (courte ou longue, remplie ou silencieuse) ainsi que lintensité. 
Puisque cette recherche porte sur la conversation, il semble important que les éléments étuciés solent repérables par ceux qui participent à la conversation. Nous avons choisi de demeurer assez près de ce que fon entend lors d'une conversation en retenant dans un premier temps des éléments identifiables à l'oreille, éléments que nous cherchons ensuite à quantifier par le biais d'une analyse instrumentale. Afin de produire cette analyse instrumentale, il fallait d'abord identifier les éléments cibles dans les tours de parole du corpus.

\subsubsection{LES INDICES PROSODIQUES : LA PAUSE ET L'INTONATION}

Lors d'une première analyse auditive, les indices prosodiques reliés à la pause et à Iintonation, ont été relevés à lintérieur des tours de parole longs pour les opposer aux indices prosodiques présents à la fin des tours de parole longs, dans le but d'en dégager des différences permettant d'identifier les indices de fin de tour de parole. Dans le cas de lintensité, plus difficile à décrire avec précision, on s'est contenté à ce stade, de commentaires du genre: «le locuteur parle plus fort, parle moins fort, etc. », l'analyse de ce paramètre devant surtout se faire dans le cadre de l'analyse instrumentale.

La pause silencieuse et la pause accompagnée d'un signal de la voix (remplie) peuvent avoir des fonctions différentes. Même si la pause est généralement accompagnée des effets de lintonation, elle joue tout de même un rôle très important sur la perception de la fin d'un tour de parole, qu'elle soit silencieuse ou remplie. D'après Rémi-Giraud (1987), la pause silencieuse serait un indice de finalité. Dans un échange entre deux locuteurs par exemple, la pause fait comprendre à l'interlocuteur que le locuteur lui laisse le tour de parole, d'où sa signification de finalité. 
Contrairement à la pause silencieuse, la pause remplie aurait comme fonction de signiffer à linterlocuteur que le locuteur veut conserver la parole ou qu'll souhaite simplement reformuler son idée (Matasci-Galazzi et Pedoya-Guimbretière, 1987). La pause remplie peut également jouer un rôle dans l'organisation du discours, comme nous l'avons vu précédemment avec Swerts, Wichmann et Beun (1996) et Swerts (1998) à la partie 2.2.1.1.2 L'organisation en topiques. Les pauses remplies font partie de la grande famille des hésitations. Cependant, elles sont traitées séparément puisqu'elles peuvent entraîner des phénomènes différents au plan conversationnel (voir la partie 3.3.2.2 Les hésitations).

En ce qui concerne Iintonation, élément qui occupe en général une place privilégiée dans l'étude du discours, nous avons dû faire preuve de réalisme et de discernement. Le mouvement intonatif étant présent partout dans toute production orale, nous avons concentré notre étude sur les variations intonatives qui précèdent les pauses longues ou courtes. L'étude approfondie de l'ensemble de la courbe intonative aurait exigé un travail trop important, et qui n'était pas absolument indispensable, compte tenu de notre objectif qui est d'identifier les indices pouvant influencer la perception de la fin d'un tour de parole. Nous avons donc choisl de porter notre attention sur les moments privilégiés où il peut y avoir changements de tour afin de repérer la présence d'indices intonatifs que Iinteriocuteur pourrait interpréter en ce sens.

À partir de la transcription du corpus et de l'enregistrement sur bande magnétique, nous avons donc relevé ponctuellement, toujours «à l'oreille», les variations intonatives perceptibles au moment où le locuteur produisait une pause à lintérieur ainsi qu'a la fin du tour de parole. Cette vérification auditive des variations intonatives est très importante ici, car nous cherchons à reconnaitre les éléments, présents dans une conversation et perçus par l'interlocuteur, qui 
régulent en quelque sorte les changements de tour de parole et à savoir si l'élément intonation fait partie de ces régulateurs.

\subsubsection{Les indices paradiscursifs}

Les indices paradiscursifs sont aussi d'une grande importance. Effectivement, en analyse du discours, ces indices cachent beaucoup d'intentions de la part du locuteur qui sont difficiles à cerner, mais parfois ils peuvent aider à comprendre la structure du tour de parole. Plusieurs indices contextuels ou paradiscursifs (resplration, déglutition, bruit de fond, etc.) ont été notés, puisque ceux-ci semblent souvent entrainer la présence de phénomènes prosodiques comme des variations d'intonation et des pauses de diverses longueurs.

\subsubsection{Les ÉLÉMENTS PHYSIOLOGIQUES}

La respiration et la déglutition sont des perturbations physiologiques très importantes qui surviennent lors de la production du discours oral. Ces événements ont été notés, parce qu'ils s'accompagnent souvent de phénomènes prosodiques comme des pauses de diverses longueurs et des variations d'intonation.

\subsubsection{LES HÉSTTATIONS}

Les hésitations provoquent généralement des phénomènes prosodiques différents de ceux que la respiration ou la déglutition peut amener. L'hésitation prend le plus souvent la forme de la répétition d'un même son, comme «à, à, à... », d'une erreur de prononciation « ... y nous $a, y$ nous ra, y nous a ramené », mais elle peut aussi se manifester par une pause remplie (généralement un [ө] allongé) qui est plutôt utilisée pour signifier son désir de conserver la parole comme nous l'avons 
vu au point 3.3.1.1 Les indices prosodiques: la pause et /intonation. Inversement, Thésitation peut amener une coupure de parole de la part de linterlocuteur. Il arrive en effet fréquemment que, lorsque le locuteur hésite dans ses mots, linterlocuteur lui coupe la parole pour l'aider à retrouver ses idées. Cela se traduit, sur le plan prosodique, par une absence de pause à la fin du tour de parole. Cette intervention de linterlocuteur en situation d'hésitation du locuteur peut même entrainer, au plan conversationnel, un changement forcé de tour, avant que le locuteur n'ait terminé son propos.

\subsubsection{Les indices syntaxiques}

L'étude des indices syntaxiques s'est principalement faite par le biais de lidentification des types de phrases présentes dans les tours de parole, la longueur en syllabe des énoncés et la complétion grammaticale. Une notation simple et assez sommaire du genre phrase «déclarative », « interrogative $»_{\text {g }}$ « exclamative» est utilisée. Cette notation nous permet de tracer un portrait général des types de phrases utilisées par les locuteurs pendant la production de leurs tours de parole. À partir de là, on vérifiera la possibilité que le type de phrases utilisées dans le tour de parole puisse s'accompagner d'un schéma intonatif particulier pouvant aider le locuteur à signifier quîl souhaite céder la parole, notamment dans le cas de phrases interrogatives.

On a aussi noté dans quelle mesure les énoncés en cause pouvaient être considérés comme «complets» sur le plan grammatical. Duncan (1972: 287) identifiait la complétion grammaticale comme une marque de finalité. Enfin, le nombre de syllabes phonétiques par énoncé a été calculé afin de voir sill existait une différence de longueur entre les énoncés finals et non finals. 


\subsubsection{Les indices sémantico-pragmatiques}

Des indices sémantico-pragmatiques, dans le tour de parole long, peuvent aussi laisser entendre à l'interlocuteur que le locuteur n'a pas fini de parler, est sur le point de finir ou a fini de parler. Ces indices sont en principe très liés au contenu du tour lui-même. Nous les avons répartis dans cinq classes, c'est-à-dire les indices de non-conclusion, les indices de conclusion, les indices de jugements de valeur, les indices de redondance et les signaux explicites de finalité et les mots clés.

\subsubsection{LES INDICES DE NON-CONCLUSION}

Les indices de non-condusion, tout comme leur nom l'indique, sont caractérisés par des mots ou expressions qui laissent sous-entendre que le locuteur poursuivra son tour de parole. En réalité, les indices de non-conclusion sont présents lorsque le locuteur annonce de façon plus ou moins explicite qu'il va poursuivre ce qu'il raconte déjà. Par exemple dans Lepage (1996), quand le locuteur dit : « ...j'étais sous la douche, pis jime suis dit: ... » (tour 3), ce qui est souligné est un bon exemple d'indice de non-conclusion puisque linterlocuteur sait qu'il doit attendre pour savoir ce que le locuteur s'est dit. Par contre, d'autres indices de nonconciusion sont beaucoup moins faciles à cerner que celui du tour 3 , comme : * ...Fait qu'à un moment donné... » (tour 12). II est bien certain que ces mots laissent limpression que le locuteur n'a pas fini son tour sauf que ces expressions sont peut-être moins explicites que celles du tour 3.

\subsubsection{LES INDICES DE CONCLUSION}

Les indices de conclusion sont essentiellement caractérisés par des termes qui dévoilent la conclusion. Le dévoilement de la conclusion apparaît en quelque sorte lorsque le locuteur raconte la fin de son récit, lorsqu'il en donne l'élément clé. Par 
exemple, si le locuteur parie d'une promesse tout le long de son récit, la fin surviendra quand la promesse aura été remplie. Lorsque le locuteur dévolle ce résultat, î est naturel de croire quill a fini de parler et que la parole est libre, linterlocuteur pouvant alors prendre la relève.

\subsubsection{LES INDICES DE JUGEMENT DE VALEUR}

Les jugements de valeur sont en quelque sorte des indices de conclusion en ce sens où ils sont généralement situés vers la fin d'un tour de parole. Ce qu'on a considéré comme étant des jugements de valeur sont des mots que le locuteur prononce pour évaluer le récit ou plutôt les faits qu'il venait d'énoncer. Par exemple, dans un tour où le locuteur parle de tirer à la carabine de calibre 22 sur des petits animaux : « J'pense y chassait l'orignal avec ça. J'tirais sur un siffleux. $Y$ serait pas resté grand-chose» (tour 6). Le locuteur évalue alors ce qui serait arrivé s'll avait atteint sa cible avec sa carabine.

\subsubsection{LES INDICES DE REDONDANCE}

La redondance ou plutôt « l'absence dinformations nouvelles » est le moment où le discours a tendance à devenir complètement paraphrastique à la fin du tour de parole long. L'absence d'informations nouvelles est caractérisée par la reprise presque intégrale d'un segment du tour de parole alors que ces reprises ne sont pas des hésitations. Par exemple: «...Pis y avait plus de place pour la mettre, l'eau... » ce segment a été repris par : «Y savait plus où la mettre » (tour 7). Plusieurs tours de parole finissent par des reformulations de ce type; ce qui peut entraîner une reprise de ce genre est que le locuteur n'a plus rien de nouveau à ajouter sur ce sujet et peut-être qu'à ce moment il laisserait sous-entendre que le tour de parole est maintenant libéré, l'interlocuteur pouvant prendre la parole. 


\subsubsection{LA PRÉSENCE DE SIGNAUX EXPLICTTES DE FINALTTÉ ET LES MOTS CÉS}

La présence dun signal explicite de finalité ou dư mot dé est probablement lindice pragmatique le plus facile à déceler. Il s'agit d'une question posée à IInterlocuteur ou bien d'un mot qui exprime directement et explicitement la finalité. Pour ce qui est de la question, des éclaircissements supplémentaires ne sont pas nécessaires. En contrepartie, il semble important d'expliquer un peu plus ce qu'est un mot clé. Ce genre dindice pourrait représenter un indice de conclusion sauf qu'il semblait bon de le distinguer des autres puisqu'll ne fonctionne pas exactement de la même façon. Les mots clés sont des mots comme finalement, ou ayant le sens de «j'ai fini de parler et tu peux prendre la parole » qui sont situés dans la dernière partie du tour de parole. Cette situation est idéale dans lidentification des fins de tour de parole, mais elle se falt plutôt rare.

Tous ces différents facteurs autant prosodiques que paradiscursifs, sémanticopragmatlques et syntaxiques ont été relevés à l'écoute afin d'étudier le plus fidèlement possible ce qui peut signaler la fin d'un tour de parole et pour en tirer les conclusions les plus justes possible. Une fois cette étape faite, nous sommes passée à l'étape suivante, qui est l'analyse instrumentale.

\subsection{L'aNALYSE INSTRUMENTALE}

\subsubsection{La numérisation}

Le corpus, après avoir subi l'analyse auditive préalable, a été numérisé à l'aide du logiciel de traitement du signal CSL (Computerized Speech Lab) version 5.03 de Kay Elemetrics, à un taux de $10000 \mathrm{~Hz}$. 


\subsubsection{La segmentation}

La segmentation du corpus a été faite de façon assez particulière, surtout en ce quil concerne le choix des voyelles segmentées. En effet, puisque nous cherchions principalement à identifier des éléments qui marquent la fin, seul un certain nombre de voyelles cibles ont été déllmitées avec précision :

- la première voyelle de chaque énoncé (donc du tour)

- l'antépénultième de chaque énoncé

- la pénultlème de chaque énoncé

- la voyelle finale de chaque énoncé (donc du tour)

Cela nous donne en général 4 voyelles cibles par énoncé. Parfois nous en obtenions moins quand l'énoncé était trop court ou que la voyelle finale était inaudible. La délimitation des voyelles a été faite à l'aide du spectrogramme et de l'oscillogramme de chacune des voyelles obtenus avec le logiciel CSL.

\subsubsection{LA DÉLIMTTATION DES PAUSES}

En même temps que la délimitation des voyelles, nous avons fait la délimitation des pauses. Contrairement aux voyelles, les pauses n'ont pas eu à être ciblées puisque toutes les pauses des locuteurs étaient relevées. C'est d'ailleurs à partir de ces dernières que les voyelles cibles ont été identifiées dans la plupart des cas. La durée des pauses qui sont présentes à l'intérieur des tours comme celle des pauses finales d'énoncé et de tour ont alors été mesurées. Cela nous permettra de vérifier s'il y a une quelconque différence entre les pauses qui sont à lintérieur des énoncés, les pauses qui sont à la fin des énoncés et les pauses qui sont à la fin des tours de parole. 


\subsubsection{LES PROBLEMES DE SEGMENTATION}

Lors de la délimitation des voyelles cibles, un problème a été rencontré à quelques reprises, soit celui des fins des énoncés qui sont inaudibles. En effet, les voyelles finales qui avalent une fo finale faible ainsi qu'une baisse d'énergie importante n'étaient pas bien relevées par le logiciel, ce qui représentait un réel obstacle. Pour contoumer ce problème, la voyelle audible qui précède la voyelle inaudible est dans ces quelques cas celle qui est considérée comme finale.

\subsubsection{Les mesures de fréquence et d'intensité}

Les mesures de fréquence et dintensité ont été prises à l'aide du logiciel CSL pour chaque voyelle aux 2/3 de la durée, ce qui correspond selon plusieurs travaux, dont celul de Di Cristo (1985), au point le plus stable et le plus représentatif de la voyelle. La hauteur de la fréquence a été notée en $\mathrm{Hz}$ et la mesure d'intensité en dB.

\subsubsection{Les mesures de la durée}

Outre les valeurs de fréquence et dintensité, la durée des voyelles a été aussi relevée. Le calcul de la durée a été également fait pour les pauses afin de pouvoir comparer les durées des différents types de pauses qui sont présentes dans un tour de parole. Les mesures de durée ont été prises à partir de la délimitation préalablement effectuée des pauses et des voyelles. Pour les voyelles, la marque de début et de fin est utilisée pour calculer la durée. Pour les pauses qui sont à l'intérieur des énoncés, elles commencent quand le locuteur arrête de parler et elles finissent quand il reprend son émission vocale. Pour les pauses finales d'énoncés ou de tour, elles commencent quand le locuteur termine son énoncé et 
elles finissent aussitôt que l'énoncé suivant débute ou que linterlocuteur émet un son, quil fournisse un signal de back-channel ou quil prenne le tour de parole.

La durée des tours de parole a été également calculée. La technique utilisée est très similaire à celle de la mesure de la durée des pauses. À l'aide du logiciel CSL, nous marquions le début du tour (le moment exact où le locuteur commençait à parler) et la fin du tour (la fin de la pause finale au moment où linterlocuteur prenait la parole). Nous pouvions avoir alors une mesure très exacte de la durée du tour.

\subsubsection{L'analyse des données}

Cette analyse a été faite à partir des données recueillies lors du relevé des valeurs de fréquence et d'intensité des voyelles cibles. Toutes les valeurs ont été classées dans un fichier Microsoft Excel pour, par la suite, en extraire les valeurs moyennes de durée des pauses et des voyelles, des énoncés et des tours, de même que la moyenne des variations de fréquence et d'intensité des voyelles cibles.

\subsubsection{LES MOYENNES}

Le calcul des moyennes a été fait pour les trois types dindices considérés, soit les valeurs de pause, de fréquence et d'intensité. Tout d'abord, la durée moyenne des trois différents types de pauses (pause interne, pause d'énoncé, pause de fin de tour) a été calculée à partir des valeurs de durée recueillies avec CSL. Par la suite, nous avons calculé la valeur moyenne de fréquence et dintensité de chaque énoncé de chaque tour. Par la suite, nous avons calculé la moyenne de fréquence des débuts d'énoncé et également la moyenne de fréquence des fins d'énoncé. 


\subsubsection{LA CHUTE DE FRÉQUENCE ET D'INTENSTIÉ}

Toujours à l'aide du logiciel Microsoft Excel, d'autres données ont été vérifiées. Étant donné limportance que peut prendre une variation de fréquence et d'intensité entre deux voyelles mesurées, le calcul de la chute de fréquence et d'intensité entre la voyelle pénultième et la voyelle finale de chaque énoncé aussi a été effectué.

\subsubsection{LES GRAPHIQUES ET LES TABLEAUX}

Toutes ces vérifications et ces calculs de moyennes sont représentés sous forme de graphiques ou de tableaux qui sont commentés dans la partie Analyse des résultats. 


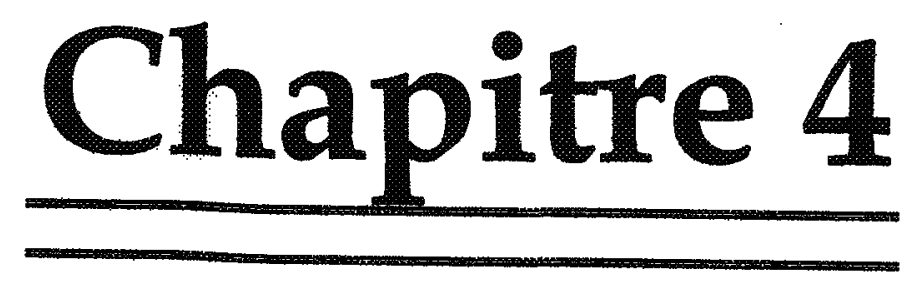

Analyse des résultats 


\subsection{INTRODUCro:}

Dans cette partie, nous analysons l'un après l'autre tous les indices que nous avons identifiés précédemment afin de bien vérifier leur utilisation dans le tour de parole en tant qu'indice de fin. Il est souvent question de comparaison entre la finale de tour et la finale d'énoncé afin de valider les résultats obtenus. Tout d'abord, nous abordons les indices prosodiques pause, intonation et intensité. Le rôle des indices non prosodiques comme la syntaxe et les indices sémantico-pragmatiques sera pris en considération dans un second temps.

\subsection{LES INDICES PROSODIQUES}

Le rôle des facteurs prosodiques dans la perception de la fin d'un tour de parole constitue le centre d'intérêt de notre recherche. Il va alors de soi qu'une importante partie de l'analyse des résultats est consacrée à ces indices. L'ordre de présentation et d'analyse de ces éléments n'est pas non plus aléatoire. Nous nous pencherons alors tout d'abord sur la présence des pauses pour, par la suite, étudier le phénomène de lintonation par les valeurs de fréquence fondamentale (FO). Dans un troisième temps, nous bonifierons cette analyse par l'examen des valeurs d'intensité associées aux voyelles de fin de tour ainsi qu'aux voyelles de fin d'énoncé.

\subsubsection{Les pauses}

La pause représente un élément important dans la production d'une élocution. Elle a plusieurs fonctions et sert, entre autres, à reprendre son souffle, à avaler sa salive et surtout à marquer l'organisation de sa pensée quand ce n'est pas à procéder à cette organisation. De ce fait, nous avons vérifié la présence ou l'absence de pauses afin de relever les éléments prosodiques et non prosodiques 
s'y rattachant. Cependant, nous ne nous sommes pas contentée de considérer la pause unlquement en tant qu'emplacement propice à l'apparition de facteurs linguistiques divers pouvant servir à notre étude. Nous avons aussi jugé utile de mesurer la durée de ces pauses et de les catégoriser selon leur emplacement dans le discours. En effet, Swerts et Geluykens (1994) ont déjà fait I'hypothèse que les sujets avaient tendance à associer une pause longue avec une frontière importante du discours. Plus spécifiquement encore, Rémi-Giraud (1987) fait le lien entre la présence d'une pause forte et les limites de l'échange. Cette tendance à associer la pause longue avec la finalité a piqué notre curiosité et nous avons cru bon nous y attarder en mesurant la durée des pauses afin de vérifier si les locuteurs de notre corpus interprètent la présence d'une pause longue comme un signal leur indiquant de prendre la parole.

\subsubsection{LES TYPES DE PAUSES}

Nous avons vu précédemment qu'il existait deux types de pauses, les unes silencieuses, les autres remplies. Seules les premières peuvent être considérées comme un indice de fin, les pauses remplies étant plutôt vues comme un moyen de garder la parole. Les pauses relevées ont été divisées en trois catégories selon leur emplacement dans le discours : pause d'intérieur, pause d'énoncé et pause de fin de tour. A priori, nous avons examiné systématiquement toutes les frontières possibles situées entre les énoncés et celles situées à la fin des tours de parole. Cette façon de faire incluait forcément les cas où il n'y avait pas de pause mesurable ( 16 cas chez le locuteur $H$ et 9 cas chez le locuteur $F$ pour les pauses d'énoncé ; 1 cas et 2 cas respectivement pour les pauses de fin de tour). Pour nos calculs, nous notions alors que la durée de la pause était de zéro seconde, ce qui peut sembler un peu particulier. Nous sommes consciente que ces pauses, que nous appelons «zéros», peuvent en quelque sorte changer ou brouiller nos résultats de comparaison puisque, dans le cas des pauses dintérieur, nous n'avons pas de valeur de «zéro », le statut de ce type de pauses étant fondamentalement 
différent de celui des deux autres types. La pause interne n'étant pas liée à des contraintes structurelles (complétude syntaxique et sémantique), discursives (changement de topique) ou conversationnelles (changement de locuteur), elle n'est pas en quelque sorte «prédéfinie » et son identification s'est faite sur des bases strictement perceptives. En l'absence d'un sience de durée perceptible, aucune pause n'est identifiée ; aucune valeur « zéro » ne peut donc être attribuée.

Tableau 1 : Durées moyennes des pauses (en cs) pour chaque locuteur selon le type de pauses incluant ou non les « zéros $»$

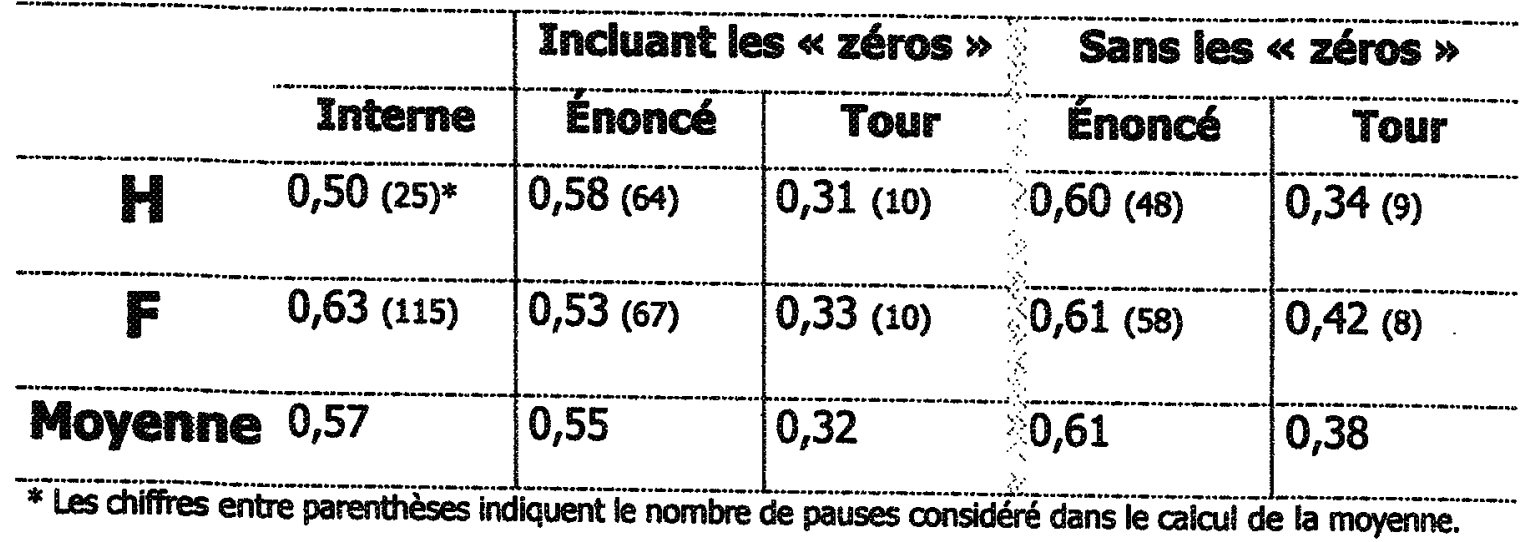

Le Tableau 1 nous montre les durées moyennes obtenues pour chaque locuteur selon le type de pauses. Dans la partie de gauche, on voit les moyennes qui incluent les «zéros». On peut y remarquer que les pauses de fin de tour sont beaucoup plus courtes que les autres types de pauses. Pour le locuteur $\mathrm{H}$, les pauses de fin de tour sont en moyenne presque deux fois plus courtes que les pauses d'énoncé et pratiquement une fois et demie plus courtes que les pauses dintérieur. Pour le locuteur $F$, on y retrouve un phénomène similaire sauf que cette fois-ci, les pauses les plus longues sont les pauses dintérieur. Tout cela semble aller à l'encontre de l'hypothèse de départ que nous avons vue précédemment quant à la relation entre la durée des pauses et l'importance des frontières de Swerts et Geluykens (1994) et des observations de Rémi-Giraud (1987) qui semblent abonder dans le même sens. 
Pour être conformes à cette hypothèse, les pauses de fin de tour auraient dû être plus longues que les pauses de fin doénoncé, elles-mêmes pius longues que les pauses dintérieur. Or, non seulement les pauses de fin de tour sont-elles les plus courtes, mais ce sont (dans le cas de F) les pauses dintérieur quil sont les plus longues. Il n'est cependant pas impensable que ce soit dans ce dernier cas l'effet d'un artifice de calcul, la présence de valeurs zéro - non existantes, rappelons-le pour les pauses d'intérieur - contribuant à diminuer la durée moyenne des pauses de fin d'énoncé et de fin de tour.

Dans la partie droite du Tableau 1, nous retrouvons les durées moyennes des pauses pour chaque locuteur, mais cette fois-ci les valeurs « zéro » ont été retirées du calcul. On peut alors remarquer que les résultats ne diffèrent pas beaucoup de ceux obtenus précédemment, car les proportions demeurent somme toute les mêmes. Les pauses d'énoncé sont encore en moyenne globalement les plus longues et les pauses de fin de tour sont encore les plus courtes. Il faut aussi noter que dans le cas de $F$, le type de pauses le plus long est encore une fols celui des pauses d'intérieur, mais la différence avec les pauses de fin d'énoncé devient relativement marginale.

Des résultats similaires ont été obtenus dans Swerts (1997) et Swerts et Geluykens (1994), résultats qui, rappelons-le, allaient à l'encontre de leur hypothèse initiale. Les chercheurs nous expliquent alors que la durée des pauses serait en quelque sorte dépendante de la structure en topique du discours et non de la structure syntaxique, donc que les pauses les plus longues étaient celles situées tout de suite après lintroduction d'un nouveau topique et entre les instructions ${ }^{4}$. Les pauses permettraient ainsi au locuteur d'organiser et de structurer son tour de parole. Ils avancent alors lidée que la durée des pauses diminue en fonction de la

\footnotetext{
4 Dans une tâche de description d'œurres d'art ou une tâche d'assemblage élaborée par Swerts (1997) et Swerts et Geluykens (1994), les instructions représentent un degré de structure qui peut être l'équivalent des énoncés de notre corpus.
} 
baisse du degré dincertitude du discours. Ten Bosch, Oostdijk et Boves (2005), dans une étude récente qui porte entre autres sur la durée des pauses entre les tours de parole, en arrivent à des résultats simllaires.

L'explication que nous proposons n'est pas sans relation avec celle de Swerts et Geluykens (1994), mais nous devons en plus y ajouter l'aspect interactionnel qui est omniprésent dans la conversation. Les tours de parole longs de notre corpus sont produits dans une situation de conversation amicale, linterlocuteur est donc enclin à laisser parler le locuteur. Dans ces conditions, les locuteurs ne sont pas soumis à beaucoup de pressions pour conserver leur tour, contrairement à ce quil se produit pour une conversation de type argumentatif ou un débat. Les pauses longues, généralement produites selon nos résultats en interne et en fin d'énoncé, ne posent pas de réels dangers à la conservation du tour de parole. Linterlocuteur sent, selon le déroulement de la conversation, que le locuteur n'a pas fini de parler et, puisqu'il n'est pas en situation d'argumentation, semble accorder une plus grande attention aux indices de non-conclusion qui sont produits par le locuteur, lorsqu'une pause est précédée d'un de ces indices, l'interlocuteur s'attend donc à ce que le locuteur poursuive sur son sujet. II ne faut pas oublier non plus limportance que peut avoir le rôle des participants dans la situation expérimentale, c'est-à-dire que, dans les conversations étudiées, les deux personnes sont conscientes qu'elles ont un rôle à jouer, celui de lintervieweur et celui de linterviewé.

Par ailleurs, puisque nous avons affaire à des tours de parole longs, le facteur temps, jumelé au déroulement du topique, pourrait aussi expliquer la plus faible durée des pauses entre les changements de tour de parole. En effet, il est vraisemblable de croire qu'après plusieurs secondes au rôle d'interiocuteur, le sujet n'attendra pas très longtemps après l'ouverture d'une fenêtre d'opportunité que constitue la pause avant de prendre la parole, cela pour éviter que le locuteur ne reprenne le « plancher » en enchaînant sur un autre sujet, surtout si cette fenêtre 
s'accompagne d'indices de conclusion. De pus, la conclusion du topique actuel est généralement un bon indice que le locuteur est sur le point de céder la parole.

En fali, on sent très bien ici que plusieurs facteurs peuvent s'additionner et intervenir conjointement dans la perception de la finalité. Cela viendrait alors peutêtre expliquer la raison pour laquelle les pauses finales sont en moyenne plus courtes que les autres types de pauses; tous les indices qui peuvent être émis permettent ainsi à linterlocuteur de comprendre que la fin du tour actuel approche et de se préparer à prendre la parole dès qu'il sent une opportunité, ce que Sacks, Schegloff et Jefferson (1974) et ten Bosch, Oostdijk et Boves (2005) appellent une «transition-relevance place».

\subsubsection{Lintonation}

\subsubsection{LES INDICES GLOBAUX}

L'étude de lintonation dans ce travall s'est faite sur trois plans. Tout d'abord, nous nous sommes intéressée aux variations intonatives dans une mesure plus générale, c'est-à-dire aux indices de fréquence globaux, puis à ceux qu'on pourrait qualifier dintermédiaires ou de sectoriels. Finalement, ce fut au tour des variations intonatives localisées à la fin des énoncés et des tours de parole. Du point de vue plus général, nous avons examiné les indices intonatifs globaux des tours de parole. Nos observations ont alors porté sur la comparaison des valeurs moyennes de F0 des voyelles cibles des énoncés et des tours de parole. Par la suite, dans une perspective intermédiaire ou sectorielle, nous avons comparé, pour les énoncés comme pour les tours, les valeurs de FO des voyelles finales avec les moyennes des voyelles cibles des énoncés du tour. Enfin, du point de vue des indices intonatifs locaux, les schémas intonatifs des fins d'énoncé et de tour ont été plus particulièrement examinés. 
Bien que l'alternance entre les locuteurs soit dominante dans le type de discours que nous étudions, il n'en demeure pas" moins que le but ultime est de communiquer un message. Ce message, quel qu'en soit le sujet, est fortement soutenu par la courbe intonative que le locuteur produit simultanément. Plusieurs chercheurs affirment cependant que peu importe les mouvements locaux dintonation, la FO aurait une forte tendance à descendre au fur et à mesure que le discours avance (Swerts, Collier et Terken, 1994; Lieberman, Katz, Jongman, Zimmerman, Miller, 1985 et Lehiste, 1975). Même si les données que nous avons recueillies ne nous permettent pas d'étudier directement la llgne de déclinaison de la Fo, telle que décrite dans Collier et $t$ Hart (1975) ainsi que dans Pierrehumbert (1979), certaines observations sur l'évolution générale de la fréquence peuvent cependant être faites.

Le Tableau 2 présente les valeurs de fréquence des voyelles finales de tour en comparaison avec les valeurs moyennes de Fo des énoncés de chaque tour correspondant. Il est très intéressant de constater dans le sommaire du Tableau 2 que la majorité des valeurs des voyelles finales de tour se retrouve en bas de la valeur moyenne de FO du tour, c'est-à-dire 13 voyelles sur 20 (65\% des cas). Sans confirmer l'existence d'une descente générale de FO dans les tours de parole de notre corpus, ce résultat refiète tout de même une tendance à produire une voyelle plus basse en fin de tour. 
Tableau 2 : Comparaison de la valear de Fo de la voyelle finale de rour par raport movene de Fo des voyelles cibles des éncncts du cour de parole

\begin{tabular}{|c|c|c|c|c|c|c|c|}
\hline $\begin{array}{l}\text { Tour de } \\
\text { parole }\end{array}$ & $\begin{array}{c}\text { Fo } \\
\text { voy. } \\
\text { finale }\end{array}$ & $\begin{array}{l}\text { moy. Fo } \\
\text { énoncés } \\
\text { du cour }\end{array}$ & $t=5$ & $\begin{array}{l}\text { Jour de } \\
\text { parole }\end{array}$ & $\begin{array}{c}\text { ro } \\
\text { voy. } \\
\text { finale }\end{array}$ & $\begin{array}{l}\text { moy. Fo } \\
\text { énoncés } \\
\text { du tour }\end{array}$ & $\begin{array}{l}+1 \\
1=\end{array}$ \\
\hline$H-01$ & 82 & 114,08 & - & $8-01$ & 76 & 188,04 & - \\
\hline H.02 & 121 & 112,13 & + & $F-02$ & 179 & 172,38 & $=$ \\
\hline$H=03$ & 125 & 137,35 & $=$ & $F=03$ & 196 & 169,1 & + \\
\hline$H=04$ & 105 & 111,77 & $=$ & $F-04$ & $20 \overline{8}$ & 156,9 & + \\
\hline$M=05$ & 115 & 106,2 & + & $F-05$ & 156 & 164,56 & $=$ \\
\hline H-06 & 97 & 115,14 & $=$ & F-06 & 145 & 172,06 & - \\
\hline $\mathrm{H}=07$ & 96 & 110,54 & - & $F-07$ & 97 & 157,32 & $=$ \\
\hline H-08 & 133 & 108,08 & + & $F-08$ & 172 & 207,36 & $\because$ \\
\hline $\mathrm{H}-09$ & 91 & 117 &. & $F-09$ & 182 & $222,23^{\circ}$ & 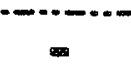 \\
\hline $\mathrm{H}-10$ & 104 & 118,33 & & $F-10$ & 217 & 194,24 & $T$ \\
\hline
\end{tabular}

\begin{tabular}{|c|c|c|c|c|}
\hline & $\begin{array}{l}\text { Voyelle inale } \\
\text { inférieure }(-) \text { à la } \\
\text { moy. Fo des énoncés } \\
\text { du tour }\end{array}$ & $\begin{array}{l}\text { Voyelle finale } \\
\text { moýrieure ( }+ \text { ) à la } \\
\text { du tour ences }\end{array}$ & $\begin{array}{l}\text { Voyelle finale égale } \\
\text { (=) à la moy. Fo des } \\
\text { énoncés du tour }\end{array}$ & $\begin{array}{l}\text { Total des } \\
\text { énonces }\end{array}$ \\
\hline nombre & 13 & 6 & 1 & 20 \\
\hline$\%$ & $65 \%$ & $30 \%$ & $5 \%$ & $100 \%$ \\
\hline
\end{tabular}

Le Tableau 3 qui suit présente les résultats de la comparaison non pius pour la seule voyelle finale, mais pour la moyenne des voyelles cibles de l'énoncé final de tour, ce qui peut donner une image plus révélatrice du mouvement de déclinaison sur l'ensemble du tour. Le sommaire montre que si les cas où il $n^{\prime} y$ a pas de distinction entre les fins de tour et les énoncés intratour totalisent $50 \%$, les voyelles cibles de l'énoncé final de tour présentent néanmoins une fréquence plus

\footnotetext{
${ }^{5}$ Nous considérons, ici, qu'un écart d'au moins $5 \%$ entre les deux valeurs est nécessaire pour que la différence solt perceptible (Rossi, 1999). Si ce seull n'est pas atteint, les deux valeurs sont traitées comme égales.
} 
basse que les autres voyelles cibles du tour dans $45 \%$ des cas, les écarts allant de $7 \%$ jusquà $27 \%$.

Tableau 3 : Comparalson de la moyenne de fo des voyelles dbles de l'énoncé final par rapport a celle des autres énoncés du tour

\begin{tabular}{|c|c|c|c|c|c|c|c|}
\hline $\begin{array}{l}\text { Tour de } \\
\text { parole }\end{array}$ & $\begin{array}{l}\text { moy. Fo } \\
\text { énoncé } \\
\text { final }\end{array}$ & $\begin{array}{l}\text { moy. Fó } \\
\text { énoncés } \\
\text { du tour }\end{array}$ & $1=$ & $\begin{array}{l}\text { Tour de } \\
\text { parole }\end{array}$ & $\begin{array}{l}\text { moy. Fó } \\
\text { énoncé } \\
\text { final }\end{array}$ & $\begin{array}{l}\text { moy. Fo } \\
\text { énoncés } \\
\text { du tour }\end{array}$ & $1=$ \\
\hline H-01 & 85,5 & 114,08 & $=$ & $F=01$ & 142,5 & 188,04 & 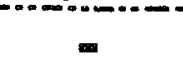 \\
\hline $\mathrm{H}=02$ & 104 & 112,13 & - & F-02 & 178,5 & 172,38 & \\
\hline $\mathrm{H}=03$ & 125 & 137,35 & - & $F=03$ & 175 & 169,1 & \\
\hline H-04 & 114,25 & 111,77 & $=$ & $\bar{F}=04$ & 159,25 & 156,9 & \\
\hline H-05 & 108 & 106,2 & $=$ & $\bar{F}-05$ & 158 & 164,56 & $=$ \\
\hline H-06 & 102 & 115,14 & 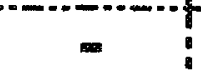 & F.06 & 135,5 & 172,06 & \\
\hline $\mathrm{H}-07$ & 97,25 & 110,54 & - & $F-07$ & 115,3 & 157,32 & $=$ \\
\hline $4-08$ & 106,75 & 108,08 & $=$ & $F=08$ & 177,5 & 207,36 & 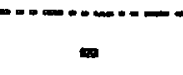 \\
\hline$H-09$ & 125,25 & 117 & + & $F=09$ & 212,75 & 222,23 & $=$ \\
\hline$H-10$ & 114,5 & 118,33 & $=$ & $F-10$ & 199,75 & 194,24 & $=$ \\
\hline \multicolumn{8}{|c|}{ sommaire } \\
\hline & \multicolumn{2}{|c|}{$\begin{array}{l}\text { Moy. Fo énoncé final } \\
\text { inférieure (-) à la } \\
\text { moy. fo des énoncés } \\
\text { du tour }\end{array}$} & \multicolumn{2}{|c|}{$\begin{array}{l}\text { Moy. Fo énoncé finail } \\
\text { supérieure }(+) \text { à la } \\
\text { moy. Fo des énoncés } \\
\text { du tour }\end{array}$} & \multicolumn{2}{|c|}{$\begin{array}{l}\text { Moy. Fo énoncé rinai } \\
\text { égale ( }=\text { a la moy. } \\
\text { Fo des énoncés du } \\
\text { tour }\end{array}$} & $\begin{array}{l}\text { Total des } \\
\text { énoncés }\end{array}$ \\
\hline ombre & & & & \multirow{2}{*}{\multicolumn{2}{|c|}{10}} & 20. \\
\hline$\ldots$ & & & & & & & $100 \%$ \\
\hline
\end{tabular}

Tout aussi intéressant est le fait qu'on ne trouve qu'un cas sur vingt pour lequel les valeurs de l'énoncé final sont supérieures à celles de la moyenne des autres énoncés du tour. Sans confirmer I'mypothèse d'une descente de Fo tout au long du tour de parole, les cas d'égalité étant les plus nombreux (50\%), ces résultats ne la contredisent pas non plus puisque les cas de fréquence inférieure représentent tout de même $45 \%$ contre un seul cas de fréquence supérieure en finale de tour. 
Le Tableau 4 qui rassemble les résultats de la comparaison de la fréquence des voyelles finales de tour avec la moyeme des valeurs pour les voyelles finales des énoncés qui composent le tour donne des résultats encore plus explicites. Il s'agit là de résultats d'autant plus révélateurs que la voyelle finale de tour est ici comparée à ce qui lui ressemble le plus en même temps que ce à quoi elle s'oppose directement et dont elle cherche à se distinguer, c'est-à-dire les voyelles finales qui ne sont pas des finales de tour.

Tableau 4: comparaison de la valeur de Fo (en Hz) de la voyelle male de tour par rapport à la moyenne de fo des voyelles de fin d'énoncé d'un même tour de parole

\begin{tabular}{|c|c|c|c|c|c|c|c|}
\hline $\begin{array}{l}\text { Tour de } \\
\text { parole }\end{array}$ & $\begin{array}{l}\text { Moy. Fo } \\
\text { des voy. } \\
\text { de in } \\
\text { d'énonoes }\end{array}$ & $\begin{array}{l}\text { Val. en } \\
\text { Hz de la } \\
\text { voy. } \\
\text { finale }\end{array}$ & $\begin{array}{c}+1= \\
1=\end{array}$ & $\begin{array}{l}\text { Tour de } \\
\text { parole }\end{array}$ & $\begin{array}{l}\text { Moye fo } \\
\text { des voy. } \\
\text { deñn } \\
\text { d'énonce }\end{array}$ & $\begin{array}{l}\text { Val. en } \\
\text { Hz de la } \\
\text { voy. } \\
\text { fnale }\end{array}$ & $\begin{array}{l}+1= \\
1=\end{array}$ \\
\hline $\mathrm{H}-01$ & 111 & 82 & $\ldots$ & $F=01$ & 193 & 76 & $=$ \\
\hline H-02 & 120 & 121 & $=$ & $F-02$ & 166 & 179 & + \\
\hline $\mathrm{H}-03$ & 131 & 125 & - & $F-03$ & 196 & 196 & $=$ \\
\hline$H=04$ & 113 & 105 & - & $F-04$ & 141 & 208 & + \\
\hline H-05 & 106 & $11 \overline{5}$ & + & $F-05$ & 178 & 156 & - \\
\hline H-06 & 120 & 97 & $=$ & $F=06$ & 186 & 145 & - \\
\hline $\mathrm{H}-07$ & 111 & 96 & $=$ & $F=07$ & 138 & 97 & - \\
\hline $\mathrm{H}=08$ & 108 & 133 & + & $F-08$ & 212 & 172 & . \\
\hline H-09 & 126 & 91 & - & F-09 & 222 & 182 & . \\
\hline$H-10$ & 112 & 104 & - & $F-10$ & 209 & 217 & + \\
\hline \multicolumn{8}{|c|}{ sommaire } \\
\hline & \multicolumn{2}{|c|}{$\begin{array}{l}\text { Val. en Hz de la voy. } \\
\text { finale inférieure (-) } \\
\text { à la moy. Fo voy. } \\
\text { finale d'énoncés. }\end{array}$} & \multicolumn{2}{|c|}{$\begin{array}{l}\text { val. en Hz de la voy. } \\
\text { finale supérieure (t) } \\
\text { a la moy. Fo voy. } \\
\text { finale d'énoncés }\end{array}$} & \multicolumn{2}{|c|}{$\begin{array}{l}\text { Val. en Hz de la voy. } \\
\text { finale égale ( } \text { ) a la } \\
\text { moy. Fo voy. finale } \\
\text { d'énoncés }\end{array}$} & $\begin{array}{l}\text { Total des } \\
\text { énoncés }\end{array}$ \\
\hline nombre & \multicolumn{2}{|c|}{13} & \multirow{2}{*}{\multicolumn{2}{|c|}{$25 \%$}} & \multirow{2}{*}{\multicolumn{2}{|c|}{2}} & 20. \\
\hline$\%$ & \multicolumn{2}{|c|}{$65 \%$} & & & & & $100 \%$ \\
\hline
\end{tabular}


Le sommaire montre quill y a 13 tours sur 20 , soit $65 \%$ des cas, pour lesquels la voyelle inale du tour affiche une valeur de fréquence sensiblement inférieure à la moyenne de finales d'énoncé, contre $25 \%$ pour les cas de fréquence supérleure. L'ampleur de cette différence varie de $-5 \%$ à $-61 \%$. Il faut cependant faire attention quill s'agit là d'une tendance générale basée sur la comparaison avec la moyenne; dans le détall, il n'y a en fait que trois tours (H-09, F-08 et F-09) pour lesquels la voyelle finale de tour présente, de façon absolue, la valeur de fréquence la plus basse de toutes les voyelles finales.

II faut noter également que ces résultats globaux sont indépendants de l'orientation individuelle de la courbe intonative présente dans l'énoncé final ou plus précisément sur les voyelles finales. Il peut donc arriver qu'un énoncé de fin de tour se termine sur une intonation montante à la fin, mais que la valeur de la Fo finale demeure tout de même inférieure à la valeur moyenne de FO du tour. À première vue, cette situation peut sembler paradoxale, mais dans une perspective où II y a réellement une descente globale de la FO tout au long du tour, il est fort possible qu'une légère montée finale nous situe à un niveau plus bas que la valeur moyenne de FO du tour. La montée joue alors au plan strictement local. C'est le cas de trois tours sur vingt tours chez le même locuteur, plus précisément : F-05, $\mathrm{F}-07$ et $\mathrm{F}-09$.

\subsubsection{LES INDICES INTERMÉDIAIRES OU SECTORIELS}

Dans une perspective un peu moins globale, il semble intéressant de reprendre, dans le cas de l'énoncé, les mêmes comparaisons que celles faites pour le tour de parole afin de voir s'il existe des différences de comportement. Le Tableau 4 nous montre la proportion de voyelles finales de l'énoncé qui ont une fréquence inférjeure, supérieure ou égale à la moyenne des autres voyelles cibles de l'énoncé. Ce tableau est le résultat de la comparaison des voyelles finales de tous 
les énoncés présents dans le corpus avec les voyelles cibles de l'énoncé dont elles font partie.

Tableau 5: La proportion de voyelles finales d'énoncé dont la fréquence est inférieure, supérieure ou égale la la moyenne de tréquence des voyelles cibles de I'énonó correspondant (incluant g'énoncé final de tour) ${ }^{6}$

\begin{tabular}{|c|c|c|c|c|}
\hline & $\begin{array}{l}\text { voyelle } \\
\text { finale } \\
\text { d'énoncé } \\
\text { Inférieure }\end{array}$ & $\begin{array}{l}\text { voyelle } \\
\text { finale } \\
\text { d'énoncé } \\
\text { supérieure }\end{array}$ & $\begin{array}{l}\text { voyelle } \\
\text { finale } \\
\text { d'énonce } \\
\text { égale }\end{array}$ & $\begin{array}{l}\text { nombre } \\
\text { total } \\
\text { d'énoncés }\end{array}$ \\
\hline H & 24 & 24 & 26 & 74 \\
\hline$F$ & 29 & 34 & 14 & 77 \\
\hline ToraL & 53 & 58 & 40 & 151 \\
\hline$\%$ & $351 \%$ & $38,4 \%$ & $26,5 \%$ & $100 \%$ \\
\hline
\end{tabular}

On peut remarquer, sur l'ensemble des énoncés du corpus, quill n'y a pas de situation nettement dominante, même si on observe une très légère préférence à produire des voyelles finales d'énoncé avec une FO supérieure à la FO moyenne de l'énoncé dont elles font partie $(38,4 \%)$. Il ne faut cependant pas perdre de vue que le cas inverse, soit celui où la FO de la voyelle finale est inférieure à la moyenne de l'énoncé est presque aussi fréquent avec $35,1 \%$ des cas. Enfin, la FO de la voyelle finale est sensiblement égale à la valeur moyenne de l'énoncé compte tenu du seuil différentiel minimal de $5 \%$, dans $26,5 \%$ des cas. On ne peut donc considérer quiil se dégage une tendance réelle. Il faut cependant éviter de conclure trop rapidement des données du Tableau 5 puisque tous les énoncés du corpus ont été comptabllisés sans faire de distinction quant à leur emplacement, final ou intérieur, dans le tour. Nous avons cru bon de présenter les deux types d'énoncé dans des tableaux distincts. Le Tableau 5.1 montre les résultats pour tous les énoncés intratour (énoncés qui sont présents à l'intérieur du tour incluant l'énoncé initial) et le Tableau 5.2 et son sommaire ne retiennent que les résultats des énoncés finals de tour.

\footnotetext{
${ }^{6}$ Les valeurs et données qui ont servi pour ce tableau sont présentées à l'ANNEXE $B$.
} 


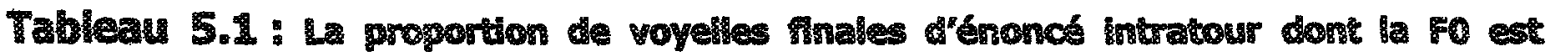
Inferteure, superieure ou egale d la moyenne des voyelles cibles des énoncés intratour correspondants

\begin{tabular}{|c|c|c|c|c|}
\hline & $\begin{array}{l}\text { voyelle } \\
\text { Inale } \\
\text { d'énonce } \\
\text { inférieure }\end{array}$ & $\begin{array}{l}\text { vovelle } \\
\text { Tinale } \\
\text { d'énoncé } \\
\text { supérieure }\end{array}$ & $\begin{array}{l}\text { voyelle } \\
\text { finale } \\
\text { d'énoncé } \\
\text { égale }\end{array}$ & $\begin{array}{l}\text { nombre } \\
\text { sotal } \\
\text { d'énoncés }\end{array}$ \\
\hline$H$ & 20 & 21 & 23 & 64 \\
\hline$F$ & 26 & 30 & 11 & 67 \\
\hline TOTAL & 46 & 51 & 34 & 131 \\
\hline$\%$ & $35,1 \%$ & $38,9 \%$ & $26 \%$ & $100 \%$ \\
\hline
\end{tabular}

Les résultats du Tableau 5.1 concernant tous les énoncés non finals sont pratiquement identiques à ceux du Tableau 5 , ce qui n'a rien de surprenant compte tenu de la faible proportion d'énoncés finals de tour par rapport à l'ensemble. Les résultats pour ces énoncés finals de tour sont présentés dans le Tableau 5.2. La comparaison du sommaire du Tableau 5.2 avec le Tableau 5.1 nous amène à conclure qu'il n'y a pas de différence notable entre le comportement de la voyelle de fin de tour et les voyelles de fin d'énoncé, du point de vue qui nous intéresse ici, c'est-à-dire le rapport entre la fréquence de la voyelle finale et la moyenne de fréquence de l'énoncé dont elle fait partie. 
Tablean 5.2: Comparison des fo des voyelles firales de tour par rapgor a ia valeur moyenne des voyelles clbles de l'énoned dont the fait partie

\begin{tabular}{|c|c|c|c|c|c|c|c|}
\hline $\begin{array}{l}\text { Tour de } \\
\text { parose }\end{array}$ & $\begin{array}{l}\text { Fo } \\
\text { voyelle } \\
\text { finale }\end{array}$ & $\begin{array}{c}\text { moy. Fo } \\
\text { énonce } \\
\text { final }\end{array}$ & $+1=$ & $\begin{array}{c}\text { Tour de } \\
\text { parole }\end{array}$ & $\begin{array}{c}\text { po } \\
\text { voyelle } \\
\text { finale }\end{array}$ & $\begin{array}{l}\text { moy. } \\
\text { énoncé } \\
\text { final }\end{array}$ & $+1=$ \\
\hline$H=01$ & 82 & 85,5 & $\equiv$ & $F-01$ & 76 & 142,5 & $\ldots$ \\
\hline $\mathrm{H}-02$ & 121 & 104 & + & $F-02$ & 179 & 178,5 & $=$ \\
\hline H-03 & 125 & 125 & $=$ & $F-03$ & 196 & 175 & + \\
\hline H-04 & 105 & 114,25 & 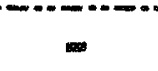 & $F-04$ & $20 \overline{8}$ & 159,25 & + \\
\hline H-05 & 115 & $108^{\circ}$ & + & $F=05$ & 156 & 158 & $=$ \\
\hline 17-06 & 97 & 102 & 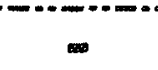 & $\bar{F}=06$ & 145 & 135,5 & + \\
\hline $11-07$ & 96 & 97,25 & $=$ & $8-07$ & 97 & 115,3 & $=$ \\
\hline $\mathrm{H}-08$ & 133 & 106,75 & + & $F-08$ & 172 & 177,5 & $=$ \\
\hline H-O & 91 & 125,25 & - & $\mathrm{F}-09$ & 182 & 212,75 & 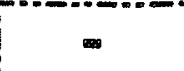 \\
\hline$H-10$ & 104 & 114,5 & $=$ & $F-10$ & 217 & 199,75 & + \\
\hline $\begin{array}{l}\text { Moy. } \\
\text { Gén. }\end{array}$ & 106,9 & 108,25 & & & 162,8 & 165,41 & \\
\hline & \multicolumn{7}{|c|}{ sommaire } \\
\hline & \multicolumn{2}{|c|}{$\begin{array}{l}\text { Fo voyelle finale de } \\
\text { tour inferieure }(-) \text { a } \\
\text { la moy. Fo de } \\
\text { l'énoncé fnnal du }\end{array}$} & \multicolumn{2}{|c|}{$\begin{array}{l}\text { Fo voyelle finale de } \\
\text { tour supérieure (+) } \\
\text { a la moy. Fo de } \\
\text { g'énoncé final du } \\
\text { tour }\end{array}$} & \multicolumn{2}{|c|}{$\begin{array}{l}\text { Fo voyelie finale de } \\
\text { tour égale }(=) \text { a la } \\
\text { moy. Fo de I'énoncé } \\
\text { final du tour }\end{array}$} & $\begin{array}{l}\text { Total des } \\
\text { énoncés }\end{array}$ \\
\hline nombre & \multicolumn{2}{|c|}{7} & \multicolumn{2}{|c|}{$35 \%$} & & 20 \\
\hline$\%$ & \multicolumn{2}{|c|}{$35 \%$} & \multicolumn{2}{|c|}{$35 \%$} & & & $100 \%$ \\
\hline
\end{tabular}

Ces résultats permettent de raffiner l'interprétation des données du Tableau 2 qui avaient fait voir une nette tendance des voyelles finales de tour à présenter une fréquence plus basse que la moyenne de fréquence des énoncés de tour (dans $65 \%$ des cas, contre $30 \%$ où la fréquence était plus haute). Il ressort sous cet éclairage que cette prédominance d'une fréquence basse en fin de tour doit être mise au compte d'un mouvement général de déclinaison tout au long du tour plutôt que du fait que les énoncés finals de tour présentent, plus fréquemment, au 
plan local qu'est celui de l'énoncé, une finale basse. Le sommaire du Tableau 5.2 fait au contraire voir une répartition à peu près égale des trois possibilités (montant, descendant, stable), répartition qui ne se différencie guère de celle que I'on observe pour les finales d'énoncé intratour (Tableau 5.1). II ne semble donc pas que l'on puisse trouver, à ce niveau intermédiaire qu'est celui du rapport de la fréquence de la voyelle finale par rapport à la fréquence moyenne de voyelles cibles de l'énoncé dont elle fait partie, un indice qui nous permettrait de reconnaître les finales de tour et de les distinguer des finales d'énoncé.

\subsubsection{LES INDICES LOCAUX}

Les indices intonatifs globaux nous ont permis d'examiner si certains phénomènes pouvaient se produire au plan général du tour de parole. Ils nous ont servi à vérifier si nous pouvions percevoir une sorte de courbe de fréquence schématisée tout au long de la production du tour de parole. Les indices sectoriels, quant à eux, concernaient surtout les moyennes de fo des énoncés. Nous avons alors fait des comparaisons de valeurs entre les énoncés finals et les énoncés intratour et leurs voyelles finales respectives. Les indices intonatifs locaux, de leur côté, visent autant l'énoncé que le tour, comme les indices sectoriels, mais d'une manière plus ciblée. Ces indices sont constitués des schémas intonatifs produits à la fin des énoncés ainsi qu'à la fin des tours. L'attention est donc principalement portée sur les valeurs de fréquence des voyelles finales d'énoncé et de tour.

\subsection{La méthode de classement des schémas intonatifs}

Pour identifier les schémas intonatifs des fins d'énoncé, nous avons utilisé les valeurs de fréquence prélevées à l'aide de CSL aux $2 / 3$ de chacune des 3 voyelles finales de chaque énoncé, c'est-à-dire l'antépénultième, la pénultième et la finale. À l'aide des valeurs numériques obtenues, nous avons pu tracer une courbe de 
fréquence pour la finale des énoncés. Nous avons identifié neuf schémas répartis dans trois catégories : les montées, les descentes et les neutres.

Pour cette partie de l'analyse, 132 énoncés du corpus, sur une possibilité de 151, ont été retenus pour le classement dans les 9 types de schémas. Lors de la prise de données sur CSL, II était parfois impossible de lire la valeur de fréquence de certaines voyelles finales pour des raisons techniques comme : la voyelle finale qui était trop courte, le locuteur qui parłait trop faiblement à la fin des énoncés ou des tours, ou bien linterlocuteur qui parlait en même temps que le locuteur. Nous n'avions d'autre choix que d'écarter ces énoncés n'ayant pas à notre disposition les valeurs des voyelles finales.

Parmi les 19 énoncés rejetés pour cette partie de l'analyse, il y avait aussi des énoncés très courts où il nous était impossible d'obtenir trois voyelles différentes. Nous avons rejeté les cas où une seule voyelle était disponible, ce qui nous aurait forcée à prendre trois fois la même voyelle. Pour les cas où, pour lidentification de la courbe du schéma intonatif, nous avons eu à prendre deux fois la valeur de la même voyelle plus une autre voyelle, incluant la voyelle finale, ils ont été traités comme les autres énoncés. Nous avons décidé de les conserver étant donné que nous avons la valeur de la voyelle finale. De plus, ces situations sont regroupées dans les schémas intonatifs qui présentent un plateau partiel et ils ne viennent pas interférer avec les autres types de schémas intonatifs.

\subsection{Les neuf schémas intonatifs}

Le Tableau 5.1 présente la répartition des énoncés finals de tour selon les 9 types de schémas intonatifs dans les 3 catégories mentionnées. Les résultats obtenus sont quelque peu différents des attentes que nous avions. Tout d'abord, on peut remarquer que deux schémas ne se retrouvent dans aucun énoncé final de notre 
corpus; ce sont les schémas montant ( ) et montant avec un plateau à la fin $(\rightarrow)$. Pour quatre de ces schémas, la répartition est assez uniforme avec un énoncé chacun. Pour deux autres schémas, on retrouve deux et trois énoncés respectivement. Ce qui nous a étonnée, c'est la grande quantité d’énoncés pour le schéma descendant-montant ( $)$. En effet, $40 \%$ des énoncés finals correspondent à ce schéma. Le bilan final pour les schémas intonatifs nous indique que plus de $53 \%$ des énoncés ont une orientation montante d'une façon ou d'une autre alors que seulement $20 \%$ des énoncés ont une orientation descendante. Les schémas intonatifs descendants arrivent même au troisième rang du classement, derrière les orientations neutres. Ces résultats sont en contradiction avec la position de Duncan (1972: 286) lorsquill avance que la fin de tour peut être tout ce qui diffère du ton neutre.

Tableau 6.1 Répartition des schémas intonatifs des voyelles antépénultièmes, pénultièmes et finales des énoncés finals de tour de parole dans les trois catégories

\begin{tabular}{|c|c|c|c|c|c|c|c|c|c|c|}
\hline & \multicolumn{3}{|c|}{ montées } & \multicolumn{3}{|c|}{ descentes } & \multicolumn{3}{|c|}{ neutres } & \multirow[t]{2}{*}{ Total } \\
\hline & 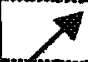 & $V$ & -7 & & $A$ & 7 & $\rightarrow$ & $M$ & $\rightarrow$ & \\
\hline H & 0 & 2 & 1 & 0 & 1 & 1 & 1 & 1 & 0 & 7 \\
\hline$\%$ & 0 & 29 & 14,2 & 0 & 14,2 & 14,2 & 14,2 & 14,2 & 0 & \\
\hline$F$ & 0 & 4 & 1 & 1 & 0 & 0 & 0 & 2 & 0 & 8 \\
\hline$\%$ & 0 & 50 & 12,5 & 12,5 & 0 & 0 & 10 & 25 & 0 & \\
\hline Globa & 0 & 6 & 2 & 1 & 1 & 1 & 1 & 3 & 0 & 15 \\
\hline \multirow[t]{2}{*}{$\%$} & 0 & 40 & 13,3 & 6,7 & 6,7 & 6,7 & 6,7 & 20 & 0 & \\
\hline & \multicolumn{3}{|c|}{$53,3 \%$} & \multicolumn{3}{|c|}{$20 \%$} & \multicolumn{3}{|c|}{$26,7 \%$} & \\
\hline
\end{tabular}

Du côté des énoncés intratour au Tableau 6.2, un scénario très semblable à celui du Tableau 6.1 se dessine. On peut voir que les montées sont encore en nette prédominance (59\%). Pour ce qui est des deux autres types de schémas, la différence n'est encore pas bien grande (environ 6\%), mais ce sont cette fois les orientations neutres qui sont les moins fréquentes avec $18,8 \%$. 
Nos résultats vont donc à l'encontre de ceux de Swerts et Collier (1992) et de Swerts, Collier et Terken (1994). En effet, lors de ces deux recherches, le schéma intonatif descendant-montant n'a pas été perçu comme final, mais a été plutôt associé à des énoncés intratour dans des situations de non-finalité ou de préfinalité. Cette différence pourrait s'expliquer par une différence quant à la sélection du type de discours (monologue de description pour le chercheur hollandais contre contributions spontanées ici) à moins qu'elle ne soit à mettre au compte d'une propriété de la langue en cause (néerlandais vs français québécois). Cependant, Fónagy et Fónagy (1983) semblent affirmer qu'une intonation légèrement montante à la fin d'une unité de parole est assez fréquente en discours oral, mais elle serait reliée à l'expressivité du locuteur.

Tableau 6.2 Répartition des schémas intonatifs des voyelles antépénultièmes, pénultidmes et finales des énoncés intratour dans les trois catégories

\begin{tabular}{|c|c|c|c|c|c|c|c|c|c|c|}
\hline & \multicolumn{3}{|c|}{ montées } & \multicolumn{3}{|c|}{ descentes } & \multicolumn{3}{|c|}{ neutres } & \multirow[t]{2}{*}{ Total } \\
\hline & $\bar{Z}$ & 4 & -1 & & $A$ & & $\rightarrow$ & $\mapsto$ & $\rightarrow$ & \\
\hline H & 6 & 23 & 3 & 5 & 3 & 3 & 2 & 10 & 2 & 57 \\
\hline$\%$ & 10,5 & 40,4 & 5,3 & 8,8 & 5,3 & 5,3 & 3,5 & 17,5 & 3,5 & \\
\hline$F$ & 3 & 20 & 14 & 8 & 4 & 3 & 1 & 5 & 2 & 60 \\
\hline$\%$ & 5 & 33,3 & 23,3 & 13,3 & 6,7 & 5 & 1,7 & 8,3 & 3,3 & \\
\hline Global & 9 & 43 & 17 & 13 & 7 & 6 & 3 & 15 & 4 & 117 \\
\hline \multirow[t]{2}{*}{$\%$} & 7,7 & 36,8 & 14,5 & 11,1 & 6 & 5,1 & 2,6 & 12,8 & 3,4 & \\
\hline & \multicolumn{3}{|c|}{$59 \%$} & \multicolumn{3}{|c|}{$22,2 \%$} & \multicolumn{3}{|c|}{$18,8 \%$} & \\
\hline
\end{tabular}

Le Tableau 6.1 et le Tableau 6.2 montrent dans l'ensemble des résultats somme toute assez similaires. Le seul élément digne de mention serait sans doute la forte représentation du schéma descendant-montant $(\downarrow)$, mais cette prédominance se retrouve aussi bien dans les énoncés finals de tour ( 6 cas sur 15, soit 40\%) que dans les énoncés intratour ( 43 cas sur 117 , soit 36,8\%), ce qui lui enlève toute valeur distinctive. Cela mènerait donc à minimiser le rôle de l'intonation ou plus 
prédsément de mouvement intonatif local comme élément importanê pour signaler fa fin d'un tour de parole.

Cette absence d'éléments intonatifs distinctifs nous pousse à croire que lindice de malité que nous recherchons ne se trouve pas au niveau local, c'est-à-dire pour les contours intonatifs des voyelles finales de tour. Mais avant de conclure ainsi, il conviendrait de s'assurer que la différence ne tiendrait pas à l'ampleur de la variation plutôt qu'à la forme du schéma intonatif. La finalité étant souvent associée dans la littérature à une intonation descendante, on pourrait par exemple penser que les fins de tour pourraient être marquées par une descente plus prononcée que les fins d'énoncé. Le Tableau 7 montre quîl n'en est rien, limportance du mouvement étant comparable en pourcentage.

Tableau 7: Moyenne de variation intonative (en $\mathrm{Hz}$ et en \%) entre les voyelles pénultèmes et finales des tours à schéma intonatfi descendant en comparaison avec la moyenne de varlation intonative des voyelles pénultièmes et finales des énoncés intratour schéma intonatif descendant

\begin{tabular}{c|c|c} 
moyennes & $\begin{array}{c}\text { voyelles pénultiémes et } \\
\text { finales des tours }\end{array}$ & $\begin{array}{c}\text { voyelles pénultièmes et } \\
\text { finales des énoncés } \\
\text { intratour }\end{array}$ \\
\hline$H z$ & $32,3 \mathrm{~Hz}$ & $41 \mathrm{~Hz}$ \\
\hline$\%$ & $24,3 \%$ & $26,85 \%$ \\
\hline
\end{tabular}

Par ailleurs, Ihypothèse de Fontaney (1987) voulant que les propositions affirmatives puissent avoir un mouvement descendant qui commence plus haut pour finir à un niveau « au-dessus du ton bas de repère » ce qui pourrait venir atténuer ou modifier « le message de finalité », n’a cependant pu être vérifié ici étant donné la quantité de données prises pour les voyelles cibles de chaque énoncé de notre corpus. Un simple regard sur le Tableau des données de fréquence à I'ANNEXE $B$ nous incite à penser que les valeurs de FO finale ne constituent pas un critère distinctif entre les fins de tour et les énoncés intratour. 


\subsection{La comparaison en demi-tons de la fréquence des voyelles finales et pénultièmes}

Une demière précaution nous amène à mesurer en demi-tons l'ampleur des mouvements intonatifs à la fin des tours et à la fin des énoncés intratour pour tenir compte d'éventuelles différences de « registre moyen » découlant de la déclinaison déjà observée. Les résultats présentés au Tableau 7.1 laissent voir une remarquable proximité des résuitats. Les énoncés descendants présentent une différence de 6,29 demi-tons en fin de tour contre 6,3 demi-tons pour les énoncés intratour alors que pour les énoncés montants, l'ampleur de variation est respectivement de 4,64 et de 4,78 demi-tons.

Tableau 7.1: Valeur moyenne de variation en demi-tons ${ }^{7}$ des voyelles pénultièmes et finales des énoncés intratour et des énoncés finals de tour pour les énoncés descendants et pour les énoncés montants

\begin{tabular}{c|c|c}
\hline & $\begin{array}{c}\text { voyelles pénultièmes et } \\
\text { finales des tours }\end{array}$ & $\begin{array}{c}\text { voyelles pénultièmes et } \\
\text { finales des énoncés } \\
\text { intratour }\end{array}$ \\
\hline Énoncés descendants & $-6,29 \mathrm{dt}$ & $-6,36 \mathrm{dt}$ \\
\hline Énoncés montants & $4,64 \mathrm{dt}$ & $4,78 \mathrm{dt}$ \\
\hline
\end{tabular}

Ces résultats mis en parallèle nous permettent cependant de constater une différence quant au comportement des énoncés descendants et des énoncés montants, peu importe leur situation dans le tour. En effet, on peut remarquer au Tableau 7.1 que les énoncés descendants provoquent une plus grande variation de Fo que les énoncés montants. On remarque une différence moyenne de l'ordre de 2 demi-tons en faveur des énoncés descendants. La quantité d'énoncés impliquée ne nous permet cependant pas d'extrapoler ces résultats sur une grande échelle.

7 * Calcul en demi-ton selon la formule : $\mathrm{dt}=40 \mathrm{X}\left(\log _{10}\right.$ (fr. finale/fr. pénultième). 


\subsubsection{SOMMAIRE DES OBSERVATIONS SUR L'INTONATION}

En bref, lanalyse de lintonation, divisée en trois grandes parties, nous a permis de constater certaines choses. Tout d'abord, lors de l'analyse des indices globaux, nous avons pu en quelque sorte reconnaître la présence d'une certaine ligne de déclinaison dans notre corpus avec $65 \%$ des valeurs des voyelles finales de tour qui sont inférieures aux valeurs moyennes de fo de leur tour respectif. En comparant la moyenne de FO des voyelles cibles de l'énoncé final avec la moyenne pour les autres énoncés du tour, nous avons également obtenu des résultats dans le même sens quoique moins bien définis. En effet, $50 \%$ des énoncés finals font voir une moyenne de fo pour les voyelles cibles inférieure à celles du tour alors que les valeurs étaient relativement égales dans $45 \%$ des cas, ce qui ne laisse que $5 \%$ pour les cas où l'énoncé final présente des valeurs supérieures.

Pour ce qui est de l'analyse des indices sectoriels, les résultats étaient beaucoup moins tranchés. En effet, l'utilisation des mêmes schémas intonatifs dans les deux situations présentes dans notre corpus, soit en fin de tour et intratour, rend difficile linterprétation des mouvements. Le même patron intonatif peut servir à deux intentions différentes comme c'est souvent le cas par exemple avec l'intonation montante qul peut être une question directe ou bien un moyen de conserver son tour de parole.

Dans notre corpus, un patron intonatif a retenu plus particulièrement notre attention. Il s'agit de la courbe descendante-montante ( $)$. Dans les deux situations étudiées, ce type de schémas était nettement plus fréquent que tous les autres. Si on se réfère au Tableau 6.1 et au Tableau 6.2, on remarque que ce schéma est produit dans $40 \%$ des énoncés finals et dans $36,8 \%$ des énoncés intratour. Ces résultats ne rejoignent pas du tout les résultats obtenus par Swerts et Collier (1992) et Swerts, Collier et Terken (1994) lors de leurs recherches où les 
intonations descendante-montantes étalent généralement perçues comme non finales ou au mieux pré-finales. D'allleurs, les intonations montantes, tout schéma confondu, représentent dans les deux situations plus de la moitté des énoncés, ce qui ne semblait pas être le cas pour ces deux études.

\subsubsection{L'intensité}

Après l'étude de la durée des pauses et celle de la fréquence fondamentale des tours, des énoncés et des voyelles finales, nous en venons maintenant à l'étude de IIntensité. Cet élément de notre recherche ne peut faire l'objet d'une analyse détallée étant donnée la complexité du paramètre d'intensité en lui-même et des caractéristiques de notre corpus. Ce dernier a été enregistré en laboratoire, certes, mais n'a pas été enregistré dans les conditions rigoureuses qui permettent une analyse contrôlée de l'énergie. Les locuteurs, qui étaient en conversation semidirigée, étaient relativement libres de bouger par rapport au microphone. Le son n'est alors pas constant et les valeurs d'intensité dont nous disposons sont celles qul ont été captées par le microphone et non celles qui ont été réellement produites par les locuteurs.

\subsubsection{LE TYPE D'ANALYSE}

Nous savons également que ce paramètre, encore plus que les autres, est très influencé par la nature même de la voyelle prononcée ainsi que par sa durée. Encore une fois, les conditions d'enregistrement ne permettent pas de vérifier ces valeurs de façon précise. De plus, de par la nature de notre recherche, cette précision ne s'avère pas essentielle. Dans ces conditions, les analyses effectuées dans cette partie seront faites sur une base très générale et les résultats obtenus devront donc être considérés avec circonspection comme ayant simplement une valeur indicative. 
Nous souhaitons aborder le phénomène de lintensité dans notre recherche d'une façon plutôt globale. Nous proposons alors une analyse comparable à la descente de fréquence que nous avons faite préalablement, en cherchant à vérifier s'll existe une descente générale de lintensité tout au long du tour de parole, descente qui serait parallèle à la déclinaison observée pour la fréquence.

\subsubsection{L'INTENSTTÉ EN DISCOURS SPONTANÉ}

Les recherches effectuées sur lintensité dans un contexte de discours spontané sont assez rares. Étant donné son caractère particulier, lintensité est le plus souvent étudiée dans un contexte très contrôlé. Les principaux travaux sur lesquels se base notre recherche sur le discours spontané, notamment ceux de Swerts (Swerts, 1993, 1997, 1998 ; Swerts et Collier, 1992 ; Swerts, Collier et Terken, 1994 ; Swerts, Bouwhuis, Collier, 1994 et Swerts et Geluykens 1994), ne parlent généralement pas de l'intensité. Quoique difficile à manipuler, ce paramètre a été tout de même abordé en spontané à l'échelle de l'énoncé par certains chercheurs, notamment par Laver (1994); certains travaux de l'équipe de Conrad Ouellon touchent aussi à cet aspect de la prosodie du discours spontané en français québécois.

Dans son ouvrage intitulé Principles of phonetics, Laver (1994: 505) décrit de façon assez simple le comportement de lintensité dans l'énoncé spontané en faisant ressortir explicitement une certaine similarité avec la ligne de déclinaison de lintonation :

Beyond the syllable, the linguistic use of loudness as a prosodic feature is comparable to that of pitch in some limited respects. As with pitch, it is reasonable to think of an utterance as having a loudness contour. In most types of utterances in most languages, the loudness declination not unlike that of pitch-declination, with a corresponding loudness baseline and topline. 
Il signale en outre que laugmentation dintensité a une fonction paralinguistique dans les situations de compétition pour prendre ou garder le tour de parole (Laver, 1994 : 506). Dans leurs études sur la perception des frontières d'énoncé en français québécois spontané, Ouellon (1990; 1991), Ouellon, Paradis et Duchesne (1991) et Duchesne (1991), observent une perte d'énergie importante de la syllabe finale par rapport à la syllabe qui a la plus haute valeur d'énergie dans l'énoncé pour les fins d'énoncé qui sont les mieux perçues. Ouellon (1991: 71) parle même directement de courbe d'énergie à l'intérieur de l'énoncé, mais sans envisager directement question de l'énergle dans le tour de parole.

\subsection{L'utilisation de lintensité dans le tour de parole}

Exploitant cette idée d'une descente de l'énergie en cours d'énoncé, nous avons donc cherché à voir si une pareille descente globale pouvait être observée du côté de l'unité plus grande que constitue le tour de parole. Une telle hypothèse n'est pas sans fondement physiologique, puisque l'on sait que c'est la pression d'air expulsé qui est responsable de l'intensité du son, cette pression diminuant au fur et à mesure que l'émission s'allonge; s'il ne fait pas de doute que la production d'un tour de parole s'accompagne de multiples inspirations qui constituent autant de « réinitialisations » potentielles de l'énergie, on peut néanmoins penser que cette capacité de réinitialisation diminue au fur et à mesure que le tour de parole s'allonge. De cette façon, la baisse graduelle de lintensité pourrait constituer un indice global que le tour de parole progresse et par conséquent approche de sa fin. C'est pour vérifier cette hypothèse que nous allons examiner divers aspects du comportement de l'intensité à lintérieur du tour de parole. 


\subsection{Lintensité moyenne}

Dans la perspective d'une descente générale de lintensité déjà signalée par les chercheurs et parallèle à la déclinaison de fréquence, nous avons voulu vérifier si lintensité moyenne des quatre voyelles cibles de l'énoncé final du tour était inférieure à l'intensité moyenne des quatre voyelles cibles de chacun des énoncés du tour pris dans leur ensemble. Le Tableau 7 nous montre les résultats des moyennes des valeurs d'intensité des énoncés finals par rapport à la moyenne d'intensité du tour.

Tableau 8 : Moyenne d'intensíté (en dB) de l'énoncé final ( 4 voyelles cibles) par rapport à la moyenne d'intensfté des tours sans la moyenne de l'énonos final ( 4 voyelles cibles par énoncé)

\begin{tabular}{c|c|c|c|c|c|c}
\hline $\begin{array}{c}\text { Tour de } \\
\text { parole }\end{array}$ & $\begin{array}{c}\text { Moy. dB } \\
\text { tour }\end{array}$ & $\begin{array}{c}\text { Moy. dB } \\
\text { énoncé }\end{array}$ & $\begin{array}{c}\text { Tour de } \\
\text { parole }\end{array}$ & $\begin{array}{c}\text { Moy. dB } \\
\text { tour }\end{array}$ & $\begin{array}{c}\text { Moy. dB } \\
\text { énoncé }\end{array}$ \\
\hline H-01 & 67,7 & $65,21^{8}$ & F-01 & 67,57 & 60,98 \\
\hline$H-02$ & 69,22 & 64,55 & F-02 & 67,82 & 71,23 \\
\hline$H-03$ & 70,87 & 66,14 & $F-03$ & 66,66 & 69,36 \\
\hline$H-04$ & 67,07 & 66,29 & $F-04$ & 63,55 & 59,16 \\
\hline$H-05$ & 68,31 & 66 & $F-05$ & 64,59 & 60,7 \\
\hline$H-06$ & 66,19 & 60,33 & $F-06$ & 66,4 & 62,32 \\
\hline$H-07$ & 66,19 & 65,66 & $F-07$ & 63,71 & 60,54 \\
\hline$H-08$ & 62,73 & 57,93 & $F-08$ & 69,9 & 70,14 \\
\hline$H-09$ & 63,31 & 66,53 & $F-09$ & 69,08 & 67,65 \\
\hline$H-10$ & 62,4 & 62,81 & $F-10$ & 68,68 & 75,46 \\
\hline MOYENNE & 66,4 & 64,15 & & 66,80 & 65,75 \\
\hline
\end{tabular}

D'une manière générale, 14 tours sur $20(70 \%)$ présentent une valeur moyenne pour l'énoncé final inférieure à la moyenne d'intensité du tour. Cette variation allant de $-0,53 \mathrm{~dB}$ à $-6,59 \mathrm{~dB}$. On retrouve même dans 7 énoncés finals sur les 14

${ }^{8}$ En gras, les cas où la valeur moyenne de lintensité de l'énoncé final est inférieure à la moyenne du tour. 
qui ont une valeur moyenne d'intensité inférieure à la valeur moyenne de leur tour respectif, une différence d'au moins $4 \mathrm{~dB}$, ce qui est relativement important. Cela veut dire que dans le nombre de tours pour lequel l'énoncé final affiche une énergie sensiblement plus basse que la moyenne du tour, $50 \%$ affichent une valeur nettement inférieure. Par contre, si l'on revient sur les 6 cas où lintensité de l'énoncé final est supérieure à l'intensité moyenne du tour, on constate qu'il n'y en a qu'un pour lequel la différence est supérieure à $4 \mathrm{~dB}$, soit le tour $\mathrm{F}-10$ avec une variation de $+6,78 \mathrm{~dB}$. Les locuteurs de notre corpus montrent donc bien une certaine tendance à produire des énoncés de fin de tour avec une intensité moyenne moins élevée que l'intensité moyenne du tour.

\subsection{La ligne d'intensité globale}

Il apparaît alors intéressant de vérifier l'orientation de la ligne d'intensité globale à partir des valeurs d'intensité produites par les locuteurs pour chacun de leur tour. Le Graphique 1 présente pour chacun des 20 tours de parole la droite de régression obtenue à partir des valeurs d'intensité en $\mathrm{dB}$ des 4 voyelles cibles. Ce graphique nous permet donc de visualiser l'existence d'une orientation globale des valeurs d'intensité dans le tour de parole. Compte tenu des propriétés des valeurs des $X$, l'utilisation de la droite de régression et des valeurs de pente a ici une fonction illustrative plutôt que strictement statistique. 
Graphique 1: Declinaison dintensité des tours de parcle de th th avec valeur de pente (m) et coefficient de détermination $\left(R^{2}\right)$

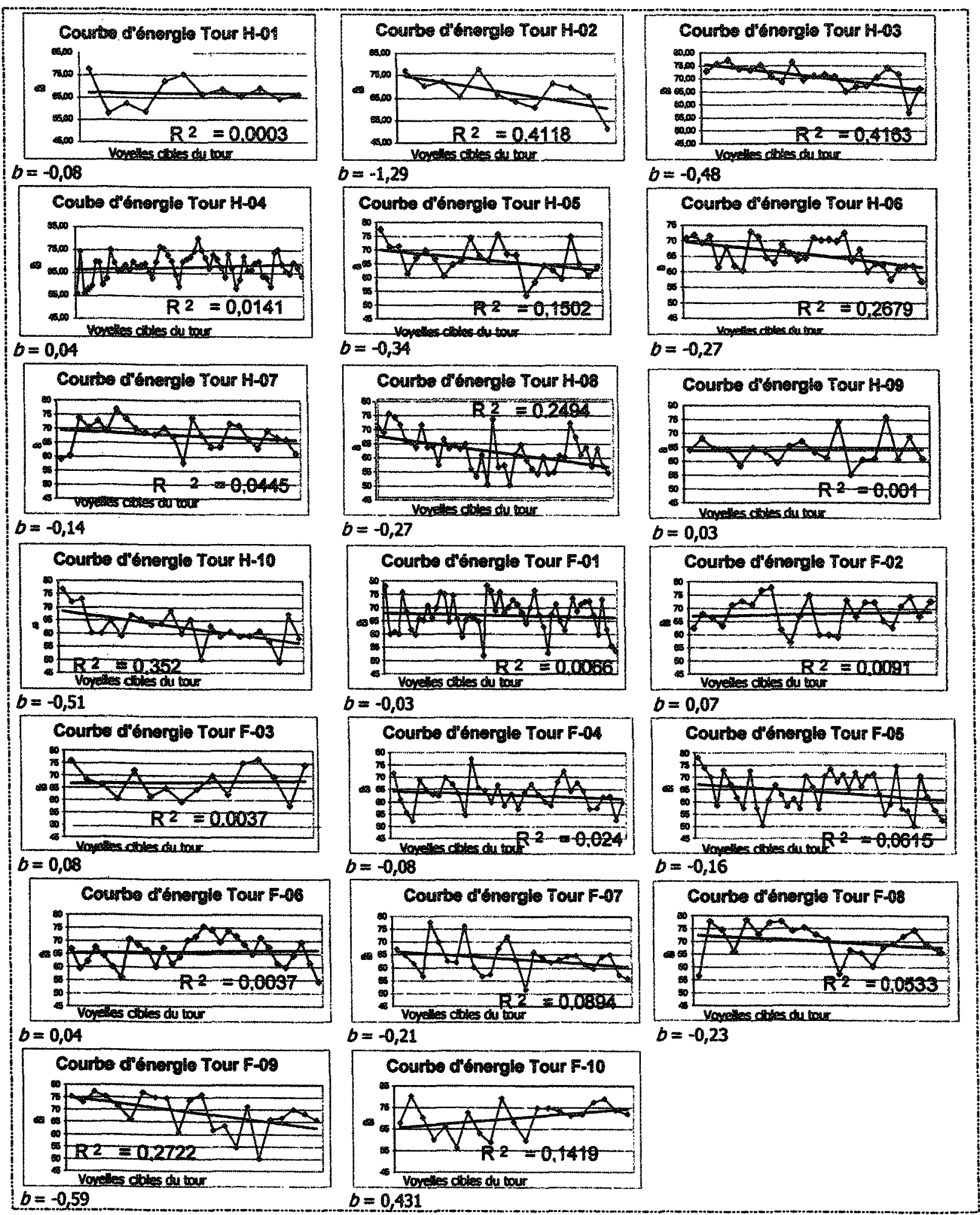


L'examen des valeurs de pente (b) permet de considérer que 9 des 20 tours (H-02, $\mathrm{H}-03, \mathrm{H}-05, \mathrm{H}-06, \mathrm{H}-08, \mathrm{H}-10, \mathrm{~F}-07, \mathrm{~F}-08$ et F-09) présentent une courbe d'énergle nettement descendante $(b<-0,2)$, ce qui représente $45 \%$ des tours étudiés. De plus, deux tours ( $\mathrm{H}-07$ et $\mathrm{F}-05$ ) affichent une pente légèrement descendante avec une valeur de $(b)$ se situant entre $-0,1$ et $-0,2$. Si on ajoute ces deux tours aux premiers, il y a alors une proportion de $55 \%$ des tours du corpus (11 tours sur 20) qui présentent une ligne d'énergie descendante, ce quil n'est vraiment pas à négliger. En contrepartie, il n'y a qu'un tour $(F-10)$ qui montrent une énergie franchement montante avec une valeur de $(b)$ de 0,43 . Enfin, un total de 8 tours (H-01, H-04, H-09, F-01, F-02, F-03, F-04, F-06) peuvent être considérés comme ne laissant pas voir d'orientation précise avec une valeur de pente se situant en $-0,1$ et $+0,1$. Il semblerait alors, à la lumière de ces résultats, que lídée de déclinaison de la ligne dîntensité que propose Laver (1994) pour l'énoncé, par comparaison avec la déctinaison de la ligne de fréquence, se retrouverait, aussi, d'une certaine manière, à un niveau plus grand que constitue le tour de parole.

En regardant ce graphique, on se rend en outre compte qu'il y a une différence en quelque sorte qualitative entre les lignes de tendance descendantes et les lignes montantes. On peut remarquer qu'il y a beaucoup de lignes de tendance franchement descendantes. On en compte facilement neuf contre seulement une quil est franchement montante ( $F-10)$. De ces neuf, il $y$ en a 6 qui ont un coefficient de détermination $\left(R^{2}\right)$ de plus de 0,2 , ce qui indique que les valeurs individuelles ne sont tout de même pas trop dispersées par rapport à la droite de régression, celle-ci donnant une assez bonne représentation de la tendance générale descendante de ces tours. Par contre, aucune droite montante, pas même $\mathrm{H}-10$, ne dépasse ce seuil de 0,2 , les valeurs étant plus dispersées et la ligne de tendance apparaissant moins représentative des valeurs réelles du tour. 


\subsection{L'intensité de la voyelle finale}

Après avoir constaté la présence d'une certaine descente de lintensité dans le tour avec la valeur moyenne d'intensité de l'énoncé final par rapport à la moyenne de son tour ainsi que la présence assez importante d'une ligne d'intensité globale généralement descendante dans les tours de notre corpus, il serait intéressant de se demander comment se situe la valeur d'intensité de la voyelle finale du tour par rapport à la moyenne du tour correspondant. Nous savons, à partir des travaux de Ouellon (1990; 1991), Ouellon, Paradis et Duchesne (1991) et Duchesne (1991), qu'on observe, en oral spontané québécois, une perte d'énergie importante de la syllabe finale par rapport à la syllabe qui a la plus haute valeur d'énergie dans l'énoncé. Nous cherchons ici à vérifier s'il existe une perte d'énergie comparable sur la voyelle finale de tour par rapport à la valeur d'intensité moyenne des autres voyelles cibles du tour de parole. Dans le Tableau 9, les valeurs d'intensité des voyelles finales de tour sont comparées avec les valeurs moyennes d'intensité des tours correspondants.

Tableau 9 : Valeur d'intensité (en $\mathrm{dB}$ ) de la voyelie finale de tour par rapport à la moyenne d'Intensitté des voyelles clbles des tours sans la moyenne de l'énoncé final (4 voyelles cibles par énoncé)

\begin{tabular}{c|c|c|c|c|c}
\hline $\begin{array}{c}\text { Tour de } \\
\text { parole }\end{array}$ & $\begin{array}{c}\text { Moy. dB } \\
\text { tour }\end{array}$ & $\begin{array}{c}\text { Val. en dB de } \\
\text { la voy. finale: }\end{array}$ & $\begin{array}{c}\text { Tour de } \\
\text { parole }\end{array}$ & $\begin{array}{c}\text { Moy. dB } \\
\text { tour }\end{array}$ & $\begin{array}{c}\text { Val. En dB de } \\
\text { la voy. finale }\end{array}$ \\
\hline$H-01$ & 67,7 & 66,28 & F-01 & 67,57 & 53,75 \\
\hline$H-02$ & 69,22 & 51,26 & $F-02$ & 67,82 & 72,73 \\
\hline$H-03$ & 70,87 & 66,14 & $F-03$ & 66,66 & 74,20 \\
\hline$H-04$ & 67,07 & 63,52 & $F-04$ & 63,55 & 59,71 \\
\hline$H-05$ & 68,31 & 63,87 & $F-05$ & 64,59 & 52,72 \\
\hline$H-06$ & 66,19 & 56,84 & $F-06$ & 66,4 & 54,26 \\
\hline$H-07$ & 66,19 & 60,99 & $F-07$ & 63,71 & 55,67 \\
\hline$H-08$ & 62,73 & 54,64 & $F-08$ & 69,9 & 65,66 \\
\hline$H-09$ & 63,31 & 60,88 & $F-09$ & 69,08 & 65,60 \\
\hline$H-10$ & 62,4 & 58,32 & $F-10$ & 68,68 & 71,95 \\
\hline MOYENNE & 66,4 & 60,27 & & 66,80 & 62,63 \\
\hline
\end{tabular}

${ }^{9}$ En gras, les cas où la valeur moyenne de l'intensité de l'énoncé final est inférieure à la moyenne du tour. 
On peut constater que 17 voyelles finales sur $20(85 \%)$ ont une valeur d'intensité inférieure à la valeur moyenne des voyelles cibles de leur tour correspondant. Les valeurs moyennes des voyelles finales de tour se situent à $60,27 \mathrm{~dB}$ chez $\mathrm{H}$ et à $58,20 \mathrm{~dB}$ chez $F$, comparativement à des valeurs dintensité moyennes de tour de $66,4 \mathrm{~dB}$ pour $\mathrm{H}$ et de $66,80 \mathrm{~dB}$ pour $F_{\text {. }}$ On peut donc remarquer une variation moyenne de plus de $6 \mathrm{~dB}$. Ces résultats nous portent à croire que, pour notre corpus, indépendamment de l'orientation de la courbe d'intensité finale, montante ou descendante, l'énergle de la voyelle finale aurait tendance à être plus basse que l'énergle moyenne du tour; cela suggère la possibilité que le locuteur pourrait chercher à produire un effet de finalité en utilisant une valeur d'intensité nettement inférieure aux valeurs d'intensité utilisées dans son tour.

Avant d'accréditer une telle hypothèse, il serait bon, sachant que la voyelle finale d'énoncé a déjà tendance à être moins forte (Ouellon, 1990 et 1991 ; Ouellon, Paradis et Duchesne, 1991 ; Duchesne, 1991) de s'assurer que la voyelle finale de tour se distingue des autres voyelles finales d'énoncé par une intensité encore plus faible, sans quoi il est difficile d'y voir un indice de finalité de tour. Le Tableau 9.1 nous montre les résultats de cette vérification. 
Tableas 9.1: Comparalson des valeurs dintensite (en dis) de la voyelle male de cost

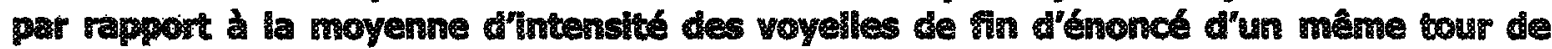
sarole

\begin{tabular}{c|c|c|c|c|c}
$\begin{array}{c}\text { Tour de } \\
\text { parole }\end{array}$ & $\begin{array}{c}\text { Moy. dB des } \\
\text { voyelles de } \\
\text { fin d'énoncé }\end{array}$ & $\begin{array}{c}\text { Val. en dB } \\
\text { de la voy } \\
\text { fnale }\end{array}$ & $\begin{array}{c}\text { Tour de } \\
\text { parole }\end{array}$ & $\begin{array}{c}\text { voy. dB delles de } \\
\text { fin d'énoncé }\end{array}$ & $\begin{array}{c}\text { Val. en dB } \\
\text { de la voy. } \\
\text { finale }\end{array}$ \\
\hline$H-01$ & 64,64 & 66,28 & F-01 & 63,37 & 53,75 \\
\hline$H-02$ & 63,22 & 51,26 & $F-02$ & 65,77 & 72,73 \\
\hline$H-03$ & 67,57 & 66,14 & $F-03$ & 64,96 & 74,20 \\
\hline$H-04$ & 64,04 & 63,52 & $F-04$ & 58,37 & 59,71 \\
\hline$H-05$ & 60,16 & 63,87 & $F-05$ & 60,65 & 52,72 \\
\hline$H-06$ & 63,69 & 56,84 & $F-06$ & 63,2 & 54,26 \\
\hline$H-07$ & 62,97 & 60,99 & $F-07$ & 58,28 & 55,67 \\
\hline$H-08$ & 59,37 & 54,64 & $F-08$ & 68,69 & 65,66 \\
\hline$H-09$ & 61,31 & 60,88 & $F-09$ & 65,87 & 65,60 \\
\hline$H-10$ & 56,8 & 58,32 & $F-10$ & 67,77 & 71,95 \\
\hline
\end{tabular}

On observe dans un premier temps que la voyelle de fin de tour fait voir dans 13 cas sur 20 une intensité inférieure à l'intensité moyenne des voyelles de fin d'énoncé, ce qui est en accord avec la tendance générale à la baisse d'intensité. II faut cependant éviter de conclure trop vite qu'il s'agit d'un indice fiable de fin de tour. Dans plusieurs cas, l'écart d'intensité est trop faible pour être considéré comme perceptible. Si l'on prend un seuil de $4 \mathrm{~dB}$ par exemple, seulement 6 voyelles finales de tour sur 20 sont encore plus basses que la valeur moyenne d'intensité des voyelles de fin d'énoncé. Il faut en outre considérer que la comparaison se fait avec la moyenne des voyelles de fin d'énoncé, ce qui n'exclut pas qu'll puisse se trouver à l'intérieur du tour une fin d'énoncé dont l'intensité est plus faible que la fin de tour, et qui pourrait légitimement être interprétée comme une fin de tour selon ce critère. Effectivement, en consultant I'ANNEXE $C$ on voit qu'll n'y a que trois tours ( $\mathrm{H}-02, \mathrm{H}-06$ et $\mathrm{F}-06)$, pour lesquels la voyelle finale de tour soit effectivement la moins intense de toutes les voyelles finales. Dans ces conditions, on peut difficilement soutenir que la plus faible intensité de la voyelle finale de fin de tour est un indice direct et déterminant de fin de tour de parole ; cela ne signifie toutefois pas qu'on ne peut considérer l'abaissement d'intensité 
comme susceptible de s'ajouter à d'autres indices pour favoriser une interprétation de finalité.

En résumé, lidentification des mouvements d'intensité dans notre corpus a permis de constater une tendance à l'utilisation d'une énergie plus faible à la fin des unités. À cet égard, le Tableau 8 a montré que les deux locuteurs du corpus avaient une tendance à produire l'énoncé final de tour avec une moyenne d'intensité plus basse que la moyenne de l'ensemble des énoncés du tour correspondant. Dans une proportion de $70 \%$, les énoncés finals présentaient une énergie inférieure à celle du tour, la différence étant supérieure à $4 \mathrm{~dB}$ dans la moitié d'entre eux. Le graphique 1 a permis quant à lui de vérifier l'existence d'une certaine ligne de déclinaison globale dintensité dans les tours de parole de notre corpus. Non seulement l'orientation descendante est nettement plus fréquente que l'orientation montante, mais elle est aussi en général plus prononcée et plus révélatrice de l'ensemble des valeurs du tour.

Le Tableau 9 nous a montré que la voyelle de fin de tour affichait dans $85 \%$ des cas une valeur d'intensité inférieure à la moyenne d'intensité du tour. Ce résultat doit cependant être nuancé si on tient compte de la faiblesse de la différence dans certains cas et surtout du fait qu'une telle tendance à une baisse de lintensité existe aussi pour toutes les voyelles finales d'énoncé.

L'examen du fonctionnement de lintensité dans notre corpus ne permet donc pas de dégager un indice direct qui agirait auprès de l'interlocuteur comme un signal explicite et non équivoque de fin de tour, et cela, même si notre analyse a confirmé l'existence d'une tendance générale à une baisse de l'énergie au fur et à mesure que l'on s'approche de la fin du tour. 


\subsection{LES IRDICES NON PROSODIQUES}

Nous devons maintenant regarder du côté des facteurs non prosodiques afin de voir ce que ce type d'indices peut nous apporter dans l'explication des changements de tour de parole. Cette section s'intéresse donc au rôle de certains éléments linguistiques, autres que les facteurs prosodiques déjà étudiés, comme le rôle des facteurs syntaxiques et sémantico-pragmatiques, rôle déjà suggéré par Cutler et Pearson (1986: 139) dans leur analyse des indices prosodiques des tours de parole :

To take over the turn at the appropriate moment, without undue hesitation, it is obviously useful to be able to decide as early as possible that the previous speaker has finished or is about to finish. Clearly, syntax, semantics and reference to the discourse context play the largest role here. A completed utterance usually signal that a response is required. Anecdotes have ends (if not always punch lines). And there are many more text-internal cues to whether or not a speaker has finished.

Les éléments que nous avons choisis de relever sont donc inspirés de ceux identifiés par Cutter et Pearson (1986) que nous avons regroupés par catégories. En ce qui concerne la syntaxe, trois aspects ont été considérés, c'est-à-dire le type de phrases, la complétion grammaticale ainsi que la longueur. Du point de vue des facteurs sémantico-pragmatiques, les indices de conclusion, de non-conclusion, de jugement de valeur, de redondance, les mots clé et les hésitations ont été relevés.

\subsubsection{Les indices syntaxiques}

Nous appuyant sur les résultats des études antérieures sur le rôle des facteurs syntaxiques dans l'annonce d'un changement de tour de parole (Duncan, 1972 ; Cutler et Pearson, 1986), il nous a semblé intéressant et pertinent d'examiner en 
premier lieu les types d'énoncés ${ }^{10}$ produits par les locuteurs autant à lintérieur des tours qu'à la fin. Nous avons inclus dans notre analyse, à la fin de chaque énoncé, la notation $D$ pour déclaratif, I pour interrogatif et $E$ pour exclamatif (voir ANNEXE A). Les résultats obtenus, regroupés dans le Tableau 10, nous ont rapidement montré que la très grande majorité des énoncés du corpus sont de type déclaratif autant pour les finals que pour les énoncés intratour ( $80 \%$ chez $\mathrm{H}$ et $90 \%$ chez $F$ pour les énoncés finals; $96,8 \%$ et $91 \%$ dans le cas des énoncés non finals). Il n'y a donc rien de concluant sur ce plan sauf peut-être pour dire que les locuteurs ont tendance à produire un discours énonciatif, ce qui n'est pas vraiment surprenant étant donné le type de discours représenté dans notre corpus.

Tableau 10: Répartítion des énonoés du corpus pour chacun des locuteurs par leur position dans le discours et selon qu'ilis sont déclaratif, interrogatif ou exclamatif

\begin{tabular}{l|c|c|c|c} 
& Locuteurs & Déclaratif & Interrogatif & Exclamatif* \\
\hline $\begin{array}{l}\text { Enoncés } \\
\text { intratour }\end{array}$ & $H(62)^{* *}$ & $96,8 \%(60)$ & $1,6 \%(1)$ & $1,6 \%(1)$ \\
\hline Enoncés & $F(67)$ & $91 \%(61)$ & $6 \%(4)$ & $3 \%(2)$ \\
finals & $H(10)$ & $80 \%(8)$ & - & $20 \%(2)$ \\
\hline
\end{tabular}

*Le type impératif n'est pas inclus dans le tableau puisqu'il n'est pas représenté dans le corpus.

**Le nombre d'énoncés intratour pour $H$ est normalement de 64. Les deux énoncés manquants (H-03-01 et H04-01) n'ont pas été notés parce quills présentaient des contenus très incomplets ce qui rendait hasardeux de leur apposer une étiquelte adéquate.

\subsubsection{LA COMPLÉTION}

Dans un deuxième temps, nous avons jeté un œil du côté de la complétion des phrases. Il nous fallait vérifier sill y avait, encore là, une différence entre les énoncés intratour et les énoncés finals. Rappelons que Duncan (1972) inclut la complétion grammaticale dans ses « six signes de changement de tour de parole » sous la rubrique syntaxe (p. 286-287). Cutler et Pearson (1986) signalent par

${ }^{10}$ Les énoncés ne doivent pas être confondus avec les notions de phrase et de proposition. Se référer à la méthodologie dans la section 3.2.5 La délimitation des énoncés. 
contre que la complétion syntaxique d'une proposition à elle seule ne semble pas être un indice de changement de tour très efficace, car linterlocuteur tenterait de prendre la parole à chaque fin de phrase alors que le tour n'est pas encore cédé (p. 140). Tous semblent cependant affirmer que la complétion n'est un indice valable que si elle est accompagnée par d'autres indices de finalité. Cela semble nettement le cas dans notre corpus où la complétude grammaticale se retrouve dans la majorité des énoncés, finals comme non finals et ne saurait donc constituer un signal valable de fin de tour. Après vérification, II semblerait qu'il n'existe aucune différence entre les énoncés intratour et les énoncés finals, la grande majorité des énoncés étant complets.

Sur les 151 énoncés du corpus, on n'en retrouve que 8 qui ne sont pas complétés. De ce nombre, 5 sont des énoncés finals contre 3 qui sont des énoncés intratour. Ce qui peut être intéressant de remarquer ici, c'est que sur les 8 énoncés non complétés du corpus, 6 sont produits par le locuteur $F$ et, de ces 6,4 sont des énoncés finals (F-01,-02,-03,-08). Ce que ces tours ont en commun, C'est qu'ils sont assez longs (F-01: 73 sec., F-02 : 36 sec., F-03 :29 sec., F-08: 37sec.), mais surtout ils sont marqués par une forte présence d'hésitations tout au long du tour, hésitations qui deviennent même plus présentes dans la dernière partie. Il en va de même pour le tour de $\mathrm{H}(\mathrm{H}-09)$ qui se termine sur une hésitation. Ces tours sont terminés abruptement par une coupure de la parole par linterlocuteur. L'énoncé final du tour F-01 en est un bon exemple :

F-01-14: * Là tu fais les côtes d'Alaska etc., etc. (resp.) pis euh tu fais quoi donc, tu j'pense que bon y doit y avoir des escales un moment donné là bon ben tu vois euh... »

H a \& Qu'est-ce qu'y a à voir là ? Y a pas grand-chose! » 
Les hésitations de ce segment ne se retrouvent pas uniquement dans les « euh » émis par le locuteur, mais aussi dans la façon dont le propos est dit. Le discours ressemble à celui de quelqu'un qui réfféchit à voix haute.

Cela nous porte à croire, tout de même avec une certaine prudence, que lorsque le tour est en cours depuis un certain temps déjà et que le locuteur semble hésiter dans ses propos, il y a de fortes chances que linterlocuteur intervienne dans le discours solt en complétant les paroles du locuteur, soit en orientant la conversation par une question servant à relancer le discours sur un sujet connexe. Dans l'ensemble des tours de parole, le locuteur $F$ produit des tours de parole qui sont en moyenne plus longs que $H$ et les tours de $F$ sont plus souvent ponctués d'hésitations que les tours de $H$.

\subsubsection{LA LONGUEUR}

Nous avons enfin abordé le rôle de la dimension syntaxique sous un autre angle, un angle plus quantitatif, en cherchant à voir s'il existait un lien entre la position dans le tour et la longueur des énoncés. Dans l'analyse du corpus de leur étude sur linteraction homme-machine, Swerts et Ostendorf (1997: 33) ont remarqué une tendance à produire des énoncés plus longs en début de discours, ce qui permettait au locuteur de diminuer la longueur des énoncés suivants en omettant les informations redondantes.

Du côté de notre analyse, il faut tout d'abord préciser que les énoncés du corpus ont été découpés à l'aide de l'enregistrement audio et de la transcription du corpus. Les frontières demeurent donc pour une part subjectives, et de ce fait discutables, ce qui est souvent le cas lorsque l'on travaille avec du discours spontané. Ce fait étant établi, nous avons voulu tout de même chercher à vérifier ce phénomène de raccourcissement progressif observé par Swerts et Ostendorf 
(1997). Le Tableau 11 rous montre la longueur en syllabes des énoncés finals par rapport à la longueur moyenne des énoncés intratour. Si on regarde du côté du locuteur $H$, on observe une tendance très marquée à produire des énoncés plus courts en fin de tour de parole (7 cas sur 10), contrairement à ce qui se passe pour $F$ (4 cas sur 10$)$.

Tablean 11 : Longueur en syllabes orales des énoncés finals par rapport à la longueur moyenne en syllabes orales des énonoés intratour"

\begin{tabular}{l|c|c|c|c|c}
\hline & $\begin{array}{c}\text { Enonce } \\
\text { final }\end{array}$ & $\begin{array}{c}\text { Moy. } \\
\text { enoncés } \\
\text { intratour }\end{array}$ & $\begin{array}{c}\text { Enonce } \\
\text { final }\end{array}$ & $\begin{array}{c}\text { Moy. } \\
\text { enoncés } \\
\text { intratour }\end{array}$ \\
\hline$H-01$ & 4 & 37,3 & $F-01$ & 45 & 18,1 \\
\hline$H-02$ & 17 & 30 & $F-02$ & 12 & 23,8 \\
\hline$H-03$ & 3 & 13,6 & $F-03$ & 54 & 24,7 \\
\hline$H-04$ & 7 & 14,5 & $F-04$ & 6 & 35,1 \\
\hline$H-06$ & 34 & 13,4 & $F-05$ & 14 & 22,6 \\
\hline$H-07$ & 8 & 16,4 & $F-06$ & 59 & 38,1 \\
\hline$H-08$ & 12 & 10,9 & $F-07$ & 38 & 31,3 \\
\hline$H-09$ & 21 & 17,1 & $F-08$ & 65 & 20,6 \\
\hline Moyenne & 13 & $1 F-09$ & 16 & 18,6 \\
\hline & 13 & 17 & $F-10$ & 68 & 28,8 \\
\hline
\end{tabular}

D'un point de vue plus individuel, il est intéressant de regarder ce qui se passe du côté des tours eux-mêmes. Pour les tours $\mathrm{H}-01, \mathrm{H}-02, \mathrm{H}-03$ et $\mathrm{H}-10$, les énoncés finals sont réellement les plus courts de leur tour respectif; on peut aussi constater la nette tendance au raccourcissement des énoncés en finale pour les tours $\mathrm{H}-04$ et $\mathrm{H}-06$. Dans le cas des tours $\mathrm{H}-05$ et $\mathrm{H}-07$, qui ont des énoncés finals très longs, une analyse plus fine permet d'observer que ces énoncés anormalement longs interviennent en fait après une séquence qui laisse voir une certaine tendance au raccourcissement $(\mathrm{H}-05$ : 15-33-5-9-5-34 et $\mathrm{H}-07$ : 1-3-21-827-10-6-28), comme si I'on était en présence en quelque sorte d'une fausse fin ou d'une « erreur » sur laquelle venait se greffer un énoncé long.

${ }^{11}$ Les données utilisées pour ce tableau sont présentées à l'annexe $D$. 
Lorsqu'on considère par contre les résultats de F, il est évident qu'll est impossible dans ce cas de conclure que le raccourcissement des énoncés constitue un quelconque indice de finalité. Ce qui était très concluant pour le locuteur $\mathrm{H}$, ne l'est pas du tout pour F. On ne retrouve en effet que 4 tours sur 10 qui ont un énoncé înal plus court que la moyenne des énoncés intratour. En fait, F-04 est le seul tour où l'énoncé final est réellement le plus court du tour, même si les tours F-04, F-05 et F-07 peuvent laisser voir une certaine tendance au raccourcissement -bien relative compte tenu de la longueur des énoncés - à mesure que le tour progresse. (F-04: ...68-54-10-6, F-05: ...41-28-10-14 et F-07: ...76-40-38). On voit donc assez rapidement que les deux locuteurs ont des comportements discursifs très différents en ce qui concerne la distribution de la longueur de leurs énoncés à lintérieur du tour.

On ne peut donc pas conclure que ce facteur de raccourcissement progressif des énoncés est un indice fiable de finalité dans l'ensemble du corpus, seulement 11 tours sur 20 montrent une longueur en syllabes plus courte que celle de la moyenne des énoncés de leur tour respectif. Il est tout de même intéressant de souligner que ce facteur pourrait jouer un rôle comme indice de finalité chez un de nos locuteurs $(H)$, ce qui incite à croire que l'on pourrait être en présence de stratégies discursives individuelles, $\mathrm{F}$ s'exprimant au contraire avec des énoncés plus longs sans que l'on puisse observer de tendance au raccourcissement en fin de tour.

\subsubsection{Les indices sémantico-pragmatiques}

Les facteurs sémantico-pragmatiques fonctionnent de façon un peu différente des autres facteurs analysés dans notre étude et leur étude ne peut se limiter uniquement à l'énoncé final, puisque leur portée est en général beaucoup plus large. Nous avons donc ajusté notre méthode d'analyse pour ces facteurs. Le 
procédé que nous avons ưtilisé consiste à repérer les indices de non-conclusion, de conclusion, de redondance, de même que la présence de signaux explicites de finalité dans le tour de parole.

\subsubsection{LA PRÉSENTATION DES INDICES}

La principale difficulté rencontrée lors de lidentification des indices sémanticopragmatiques, c'est que les expressions qui les constituent ne sont pas cloisonnées à un type dindices en particulier. On peut, par exemple, rencontrer les mots « Ça fait que... » et leurs variantes en tant quindice de non-conclusion à un endroit et allleurs, ces mots représentent un indice de conclusion. Ces mots, utilisés après lintroduction d'un nouvel élément de la conversation, peuvent être perçus comme annonciateur d'une suite au récit, encore plus s'ils sont accompagnés d'une intonation montante ou quiils sont prononcés fortement. Sills sont prononcés à la toute fin du récit, et souvent prononcés très faiblement, ils seront compris comme une façon pour le locuteur d'annoncer qu'il a fait le tour du sujet et que le locuteur peut prendre la parole. 


\section{Exemple:}

H-88

F comment ca?

H-08-01 Non, non, jaurais... jaurals pu.

H-08-02 Ben, mon père enseignait là, ca m'aurait fait un lift (rires) mais autrement on s'était arrangés autrement.

H-08-03 Ma soeur allait déjà à Garneau, ma mère travaillait à Saint-Sacrement.

F $\quad$ o.K.

H-08-04 FAT QU'ON... j’ai dit : j'vais aller à Garneau, ca va être plus simple.

H-08-05 Pis on faisalt la run, j'faisais la run de lait, là, le matin. (INDICE DE NONCONCLUSION)

F $\quad 0 . K$

H-08-06 Déposer ma mère, après ça ma soeur pis le soir je reprenais ma soeur, ma mère souvent.

F Ah, oui.

H-08-07 ... en auto... mais en fait, c'est ça, c'est que finalement on avait une auto pour les trois qui se promenaient dans l'coin de Garneau/Saint-Sacrement.

H-08-08 Pis mon père avait l'autre auto.

$\boldsymbol{F}$

Ah.

H-08-09 FATT QUE C'EST CA. (INDICE DE CONCLUSION)

H-08-10 Mais si j’étais allé à Limoilou euh, j'connais quasiment tous les profs de français.

H-08-11 J'aurais eu euh, j'aurais eu du fun dans leurs cours.

F

*Hmm, hmm. Mais c'est difficile, hein, parce que dans un autre, dans un autre sens si tu réussis moins bien euh, tas plus de pression, hein.

Contrairement aux facteurs prosodiques qui sont quantifiables et pour lesquels il est possible d'appuyer nos résultats sur des valeurs numériques concrètes, les facteurs sémantico-pragmatiques ne se prêtent pas à l'analyse quantitative et dolvent être interprétés qualitativement. Ils sont étroitement reliés au contenu du discours et c'est avec cette perspective en tête que nous avons cherché à identifier et à classer ces indices. 


\subsection{Les indices de non-conclusion}

Pour comprendre la dynamique sémantico-pragmatique des tours de parole, il faut tenir compte de la présence d'indices de non-conclusion. Tout autant que les indices de conclusion, les indices de non-conclusion sont des mots ou des expressions plus ou moins explicites qui laissent entendre que le tour de parole en cours n'est pas fini et qu'il y a encore des éléments qui vont suivre. Certains de ces indices peuvent être très généraux alors que d'autres peuvent être plus spécifiques (on y retrouve même des questions rhétoriques). Un des éléments qui représente assez bien les indices de non-conclusion est le mot « là » accompagné d'une intonation montante $(. . . l a ̀+\%)$. On peut le retrouver dans des énoncés du genre de l'exemple qui suit et qui laissent réellement comprendre à linterlocuteur que d'autres informations vont venir.

\section{Exemple:} H-10-03 T'sais que si tu fais, si tu fais quelque chose qui est pas correct là $\pi$, ca passera
pas pantoute.

H-10-04 Par contre, tu sais que si tu fais quelque chose qui est pas correct, pis tu linscris, tu l'indiques, ça va être correct.

\subsection{Les indices de conclusion}

Les indices de conclusion sont généralement des indications qui nous font comprendre que la fin du récit approche. On peut les reconnaître par exemple sous la forme du dévoilement de la conclusion d'un récit, de l'élément clé de I'histoire en cours, de la morale ou de la «leçon» d'une intervention. Nous avons déjà mentionné en méthodologie l'exemple de la promesse faite en début de récit qui a été remplie et qui, ce faisant, marque en quelque sorte la fin du récit. 
Linterlocuteur peut alors penser, avec raison, que la parole est maintenant libre et quill peut parler à son tour:

\section{Exemple:}

...

H-01-02 T'sais, y brûlait ça avec euh, de l'azote liquide là, j'pense, pis elle avait dit : si tu pleures pas, j'vais todonner une gomme. (PROMESSE)

H-01-03 Pis effectivement, comme de falt, c'était à I'Hôtel-Dieu j'pense, à fin là, dans l'vieux Québec là, à la fin là, quand mes verrues avaient été brûlées, j'avais pas braillé, javais eu mes deux pottes commes Chicklets. (PROMESSE REMPLIE)

H-01-04 J'tais ben content. (Fin du tour)

Nous donnons, dans les sections qui suivent, quelques exemples des principales formes que peuvent prendre les indices de conclusion.

\subsection{Les indices de jugement de valeur}

Les indices de jugement de valeur prennent la forme de propos ou d'expressions utilisées par le locuteur luj-même pour évaluer son récit ou bien les faits quili vient d'énoncer. Le locuteur semble utiliser ces expressions lorsque, à la fin de son récit, il ne salt plus trop quoi ajouter.

\section{Exemple:}

...

H-10-05 T'sais, si tu remarques tes erreurs, si tu reconnais tes erreurs là, ca va être correct.

H-10-06 Sauf que tu y passeras pas n'importe quoi.

H-10-07 Pisca lis. oun 


\subsection{Les indices de redondance}

Les indices de redondance découlent de la reprise par le locuteur de ses propres paroles. Ces indices sont utilisés vers la fin de son récit lorsquill n'a plus rien de nouveau à ajouter. Il paraphrase donc ses propos comme pour signaler à linterlocuteur, de façon plus insistante, que le tour est terminé et que celui-ci peut prendre la parole, puisque le locuteur n'a plus de nouvelles informations à ajouter.

\section{Exemple:}

H-04-04 Mon oncle, une fois, y voulait m'faire essayer sa 303, j'pense, t'sais, une espèce de grande balle, une balle qui mesure à peu près trois pouces de long, là.

H-04-07 Fait que $y$ arrête, pis y m'installe.

...

H-04-09 Y minstalle.

*..

H-04-13 Si jl'avais accroché, là, j'pense qu'il serait rien resté du siffieux.

H-04-14 303, j'pense y chassait l'orignal avec ca.

H-04-16 Y s'ralt pas resté grand-chose.

4.2.2.1.5 Les signaux explicites de finalité ou mots clés

Nous regroupons dans celte catégorie deux éléments importants, soit les questions directes ou indirectes d'une part, et les mots ou les expressions qui expriment directement la finalité d'autre part. Ces signaux sont un peu différents des autres indices de conclusion, car ils veulent dire explicitement: « j'ai fini de parler et tu peux prendre la parole » et ne donnent donc guère le choix à l'interlocuteur. 


\section{Exemple:}

...

F-06-08 Alors à ce moment-là bon tas t'as plus de stabilité, t'as juste mouvement des vagues qui est imprimé par euh les les conditions euh climatiques mais c'est ça finalement c'est euh l'élément intéressant de la voile.(fin)

$\cdots$

\section{Exemple ${ }^{12}$}

$\ddot{*}$

$F$

Ouh là là. L'année passée là ah, y a deux y a deux ans on était allées en Floride Martine, Marie-Jo pis moi. Fait qu'un un moment donné les filles disent : « ah on va sortir, on va sortir » alors on demande euh où est-ce qu'y avait, j'te l'al tu conté?

4

Non.

\subsubsection{RECENSEMENT DES INDICES SÉMANTICO-PRAGMATIQUES}

Nous avons regroupé les résultats du recensement de ces éléments dans un tableau présenté en ANNEXE E. II est important de rappeler que le corpus sur lequel nous avons travaillé est essentiellement constitué de récits d'événements de la vie quotidienne, ce que Labov (1978) appelle les « récits d'expériences personnelles $\%$, et que nous demeurons consciente que les conversations en général ne prennent pas toujours la forme d'un récit.

Nous avons séparé les différents indices selon 5 catégories. Les 4 types dinndices de conclusion (la conclusion, le jugement de valeur, la redondance et les signaux explicites de finalité ou mots clés) ainsi que les indices de non-conclusion y sont bien représentés. Le Tableau 12 vient en quelque sorte résumer la distribution des éléments sémantico-pragmatiques dans les tours qui sont présentés à l'annexe $\mathrm{E}$.

${ }^{12}$ Cette catégorie n'est pas présente dans les énoncés retenus pour l'analyse, mais elle se retrouve dans l'amorce du tour F-10. 


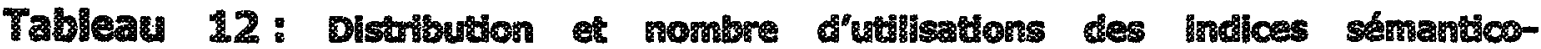
pragmatigues par categorie dans les tours de parale pour les locuterss M et 8

\begin{tabular}{|c|c|c|c|c|c|}
\hline $\begin{array}{l}\text { Tour de } \\
\text { parole }\end{array}$ & Conclusion & $\begin{array}{l}\text { Jugement } \\
\text { de valeur }\end{array}$ & Redondance & $\begin{array}{l}\text { Signaux } \\
\text { et mots } \\
\text { ciés }\end{array}$ & conclusion \\
\hline $\mathrm{H}-01$ & 2 & & & & 1 \\
\hline $\mathrm{H}-\mathrm{O} 2$ & 1 & & 1 & & 1 \\
\hline $\mathrm{H}-03$ & 1 & 1 & & & 4 \\
\hline H-04 & 3 & 3 & 3 & & 5 \\
\hline$* \mathrm{H}-05$ & 1 & 1 & 1 & & 3 \\
\hline H-06 & & 2 & & & 3 \\
\hline$H-07$ & 1 & & 2 & & \\
\hline $1-08$ & 3 & & & 1 & 3 \\
\hline H-09 & & & 1 & & \\
\hline $1-10$ & 1. & 1 & 1 & & 2 \\
\hline$* F-01$ & & & 1 & & 6 \\
\hline F-02 & & & & & 2 \\
\hline$F-03$ & 1 & & 1 & & 1 \\
\hline$* F-04$ & 1 & & 2 & & 3 \\
\hline$F-05$ & 1 & 2 & 2 & 1 & 1 \\
\hline$* F-06$ & & 2 & & 2 & 5 \\
\hline$F-07$ & & & & 1 & 4 \\
\hline F-08 & 1 & & & & 1 \\
\hline$F-09$ & & 2 & & & 1 \\
\hline F-10 & & 1 & & & 5 \\
\hline
\end{tabular}

* Dans ce tour, des indices de condusion sont émis avant certains indices de non-conciusion. Ils font lobjet d'un commentaire particulier à la section 4.2.2.2.1 Les tours typiques et atypiques.

Ce tableau montre d'abord que l'on retrouve la présence d'au moins un indice sémantico-pragmatique de conclusion dans pratiquement tous les tours de parole, seul le tour F-02 fait exception. On observe également que l'intervention de ces indices est dans la majorité des cas cumulative, le locuteur additionnant plusieurs de ces indices, souvent appartenant à des catégories différentes, vers la în de son discours. Il ne faut pas perdre de vue non plus que ces indices de conclusion interviennent après que le locuteur a fait usage, dans les énoncés précédents, d'indices de non-conclusion signifiant explicitement qu'l devait garder le tour de parole. En faisant ainsi usage dindices de conclusion succédant à des indices de non-conclusion, le locuteur s'assure, selon toute vraisemblance, un maximum de 
coopération de la part de son interlocuteur assurant ainsi la fuidité de l'échange. Il s'agit là de la structure idéale, pour ainsi dire typique, de l'échange conversationnel, mais qui connaît néanmoins quelques accrocs dans des tours atypiques.

\subsection{Les tours typiques et atypiques}

Le Tableau 12 en conjonction avec l'annexe E nous ont permis de constater qu'il existe des tours que nous pouvons identifier, faute de terme meilleur, comme typiques et certains autres qui sont en quelque sorte atypiques. Sont considérés comme typiques, sur le plan du fonctionnement sémantico-pragmatique, les tours à lintérleur desquels une succession d'indices de non-conclusion assurent la continuité avant que les indices de conclusion interviennent dans les derniers énoncés. Le Tableau 13 montre un exemple de tour « typique ».

Tableau 13 : Exemple de tour typlque

\begin{tabular}{|c|c|c|c|c|}
\hline $\begin{array}{l}\text { Enonas. } \\
\text { du tour }\end{array}$ & Récit et indices de non-conclusion & Conclusion & $\begin{array}{l}\text { Jugement de } \\
\text { valeur }\end{array}$ & $\begin{array}{l}\text { Interventions } \\
\text { de F }\end{array}$ \\
\hline H-03-01 & Ma fin d'semaine à c.. & & & \\
\hline H-03-02 & $\begin{array}{l}\text { Sais-tu que c'matin id, jtais dans la } \\
\text { douche, pls f'me suls dit : bon, la } \\
\text { C'matin a va mposer des questions. }\end{array}$ & & & \\
\hline H-03-03 & Gu'est-ce gu'a va me demander? & & & \\
\hline $1-03-04$ & $\begin{array}{l}\text { Ava m'demander si jsuis passé proche } \\
\text { d'la mort, a va midemander si... }\end{array}$ & & & \\
\hline$F$ & & & & Non! (rires) \\
\hline$H-03-04$ & Ial dit : attention. (rires) & & & \\
\hline H-03-05 & & $\begin{array}{l}\text { Jilavais préparée } \\
\text { celle-là. }\end{array}$ & & \\
\hline H-03-06 & & & Cest dommage. & \\
\hline
\end{tabular}

Dans le cas des tours atypiques, on observe au contraire un certain enchevêtrement des deux types dindices, des indices de non-conclusion peuvent prendre place après les premiers indices de conclusion et éventuellement alterner 
jusqu'à la conclusion finale. C'est ce que l'on peut observer dans le tour H-05 en exemple dans le Tableau 14.

Tableau 14: Exemple de rour atyplque

\begin{tabular}{|c|c|c|c|c|c|}
\hline $\begin{array}{l}\text { Emonce } \\
\text { du tour }\end{array}$ & $\begin{array}{l}\text { Recte et indices de mon- } \\
\text { conclusion }\end{array}$ & Conclusion & $\begin{array}{l}\text { Jugement } \\
\text { de valear }\end{array}$ & Rediendance & $\begin{array}{l}\text { Interventians } \\
\text { de F }\end{array}$ \\
\hline H-05-01 & $\begin{array}{l}\text { Mais, jvoulais ramener aे } \\
\text { Claude euh, un comet que mon } \\
\text { once fat. }\end{array}$ & & & & \\
\hline$H-05-02$ & $\begin{array}{l}\text { Y prend, y se prend de liécorce } \\
\text { de, de bouleau là, pls y se fait } \\
\text { un beau comet à peu près gros } \\
\text { d'même, pis lę, y remplit ça } \\
\text { d'beurre d'érable. }\end{array}$ & & & & \\
\hline$F$ & & & & & $O n !(b c)$ \\
\hline $4-05-03$ & & & $\begin{array}{l}\text { Ca fait } \\
\text { écoeurant. } \\
\text { (rirest) }\end{array}$ & & \\
\hline H-05-04 & $\begin{array}{l}\text { Ben de sucre, ouais, de sucre } \\
\text { dur pluttot. }\end{array}$ & & & & \\
\hline$F$ & & & & & Ouais ? (bc) \\
\hline $\mathrm{H}-05-05$ & & & & Ca fait super bon. & \\
\hline H-05-06 & $\begin{array}{l}\text { Cest dans un comet de } \\
\text { bouleau pis (hési.) pour faire } \\
\text { tenir le, le comet, là, (resp.) ... }\end{array}$ & $\begin{array}{l}\text {...y met une } \\
\text { p'ttte éclisse de } \\
\text { bois, là, une } \\
\text { p'ttite écharde } \\
\text { dans l'bois. }\end{array}$ & & & \\
\hline
\end{tabular}

Sl on regarde bien le Tableau 13 et le Tableau 14, on voit que les tours qui y sont présentés sont constitués différemment. Dans le premier, les indices de conclusion apparaissent après que tous les indices de non-conclusion nécessaires au suivi du récit du locuteur ont été envoyés. C'est pourquoi nous avons nommé ce genre de tour un tour typique, puisque dans un monde idéal, tous les tours de parole seraient constitués de cette manière. Dans le Tableau 14 , on voit un tour atypique qui ne présente pas une répartition aussi nette entre les indices de non-conclusion et les indices de conclusion. Dans ce tour, le locuteur fait une alternance entre les deux types d'indices: récit+non-conclusion, non-conclusion, jugement de valeur, non-conclusion, redondance, non-conclusion, conclusion. Dans la réalité, plusieurs tours de parole peuvent être constitués de cette façon et il y a au moins deux raisons à cette situation. 
Les deux facteurs que nous avons associés à cette alternance entre les indices de non-conclusion et de conclusion sont liés aux participants à la conversation et entraînent le même résultat. Le premier facteur est à chercher du côté du locuteur lui-même. En cours de conversation, celui-ci après avoir émis un ou plusieurs Indices de conclusion peut soudainement amener la conversation sur un autre sujet ou bien il veut peut-être tout simplement ajouter de nouvelles informations à son récit. C'est ce qui se produit par exemple avec le tour $\mathrm{H}-05$. On se retrouve alors avec une fausse fin dans le tour de parole. Pour ce qui est du deuxième facteur, II semble lié au fait que linterlocuteur ne veut pas prendre la parole même si le locuteur lui a fait clairement savoir quîl voulait la lui céder (tours F-01, F-04, H-08). Face à ce refus de prendre la parole, le locuteur poursuivra alors son tour, sur le même sujet en émettant encore plus de signaux de conclusion ou bien il reprendra sur un tout autre sujet. Il en résulte donc une fausse fin et un tour « artificiellement prolongé ».

Le tour $\mathrm{H}-08$ est un bon exemple de fausse fin provoquée par linterlocuteur. Le contenu indique clairement que le locuteur, très tôt dans le tour, souhaitait céder la parole, mais que l'interlocuteur n'était pas prêt à la prendre à ce moment. 


\section{Exemple: 1 -08}

F Comment ca?

Hos-01 Non, non, j'aurais... jaurais pu.

H-08-02 Ben, mon père enselgnait là ça m'aurait fait un lift (rires) mais autrement on s'était arrangés autrement.

H-08-03 Ma soeur allaik déjà à Garneau, ma mère travaillait à Saint-Sacrement.

F O.K.

H-08-04 Fait qu'on... J'ai dit : j'vais aller à Garneau, ca va être plus simple.

f-08-05 Pis on faisait la run, j'faisais la run de lait, là, le matin.

F O.K.

H-08-06 Déposer ma mère, après ça ma soeur pis le soir je reprenais ma soeur, ma mère souvent.

F Ah, oul. (BC)

H-08-07 ...en auto ...mais en fait, c'est ca, c'est que finalement on avalt une auto pour les trois qui se promenaient dans frcoin de Garneau -- Saint-Sacrement. (FAuSSE FIN)

H-08-08 Pis mon père avait l'autre auto.

F

Ah. (BC)

H-08-09 Fait que c'est ca.

H-08-10 Mais si j'étais allé à Limollou euhy, j'connais quasiment tous les profs de français.

H-08-11 J'aurais eu euh, j'aurais eu du fun dans leurs cours.

F Hmm, hmm. mais c'est difficile, hein, parce que dans un autre, dans un autre sens si th réussis moins bien euh, t'as plus de pression, hein.

On voit clairement, à l'énoncé H-08-07 que le locuteur était prêt à finir son tour à cet endroit. $F$, ne voulant pas prendre la parole ici, n'émet qu'un signal de backchannel forçant ainsi $\mathrm{H}$ à poursuivre son tour. Il reprend alors avec des informations nouvelles à l'énoncé $\mathrm{H}-08-08$ qui est la déduction logique de ce qu'il disait précédemment. Cet énoncé est considéré comme un indice de conclusion puisquill vient confirmer une information déjà sous-entendue par les propos qu'il tenait, comme pour insister sur le fait qu'il a fini. À cet endroit, $F$ n'émet encore qu'un simple signal de back-channel. H se voit alors encore une fols obligé de poursuivre son tour. Il ajoute un autre indice de conclusion encore plus fort à $\mathrm{H}$ 08-09, il utilise « Fait que c'est ça. » appuyé d'une intonation descendante. N'ayant 
toujours pas de réponse, il poursuit enfin sur un autre sujet, comme pour se relancer dans autre chose. Ce n'est qu'après l'énoncé H-08-11 se terminant sur une intensité très faible que $F$ prend finalement la parole. C'est dans une situation de ce genre que les rôles tenus par les participants à la conversation semi-dirigée semblent peut-être interférer. SI on ne sait pas exactement pourquol $F$ ne voulait pas prendre la parole dans ce tour, on présume que son rôle en tant qu'intervieweuse peut influencer dans une situation de ce genre et qu'elle voulait ainsi laisser parler le locuteur le plus possible sans intervenir.

Qu'ils soient typiques ou atypiques, les tours présentent généralement des indices de non-conclusion comme de conclusion. Le locuteur utilise ces signaux pour faire comprendre ses intentions à son interlocuteur. En revenant au Tableau 12, on peut se rendre compte que tous les tours de parole à l'étude, sauf 2 (F-02 et F-07), comptent au moins un des 4 indices de conclusion identifiés, c'est-à-dire soit le dévoilement de la conclusion, l'expression d'un jugement de valeur, la présence de redondance ou l'émission d'un signal explicite de finalité. Il arrive même assez souvent que les locuteurs utilisent plusieurs indices de conclusion différents dans un même tour. C'est le cas des tours $\mathrm{H}-03$ et $\mathrm{H}-05$ déjà donnés en exemples dans le Tableau 12 et le Tableau 13, mais aussi des tours H-04, H-10 F-05 et F-06. Regardons de plus près dans l'exemple qui suit ce qui se passe dans le tour F-05. 


\section{Exemple:}

H Ben oul. Y'a travaillé là-bas beaucoup Charpentier, ça c'est supposé être un spécialiste de cette région-là.

8-05-00 La Nouvelle-Calédonie?

H Hm hmm.

8-05-01 Ah ouais! y parait que c'est un un, c'est vraiment merveilieux hein.

H parait. (bc)

F-05-02 Comme euh comme endroit, ça fait que...

F-05-03 Ben c'est ça été un peu euh et pis bon (NC) euh j'pense que leur décision après ca a été a été finale euh dans le sens quil sont jamais y ont jamais décidé d'y retourner ou entrepris même de des démarches pour y retourner.

F-05-04 Mais c'tait vraiment dommage euh...

F-05-05 Pas que j'aurais aimé ca naître dans un pays exotique, mais c'est que j'aurais aimé ça connaiture autre chose.

H Hm hmm. (bc)

F-05-06 T'sais ça aurait été un choc assez important là pour mes parents par exemple partir de Cap Chat en Gaspésie pis se ramasser en Nouvelle-Calédonie.

F-05-07 R'marque que c'était à peu près dans des conditions de brousse là mais euh mon père allalt enseigner à ce moment là. (redondance avec le tour précédent)

F-05-08 J'pense c'aurait été blen aussi pour euh... (uggement de valeur)

F-05-09 C'aurait peut-être été bien pour mes parents (redondance)

finalement. (mot de)

H

Çaurait été combien de temps? Deux ans?

Le tour se déroule de façon assez régulière sans que la locutrice ne donne beaucoup dindices de non-conclusion (un seul à F-05-03). À partir de l'énoncé F-05-07 on peut remarquer que les indices de conclusion apparaissent et se succèdent. Le premier indice est une redondance avec le tour précédant (F-04), une information qui était déjà connue de l'interlocuteur. Ensuite, il y a un jugement de valeur sur la pertinence de voyage. À F-05-09, la locutrice répète le jugement de valeur qu'elle vient de faire, on a alors un indice de redondance, et, dans la même phrase, il y a la présence du mot clé « finalement ». À la suite de tous ces signaux de finalité, le tour de parole change de locuteur sans problème. 
Le tour $F-02$, se falt au contraire remarquer par l'absence d'indice de condusion.

\section{Exemple:}

H Qu'est-ce qu'y a à voir là? Y a pas grand chose!

7-02-01 Ben, les...la nature, (h : les glaces) les glaces euh, si t'as la chance j'sais pas moi, si d'aller dans un... t'sais dans un... j'aimerais beaucoup voir le le la période de de printemps là-bas.

F-02-02 J'pense que ce qui a fait euh 'tout cas s... ça m'a toujours intéressée, mais j'ai vu un film qui s'appelle « un homme parmi les loups qui est Inistoire d'un scientifique qui va et pis moi bon ça m'a fascinée complètement.

F-02-03 Les paysages qu'on voyait ctait vraiment c'que j'avais envie de voir.

F-02-04 J'aimerais beaucoup faire de la photo aussi.

H Ah oui? (BC)

F-02-05 Ahhh oul.

F-02-06 T'sais pis les euh... en tout cas tout ce qui tout ce qui peut se photographier là, j'aimerais ça.

F-02-07 Puis euh aussi euh... ce que j'aimerais visiter... (fin)

H Mais les pays de l'Est y paralt que, j'connais des gens qui sont allés pis y parait que ben tout se fait en tour guidé là évidemment.

Ce tour est caractérisé par une énumération de ce que la locutrice aimerait faire. Avant que son interlocuteur ne lui coupe la parole, la locutrice hésite déjà beaucoup dans ses propos ayant pour conséquence que le tour prend fin avant même que la locutrice ne soit prête à le céder. C'est probablement pour ceite raison qu'on ne relève aucun indice de conclusion, les hésitations ${ }^{13}$ pouvant provoquer des changements de tour accidentels si elles s'accumulent vers la fin du tour.

C'est un phénomène que l'on observe au tour F-07 où la locutrice cède volontairement son tour de parole avant d'avoir fini son récit. Ce tour ne comporte en fait qu'un seul indice de conclusion, mais très explicite, qui est une demande

\footnotetext{
${ }^{13}$ Voir la partie 3.3.2.2 Les hésitations.
} 
indirecte d'information. La locutrice qui parle depuis très longtemps (le tour dure 66 secondes) cherche une information importante et sollicite en quelque sorte l'aide de linteriocuteur, lui cédant ainsi la parole.

En somme, tout comme c'était le cas pour les indices prosodiques, il ne semble pas que l'on puisse parler d'une utilisation systématique et catégorique d'indices sémantico-pragmatiques pour signaler de façon certaine et non ambiguë la fin d'un tour de parole. On peut cependant observer qu'une majorité de tours semble correspondre à ce qu'on peut appeler un modèle typique. Généralement, ces tours se caractérisent par la présence en cours de route d'un certain nombre d'indices de non-conclusion assurant la continuité avant que n'apparaissent de façon plus ou moins récurrente différents indices de conclusion; en fait, tous les tours de notre corpus présentent au moins un de ces indices de conclusion, à l'exception de F-02 qul se termine abruptement avant que le locuteur n'ait eu le temps de signaler quil était disposé à céder la parole. En opposition avec les tours terminés accidentellement, Il a les tours qui présentent de fausses fins. Nous avons vu que ces fausses fins ne tiennent pas tant à une mauvaise utilisation des signaux de conclusion ou de non-conclusion qu'à un refus de linterlocuteur de prendre la parole ou encore à l'ajout dinformations nouvelles par le locuteur qui se ravise et décide quîl ne cède plus la parole après avoir émis les signaux de conclusion qui ont laissé croire quill était disposé à le faire. Cela nous amène à conclure que les indications sémantico-pragmatiques jouent bel et bien un rôle de structuration des échanges en annonçant la fin des tours de parole, ce rôle, tout comme celui des indices prosodiques ou syntaxiques, ne peut être considéré comme contraignant pas plus que suffisant. Nous pouvons tout au plus considérer que ce jeu d'indices prosodiques, syntaxiques ou sémantico-pragmatiques crée les conditions favorables à un changement de tour, car la réalisation effective de ce changement de tour reste cependant imprévisible et sous le contrôle des protagonistes. 


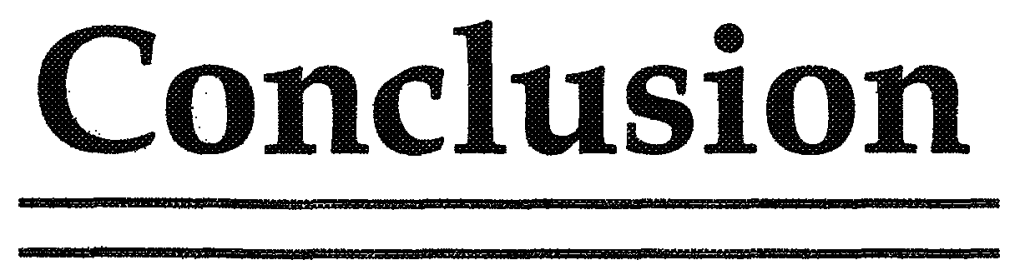


Notre étude sur les éléments influençant la fin des tours de parole avait comme but premier didentifier les éléments prosodiques utilisés par le locuteur pour signaler la fin d'un tour de parole. Pour ce faire, nous avons choisi de vérifier la présence en fin de tour de certaines caractéristiques prosodiques pouvant être considérées comme des indices que les locuteurs utilisent, consciemment ou non, pour signaler cette fin. Le corpus retenu est constitué de deux entrevues semidirigées où la relative familiarité des locuteurs nous place devant un discours très spontané sans aucun contrôle sur le contenu de la part de l'expérimentatrice, contrairement aux principales études antérieures qui impliquaient pour la plupart de fortes contraintes soit sur le type de discours et sur le contenu (Lehiste, 1975) soit principalement sur le contenu (les travaux de Swerts, 1993, 1997, 1998; Swerts et Collier, 1992; Swerts, Collier et Terken, 1994; Swerts, Bouwhuis, Collier, 1994 ainsi que Swerts et Geluykens, 1994) ${ }^{14}$.

L'originalité de notre recherche se situe sur deux plans. D'une part, une étude prosodique portant sur un discours spontané non contraint n'est pas monnaie courante; en raison de son caractère imprévisible, ce type de discours implique forcément une plus grande diversité d'indices prosodiques et non prosodiques qu'un discours contrôlé. D'autre part, la langue étudiée, le français, constitue en elle-même une particularité, les études étant faites jusqu'à maintenant surtout sur l'anglais et le néerlandais, deux langues dont la structure prosodique est assez différente de celle du français.

Au départ, nous avons fait l'hypothèse que les locuteurs annonçaient la fin de leur tour et nous avons cherché à identifier les moyens linguistiques qu'ils utilisaient. Pour ce faire, une série dinndices prosodiques, syntaxiques et sémanticopragmatiques ont alors été considérés. Par la suite, nous avons cherché à savoir si

\footnotetext{
${ }^{14}$ Swerts et son équipe ont principalement travaillé sur des discours expérimentaux dont le contenu est plus ou moins prédéterminé, du type instructions d'assemblage, description de figures géométriques, itinéraires et description de peintures.
} 
certains de ces indices étaient plus udulisés que les autres et dans quelle mesure Pobjectif était de voir s'il y avait un élément qui serait plus important.

La dimension prosodique constitue le premier et le principal champ d'investigation de notre recherche. Dans cette perspective, nous avons d'abord repéré et mesuré la longueur des pauses pour ensuite étudier leur rôle dans le fonctionnement des tours de parole. Après analyse, les résultats obtenus apparaissent s'écarter sensiblement de lidée généralement admise qui voudrait que les fins de tour soient accompagnées d'une pause plus longue. En effet, les pauses de fin de tour de notre corpus sont en général plus courtes que les pauses entre énoncés contrairement à ce qu'avance Rémi-Giraud (1987). Nos observations vont cependant dans le même sens que les résultats de Swerts et Geluykens (1994) et de Swerts (1997). Nous avons fait I'hypothèse que cette différence de comportement des pauses pourrait découler du fait que les fins de tour sont préalablement annoncées d'une façon ou d'une autre ; dans ces conditions, les pauses peuvent être moins longues à cause de l'anticipation provoquée par les différents éléments linguistiques utilisés par le locuteur pour signaler que le tour arrive à sa fin et qưll souhalte céder la parole à un interlocuteur. De plus, notre corpus, même sill reste spontané, autant au plan du discours que du contenu, présente toutes les caractéristiques d'un échange coopératif où les participants n'ont pas vraiment à « défendre » leur tour de parole, comme ce pourrait être le cas dans un débat ou un échange argumentatif ; une pause, même relativement longue, à Iintérieur du tour n'impliquerait pas, dans ces conditions, un risque important de coupure de la parole.

L'analyse de lintonation a pour sa part été réalisée sur trois niveaux : global, intermédiaire et local. Au niveau global, l'étude a révélé qu'll y aurait une tendance générale à l'abaissement de la fo tout au long d'un tour de parole dans $65 \%$ des

${ }^{15}$ Les participants sont moins en compétition pour le tour que s'ils étaient partie prenante d'un débat. 
cas, ce qui rejoint les observations de Lehiste (1975), Lieberman, Katz, Jongman, Zimmerman, Miller (1985) ainsi que de Swerts, Collier et Terken (1994). Dans la même veine, on a pu constater que les voyelles finales de tour présentent le plus souvent une FO plus basse que les voyelles finales des énoncés intratour. Sur les plans intermédiaire et local, les résultats obtenus sont par contre beaucoup moins nets, les énoncés finals et intratour se comportant relativement de façon similaire.

If est cependant important de souligner qu'au plan local, contrairement à nos attentes, les schémas présentant une orientation montante sont les plus souvent utilisés aussi bien pour les énoncés finals (53\%) que pour les énoncés intratour (59\%). Parmi ces intonations montantes, un schéma intonatif s'est démarqué des autres. En effet, on a pu observer une nette prédominance pour le schéma descendant-montant ( $\backslash$ ) fortement représenté pour les deux types d'énoncés avec des résultats respectifs de $40 \%$ pour les énoncés finals et de $36,8 \%$ pour les énoncés intratour. Cette prédominance globale des schémas ascendants est encore plus surprenante quand on considère que seulement $20 \%$ des énoncés finals se terminent avec un schéma intonatif descendant, un taux même inférieur à ce qu'on observe pour les énoncés intratour qui, eux, présentent un taux de $22 \%$ de schémas intonatifs descendants.

L'élément intensité, de son côté, nous montre des résultats qui, de prime abord, semblent assez positifs et concluants. La majorité des énoncés finals de notre corpus $(70 \%)$ présente en effet une valeur en dB plus basse que celle de la moyenne des énoncés du tour. On retrouve également $55 \%$ des tours caractérisés par une descente globale de la courbe d'énergie, ce qui va dans le même sens que Laver (1994). Pour ce qui est de l'intensité de la voyelle finale de tour, elle est plus basse que la moyenne d'intensité des voyelles cibles de son tour dans une proportion de $85 \%$. Il convient cependant de limiter la portée de cette dernière affirmation, à la lumière du fait que seulement $30 \%$ des voyelles finales de tour présentent une intensité inférieure à lintensité moyenne des voyelles finales 
d'énoncé intratour, ce qui suggère que cette baisse dintensité finale serait plutôt une proprété de fin d'énoncé peu importe sa position dans le tour (Ouellon, 1990).

En somme, les indices prosodiques retenus pour notre étude montrent que les énoncés finals auraient, jusqu'à un certain point, des caractéristiques qui peuvent les distinguer des autres énoncés. Mais aucun de ces éléments prosodiques ne semble constituer un indice catégorique de fin de tour, c'est-à-dire un indice qui serait régulièrement présent et qui signalerait de façon non équivoque la fin du tour. En effet, non seulement leur présence n'est pas obligatoire, mais ils sont aussi susceptibles d'apparaitre ailleurs à lintérieur du tour, sans que leur occurrence ne soit démarcative, sans compter que l'on peut souvent douter du caractère perceptible de la variation portée par cet indice, compte tenu de son ampleur et de la dimension de l'unité sur laquelle elle s'observe ${ }^{16}$.

D'autres indices, notamment les indices syntaxiques et sémantico-pragmatiques, ont été pris en considération de façon complémentaire. Du côté des indices syntaxiques, les énoncés finals et les énoncés intratour présentent encore une fois des caractéristiques assez similaires. On peut cependant retenir que les énoncés finals étalent plus souvent incomplets que les énoncés intratour dans 5 cas sur 20 contre 3 sur 131 pour les énoncés intratour. Cette différence est proportionnellement assez importante (25\% pour les énoncés finals contre $2 \%$ pour les autres), si on considère que les énoncés intratour sont beaucoup plus nombreux. Elle est aussi surprenante si on se rappelle que la complétion fait partie des 6 indices « cède-tour » identifiés de Duncan (1972: 287). Il faut cependant prendre en considération le fait que, contrairement à ce qui se passe chez Duncan, la plupart des énoncés incomplets ne sont pas le résultat d'une interruption forcée où linterlocuteur « vole» le tour de parole, mais interviennent plutôt dans un

\footnotetext{
${ }^{16}$ Une diminution de $10 \mathrm{~dB}$, par exemple, va sembler beaucoup plus forte sur un tour de 2 ou 3 énoncés que sil cette variation se produit sur un tour de 10 énoncés. L'étendue de l'unité sur laquelle la variation s'applique peut donc jouer un rôle important dans la perception qu'on aura de cette variation.
} 
contexte de plétinement de la part du locuteur, plus ou moins arrivé au terme de son propos, ce qui emmène linterlocuteur à intervenir pour lui venir en aide en le déchargeant de la parole ou en lui donnant linformation recherchée.

Les indices sémantico-pragmatiques sont eux aussi bien présents dans les tours de parole de notre corpus, mais il semblerait que leur fonctionnement ne soit pas plus explicite ou catégorique, sans toutefois être aléatoire ni imprévisible. À la lumière de nos observations, ces indices auraient une fonction plus incitative que contraignante sauf peut-être pour certains signaux explicites de finalité comme une demande d'aide de la part du locuteur à l'interlocuteur.

Somme toute, l'étude d'un corpus naturel laisse voir une interaction complexe de plusieurs éléments autant prosodiques que syntaxiques et sémanticopragmatiques. Les résultats que nous avons obtenus nous amènent à nuancer les résultats d'études expérimentales telles que celles de Swerts et ses collaborateurs qui suggèrent un rôle direct et important des indices prosodiques pour signaler la fin du tour. Il faut cependant considérer que de tels résultats ont été obtenus dans un contexte où les indices prosodiques étaient les seuls à l'étude, les autres indices ayant été neutralisés par un contenu déterminé à l'avance. De plus, le design expérimental de plusieurs de ces études invitait explicitement les sujets à utiliser des stratégies prosodiques connues pour marquer les frontières d'énoncé ou de topiques, ce qui ne peut qu'accentuer l'effet des facteurs prosodiques. Nos résultats nous portent à croire que, dans la « vraie vie », c'est-à-dire dans l'usage naturel du langage, on aurait plutôt affaire à la mise en place d'un environnement favorable au changement de tour plutôt qu'à un seul type d'indices bien définis. Le locuteur émettrait une série d'indices prosodiques et linguistiques que linterlocuteur devrait repérer pour comprendre qu'il a la possibilité de prendre la parole, mais en demeurant toutefois libre de saisir ou non l'opportunité qul lui est offerte. Cela n'est pas sans rappeler les conclusions de Duncan (1972) qui identifie six indices « cède-tour» se conjuguant ensemble pour permettre au locuteur 
d'annoncer la fin d'un tour de parole. Linterlocuteur aurait ainsi plus de facilité à déceler la fin d'un tour si le nombre dindices est élevé. Il y aurait là une piste à explorer avec un corpus plus étendu et surtout en cherchant à établir de façon expérimentale, au moyen de tests de perception, le poids des divers facteurs et de leur combinaison sur la capacité des sujets à percevoir et à identifier correctement la fin des tours de parole. 


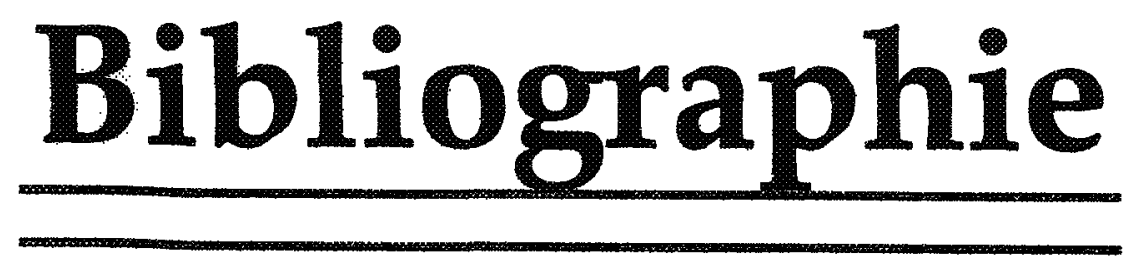


BESTGEN, Y. (1998). Segmentation markers as trace and signal of discourse structure, Journal of Pragmatics, vol. $29, n^{\circ} 6, p .753-763$.

TEN BOSCH, L., N. OOSTDUJ et L. BOVES (2005). On temporal aspects of turn taking in conversational dialogues, Speech Communication, vol. 47, p. 80-86.

BRUCE, G. et P. TOUATI (1992). On the analysis of prosody in spontaneous speech with exemplification from Swedish and French, Speech Communication, vol. 11, p. 453-458.

COUIER, R. et J. 'T HART (1975). The role of intonation in speech perception, dans A. COHEN et S.G. NOOTEBOOM (dir.), Structure and process in speech perception, New York, Springer-Verlag, p. 107-123.

CRYSTAL, D. (1985). A dictionary of linguistics and phonetics, $2^{e}$ édition, New York, Basil Blackwell Ltd, 340 p.

CUTLR, A. et M. PEARSON (1986). On the analysis of prosodic turn-taking cues, dans C. JoHN-LEWIS (dir.), Intonation in discourse, London, Croom Heim, p. 139-155.

DEMERS, M. (1996). (Socio)prosodie, syntaxe et discours, Le cas du discours rapporté en français québécois oral spontané, Thèse de doctorat inédite, Université Laval, Québec, 333 p.

Di CRIsTo, A. (1985). De la microprosodie d̀ lîntonosyntaxe, tome 1, Aix-enProvence, Publications de l'Université de Provence, $854 \mathrm{p}$.

DOLBEC, J. et C. OuElLoN (1987). Structures de la phrase française, Chicoutimi, Gaëtan Morin Éditeur, 293 p.

DUCHESNE, L. (1991). Les marqueurs acoustiques de lénoncé en francais québécois, Mémoire de maîtrise inédit, Université Laval, Québec, 141 p.

DUNCAN, S. JR. (1972). Some signals and rules for taking speaking turns in conversations, Journal of Personality and Social Psychology, vol. 23, $n^{0} 2$, p. 283-292. 
FónAGY, I. et 3. FónAGY (1983). Lintonation et l'organisation du discours, Bulletin de la société de linguistique de Paris, tome 78, fasc. 1 , Paris, Klincksieck, p. 161-209.

Fontaney, L. (1987). L'intonation et la régulation de l'interaction, dans J. COSNIER et C. KERBRAT-ORECCHIONI (dir.), Décrire la conversation, Lyon, Les Presses Universitaires de Lyon, p. 225-267.

FrIES, C.C. (1952). The structure of English, an introduction to the construction of English sentences, New York, Harcourt Brace \& World Inc., 304 p.

GELUYKENS, R. et M. SWERTS (1993). Local and global prosodic cues to discourse organization in dialogues, ESCA Workshop on Prosody 1993, Working Papers, vol. 41, Lund, Dept of Linguistics and Phonetics, Lund University, p. 108-111.

GELUYKENS, R. et M. SWERTS (1994). Prosodic cues to discourse boundaries in experimental dialogues, Speech Communication, vol. 15, p. 69-77.

HIRSCHBERG, J., D. LITMAN et M. SWERTS (2004). Prosodic and other cues to speech recognition failures, Speech Communication, vol. 43, p. 155175.

Krahmer, E., M. SWeRTS, M. Theune et M. Weegels (2002). The dual of denial: Two uses of disconfirmations in dialogue and their prosodic correlates, Speech Communication, vol. 36, p. 133-145.

LABOV, W. (1976). Sociolinguistique, Paris, Les Éditions de Minult, 458 p.

LABOV, W. (1978). Le parler ordinaire, la langue dans les ghettos noirs des États-Unis, vol. 1, Paris, Les Éditions de Minuit, 351 p.

LAFOREST, M. (1992). Le back-channel en situation d'entrevue, Québec, Centre international de recherche en aménagement linguistique, coll. Recherches sociolinguistiques, Université Laval, 231 p.

LANDERCY, A. et R. RENARD (1977). Éléments de phonétique, Bruxelles, Didier, $214 \mathrm{p}$. 
LAVER, J. (1994). Principles of phonetics, Cambridge, Cambridge University Press, $685 \mathrm{p}$.

LEHISTE, I. (1975). The structure of paragraphs, dans A. COHEN et S.G. NoOTEBOOM (dir.), Structure and process in speech perception, New York, Springer-Verlag, p. 195-206.

LEHISTE, I. et W. S.-Y. WANG (1977). Perception of sentence boundaries with and without semantic information, dans DRESSLER, W. V. et O. E. PFEIfF (dir.), Phonologica 1976, Innsbruck, p. 277-283.

LEHISTE, I. (1979). Perception of sentence and paragraph boundaries, dans LINDBLOM, B. et S. ÖHMAN (dir.), Frontiers of speech communication research, Londres, Academic Press, 393 p.

LeHISTE, I. et G. E. Peterson (1972). Some basic considerations in the analysis of intonation, dans D. BOLINGER (dir.), Intonation, Baltimore, Penguin Books, p. 367-384.

LEPAGE, M-J. (1996). Étude des facteurs prosodiques influençant la perception des fins de tour de parole, Dialangue, Bulletin de linguistique, Université du Québec à Chicoutimi, vol. 7, p. 118-121.

LEPAGE, M.-3. (2000). Étude des éléments prosodiques influençant la perception des fins de tour de parole, dans É. KavanaGH (dir.), Actes des XIIF Journées de linguistique, Québec, Centre international de recherche en aménagement Iinguistique, Université Laval, p. 101-110.

Lieberman, P., W. Katz, A. Jongman, R. Zimmerman et M. Miluer (1985). Measure of the sentence intonation of read and spontaneous speech in American English, Joumal of Acoustical Society of America, vol. $77, n^{\circ} 2$, p. 649657.

Matasci-Galazz, E. et E. Pedoya-Guimbretière, (1987). À l'écoute de Bernard Pivot: une stratégle de hiérarchisation des informations par la prosodie, Études de linguistique appliquée, $n^{\circ} 66$, p. 106-117. 
Molbaek Hansen, P., N. Reinholt Petersen et E. Spang-Hanssen (1993). Syntax, pauses, and temporal relations in the final part of the sentence, ESCA Workshop on Prosody 1993, Working Papers, vol. 41, Lund, Dept of Linguistics and Phonetics, Lund University, p. 160-163.

OuEuloN, C. (1990). Les marqueurs acoustiques de lénoncé en discours québécois spontané, Actes des XVIIF Journées d'études sur la parole, Montréal, 28-31 mai 1990, Montréal, Université de Montréal et Société française d'acoustique, p. 26-30.

OueloN, C. (1991). L'énoncé en discours oral spontané, Revue québécoise de linguistique théorique et appliquée, vol. $10, n^{\circ} 3$, p. 61-84.

OUELION, C., C. PARADIS, et L. Duaresne (1991). Les marqueurs acoustiques de l'énoncé en français québécois, Actes du XIF Congrès intemational des sciences phonétiques, Aix-en-Provence, Publications de IUniversité de Provence, vol. 2, p. 430-433.

PIERREHUMBERT, J. (1979). The perception of fundamental frequency decination, Joumal of the Acoustical Society of America, vol. 66, $n^{\circ} 2$, p. 363-369.

RÉMI-GIRAUD, S. (1987). Délimitation et hiérarchisation des échanges dans le dialogue, dans ]. COSNiER et C. KeRBRAT-ORECCHIOni (dir.), Décrire la conversation, Lyon, Les Presses Universitaires de Lyon, p. 17-72.

Rossi, M. (1999). Lintonation, le système du francais: description et modélisation, Paris, Éditions Ophrys, 237 p.

SACKS, H., E. A. SCHEGLOF et G. JeFfirSON (1974). A simplest systematics for the organization of tum-taking for conversation, Language, Joumal of the Linguistic Society of America, vol. L, $n^{\circ} 4$, p. 696-735.

SWERTS, M. (1993). On the prosodic prediction of discourse finality, ESCA Workshop on Prosody 1993, Working Papers, vol. 41, Lund, Dept of Linguistics and Phonetics, Lund University, p. 96-99.

SWERTS, M. (1997). Prosodic features at discourse boundaries of different strength, Joumal of the Acoustical Society of America, vol. 101, p. 514 521. 
SWERTS, M. (1998). Filled pauses as markers of discourse structure, Joumal of Pragmatics, vol. 30, p. 485-496.

SWERTS, M., D.G. BOUWHUIS et R. COUIER (1994). Melodic cues to the perceived "finality" of utterances, Joumal of the Acoustical Society of America, vol. 96, p. 2064-2075.

SWERTS, M. et R. COLUER (1992). On the controlled elicitation of spontaneous speech, Speech Communication, vol. 11, p. 463-468.

SWERTS, M., R. COLUIER et J. TERKEN (1994). Prosodic predictors of discourse finality in spontaneous monologues, speech Communication, vol. 15, p. $79-90$.

SWERTS, M. et R. GELUYKENS (1994). Prosody as a marker of information flow in spoken discourse, Language and Speech, vol. 37, $n^{\circ} 1$, p. 21-43.

SWERTS, M. et M. OSTENDORF (1997). Prosodic and lexical indications of discourse structure in human-machine interactions, Speech Communication, vol. 22, p. 25-41.

SWERTS, M., A. WicHMANn et R.-J. BEUn (1996). Filled pauses as markers of discourse structure, Proceedings of the Intemational Conference on Spoken Language Processing, October 1996, Philadelphie, vol. 2, p. 1033-1036.

TAYLOR, T.J. et D. CAMERON (1987). Analysing conversation. Rules and units in the structure of talk, New York, Pergamon Press, 178 p.

TouaTr, P. (1991). Analyse de la prosodie de la parole spontanée en suédois et en francais, Actes du XIF Congrès international des sciences phonétiques, Aix-en-Provence, Publications de l'Université de Provence, vol. 4, p. 282-285.

TOuAT, P. (1993). Prosodic aspects of political thetoric, ESCA Workshop on Prosody 1993, Working Papers, vol. 41, Lund, Dept of linguistics and phonetics, Lund University, p. 168-171. 
Toukn, P. (1995). Pitch range and register in French political speech, Proceedings of the International Congress of Phonetic Sciences 1995, Stockholm, vol. 4, p. $244-247$.

YNGVE. V.H. (1970). On Getting a Word in Edgewise, Papers from the Sixth Regional Meeting Chicago Linguistic Society, April 16-18 1970, Chicago, Chicago Linguistic Society, p. 567-578. 


\section{Annexe A}

Corpus $H$ 


\section{Légende:}

\begin{tabular}{|c|c|}
\hline 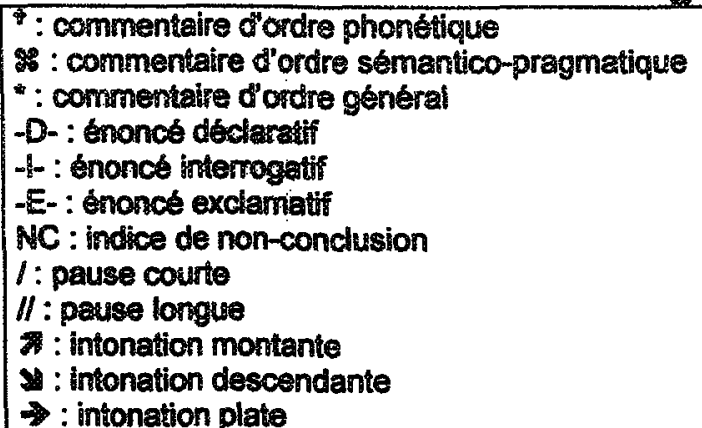 & $\begin{array}{l}\text { Voyelles cibles: } \\
\text { PrEmière de tour ou après une pause : majuscule + } \\
\text { gras + souligné } \\
\text { Anté-pénutieme et pénultieme avant UnE pause et } \\
\text { voyelle avant une pause interne : majuscule + gras } \\
\text { Derniere de tour : majuscule + gras + souligné } \\
1,2 \text { ou } 3 \times \text { même voyelle : nombre de fois où la valeur } \\
\text { de la voyelle a été utilisée dans le calcul par manque } \\
\text { de variéte. } \\
+1,+2,+3 \text { avant : nombre de syllabes que nous avons } \\
\text { reculé pour trouver une valeur que CSL pouvait lire. }\end{array}$ \\
\hline
\end{tabular}

\section{LOCUTEUR 1}

H-01

Durée : 19,54 sec.

H Ouais. Chance j'ai pris un cafe. Ouais.

Tenregistes, lá ?

F Hmm, hmm.

H O.K. Ce qui est arrivé avec la gomme /

[DÉBUT DU CORPUS]

H-01-01 c'est qu'tu m'As faite penser quand 34 syllabes

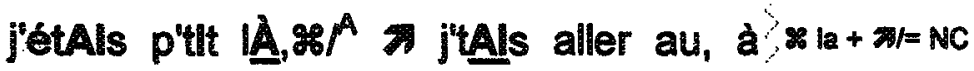

l'hôpital avec ma mère pour me faire

brâler des verrues sur l'colN dEs

E望vres.// $/^{B}-D^{16}$.

H-01-02 T'sAls, y brolaient ça avec euh, de 29 syllabes

lazote liquide là, j'pense, pi elle avait:

dit: si tu pleurs pas, j'vais t'dOnnER

une gomme. If $y-D$.

Enonce de la promesse. Conclu

H-01-03 Pls effectivement, comme de faite, 49 syllabes

c'était à l'hôtel Dieu, j'pense, à fin là,

\footnotetext{
${ }^{16}$ Tout au long du corpus il y a des lettres qui indiquent le type de phrase : D=déclarative, I=interrogative, $E=e x c l a m a t i v e$
} 
Gans Ivieux Québec là, à la fin là, quand mes verrues avaient été brulées, Dévoilement de la conclusion.

javais pas braille, j'avais eu mEs dEUX, "il n'y a pas de voyelle sur le signal.

p'tites* gQtmmes Chicklets*.ll

(BACK-CHANNEL) -D. 4 syllabes

+2 avant, $2 x$ merme voyelle.

H-01-04 JHAls bEN content. $/ / \%$ (FIM) -D.

$*$ conclusion

F Hey, tu devais être heureux là.

H Oui. C'était super.

H-O2

Durée: $19,25 \mathrm{sec}$

$\boldsymbol{F}$

Ouan, mais sauf qu'eux autres y ont comme un, un, un régime reconnu pour ça, t'sais.

H-02-01 Oui, oul, je l'sais ben, mais t'sais, c'est,

23 syllabes

c'est pas mieux euh, c'pas pire parce ça arrive aux EtAts Unis.//

$F$

Hmm. (BC)

H-02-02 La, c'pArce y en ont bAttU UN, $f^{A} 37$ syllabes mAls en Afrique du Sud, quand $y$ arrêtent, quand y a des émeutes pis o dovoliement do la conclusion qu'y arrêtent dEs gENs, $\mid \mathbf{A} / /^{\beta} \not$, Eh! caline, $y$ font battre vraimENit. $F$, Mais puisque cells-ci met trop de temps a calline, y se font battre Vraimeni, il reprend la parole. II fait la

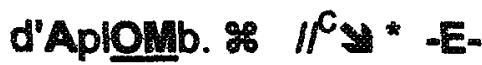
description de l'assaut.

H-02-03 Y'ONt de grands bâtons, là, pis y 17 syllabes

frappent, là. Sont cinq ou six à frAppER il semble que pendant cetie phrase $F$ veullile d'ssús.l \& (FIM) -D.* prendre la parole. Peut-etre parce quilis sont dans une situation plus argumentative. 
F Mais aux États Unis c'est, t'sais ta, tes supposé davoir un, un régime démocratique.

$\underline{H-03}$

$\mathbf{F}$

Faif que c'est ça. Hey, raconte-moi ta, ta c, ta in d'semaine à cabane à sucre.

\section{H-03-01 MA fin d'sEmAine A c.." $\rightarrow$}

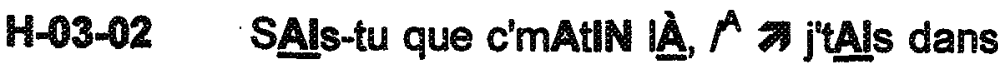
la douche, pis j'mE suls dilt : $\beta$ mbON, $F$

* IA c'matin a va m'poser dEs: quEstioNs. ${ }^{D}$ y-DC.

H-03-03 Qu'Est-ce qu'a va m'dEmANdER?* -1D- 6 syllabes * Question thétorique H-03-04 A va m'demander si j'suis passé proche; 25 syllabes d'la mort, a va m'demander si ${ }^{A_{*}}$ (rires) "lai F répond en quelque sorte aux - interrogations de $\mathrm{H}$. Cette intervention de $\mathrm{F}$ $\left(F \rightarrow\right.$ Nonl) Là jai dit : AttENtiON ${ }^{B} / f^{*}$ n'a pes été comptée comme un changement (rires) -DC.
H-03-05
J'I'Avais préparÉe cElle-IÂ./ H Y -D-
Ah oui?
H-03-06
7 syllabes.
Superposition avec F.
H indice de conclusion
$\mathbf{F}$

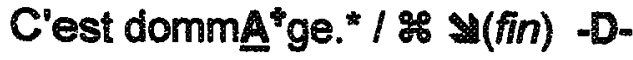
3 syllabes.
superposition avec F.
+ 3x méme voyelle
2o jugement de valeur

5 syllabes

* Faut debut. Le locuteur reflechit à ce que F lui demande. II vout soudainement raconter ,ce qu'll avait on tate le matin de l'entrevue. II y a alors un changement de topique qui s'en suit.

25 syllabes de tour de parole puisque $H$ n'en tient pas compre. Le a non \$ de F survient pendant que $\mathrm{H}$ rit et celui-ci poursuit son discours sans probleme.

*B Ici on note une pause meme s'il n'y a pas vraiment de silence. La pause est remplie par les rires de $\mathrm{H}$. 
$H \quad$ Oui. (rires)

F Ah, ben jhe la demanderai après.

H-04

$F$

H-04-01 FAlt que ce.. "

H-04-02 BEN*, j'voulais, j'cherchais une cible pis 22 syllabes je, mais j'aime pas ça tirer sur les p'tites bêtes, pAr ExEMple. / 1 -D-

$\mathbf{F}$

H-04-03 J'ai"me mieux tirER dans un plt dE 9 syllabes sAble.// ע (resp.) -D-

H-04-04 MON oncle, une fois, y voulait m'faire 36 syllabes essayer sa trois cent trois, j'pense, t'sais, une espèce de grande balle, une balle qui mesure à peu près trois pOUces dE IONg, lä. L $/ / \hbar$ (resp.) - "Prononed très faiblement. D.

H-04-05 PIs on cherchAlt quElque chose $. \rightarrow-6$ syllabes D. meme valeur de fréquence mais 3 voyelles différentes.

H-04-06 FAlt que on passe à cótÉ d'UN chAMp 20, $I^{A} \rightarrow$ pls y dit: Tiens, y dit, y a des siffleux dANs ce chAMp $\mid \AA^{B}$-DC. $\quad *^{B} N C$

H-04-07 FAlt que y Arrête, $A^{A}$ \& $\pi$ pls y 8 syllabes 


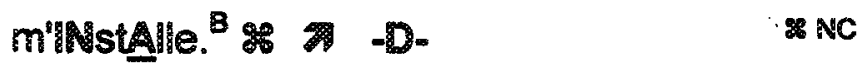

H-04-08 Pis y avAlt justementa un siffeux qui

etait debout sur ses pattes dEN NC

Arrięre.// -D.

H-04-09 Im'binstalle.

H-04-10 j'A passé, la j'ai tiré pis, je, je, j'a 3 syllabes

rasé le siffleux j'pense, j'ai passé juste 26 syllabes

rase le siffleux, j'pense, j'ai passé juste $x$ conciusion

AU-dessus d'IA tÊte. \$

H-04-11 En tous CAs, y avait un trou dans terre,

là, long de même, j'pense, la balle étAlt 19 syllabes

rENtrÉe. \& /72-D.

H-04-12 Le siffEUx ESt part tout de sugte.l/

-D.

8 syllabes

ante-penultieme +2 avant

H-04-13 Si jlil'Avais accroche, là, j'pense qu'il;

serait rien rEstÉ du siffEUX*. Hugement de valeur

IT)(rires)

* meme valeur de fréquence mais voyelles différentes

H-04-14* Trois cENt trois, j'pense y chassait:

lorignAl AvEc ça. \% //ע -D- (- fort) 13 syllabes

H-04-15 Euh j'tIrAls s'UN siffEUx. \& / (rires) -

D- $\quad$ redondance + jugement de valeur

H-04-16 Y s'rAlt pas reste graNd chose. $1 /$ (fin) $-\mathrm{D}$

7 syllabes

* jugement de valeur

- A l'oreille, linto. semble descendre +bas dans le dernier segment que dans les autres.

$F$

Le, le, l'objectif était disproportionné par rapport

* F ne semble pas certaine de la în du tour. Elle semble attendre avant de prendre is au calibre, hein." parole. Apres un cumul d'indices de finalité divers, elle finit par prendre la parole. C'est peut-etre do à son róle d'intervieweure dans la conversation.

\section{H-O5}

Durée: $21.67 \mathrm{sec}$.

$H$

$Y$ étaient pas pire, hein ?*

* Le tour commencerait a cet endrot, mais puisque je crois que le * Ohl n de F est émis pour répondre a la question de $\mathrm{H}$ il ne seraft 
$F$

MAls, j'voulais ramener à Claude euh,
un comet que mON ONcle fAlt. $-\mathrm{D}$ -
H-05-02 $\mathrm{Y}$ prENd, y se prend de l'écorce de, de bOUIEAU, $\mid A, f^{A}$ \& $\approx$ pls y se fait un beau cornet à peu prÊs gros d'mÊme, $P^{B}$ : 7 pis A, y remplit ca d'bEUrre d'ÉrAble.// -D-

$F$

H-05-03 CA fait ÉcoEUrANt. / (rires) \% -D.

H-05-04 BEN de sucre, ouais, de sucre dUr plutôt.// =-D. *

$\mathbf{F}$

H-05-05

$H-05-06$
Oh!* (BC)
Ouais ? (BC)

$$
\text { CA fait sUpEr bON./ * * } \rightarrow \text {-D-(-fort) }
$$

alors pas un back-channel mais plutd: un tour de parole, il remplace le oul.

$\therefore 5$ syllabes

33 syllabes

$212+2 /=$ NC

mame valeur de fréquence mais voyelies differentes

- lai, Hine prend pas yraiment en compte le - Oh! que F émet. C'est un back-channel et Pautre pas. II sert en quelque sorte à inciter la locuteur a poursuivre et a lui indiquer qu'elle le suit toujours.

\section{5 syllabes}

* jugement de valeur

9 syllabes

*Fausse fin?

5 syllabes

Xredondance+ jugement de valeur -F essaie de dire quelque chose.

34 syllabes

bOUIEAU pis $A$ (hési.) pour faire

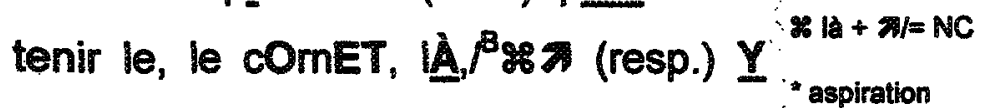
met une p'tite éclisse de bois, là, une p'tite échArde dANs l'bOls. $\mathcal{C}$ y $(F / N)^{*}$ - Farole. Elle ne laisse pas une longue pause Dapres son intervention ef reprend a nouveau la parole. Elle ne laisse pas à $H$ le temps de reprendre la parole. 
H-06

Durée: $24,31 \mathrm{sec}$.

F Tu y vas tu a toutes les annees ?

H-06-01 NON, ${ }^{A}$ ac'est IA premiÈre AnnEÉe. ${ }^{B} \Rightarrow 7$ syllabes

-D.

H-06-02 SAls-tu que ça fait cinq ans au moins 30 syllabes

quE j'mE dils : IA \& a j'vAls m'prendre NC

une semaine, pls j'vAls allER/ $/^{\beta}$

AldER mON ONcle/I T A faire ses, $P$ Mome si $\$$, la phrase est incomplete a cot

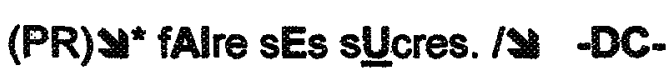
endroit et la pause est remplie, co qui laisse entendre que $\mathrm{H}$ hesite.

H-06-03 Puis c'ESt la premiere année que j'peux 20 syllabes

$y$ aller euh, trois jours pendant l'tEMps

dEs sücres. I\$ $-D$ -

F Hmm, hmm. (BC)

H-06-04 *Pis Autrement, là, $A$ H $\rightarrow(P R)$ euh 15 syllabes

Te délai entre H-06-03 et -04 est trés court

j'peux jamais y aller À chAque Année. 1 \& a tout juste le temps d'émettre un BC.

H-06-05 MAls, t'sais, ça arrive toujours dans une

24 syllabes

période où c'est en fin de session, les

travaux sONt $A$ r'mEttre. $/ / \rightarrow(B C) \quad-D$ -

H-06-06 Là, c't'Année j'ai pas eu trop une grosse session, A fIN, IA. // \& $\pi$-D-

14 syllabes

8 la $+\pi j=$ NC

5 syllabes

X jugement de valeur

H-06-07 Fait que j'n'Al profitÉ. / \& y -D-

H-06-08 ÇA première fOls dEpuis IONgtemps ${ }^{*} / 8$ syllabes

I (FIM) -D- (-fort)

Cette phrase est prononcere pius faiblement que les autres.

$F$

Tes parents étaient déjà là ? Y étaient déja 
descendus?

H-07

Duree: $18.61 \mathrm{sec}$

$F$

Ah, ben, c'est pas pire ça.

H-07-01 OuAl's. / $\-D$ -

1 syllabe

$\$ 3 x$ mente voyelle

H-07-02 Fait qu'c'Est çA.* -D-

3 syllabes

H-07-03 Pis en fin d'semaine, c'est ça mon Conclusion du sujet précédent.

oncle, c'était l'seul qui avait pas détaillé 21 syllabes

j'pENse, du colN. //7 -D-

H-07-04 TOUs les autres avaient dÉtAllEE.I ॠ 8 syllabes

D.

8yllabes

F Ah, oui ? (BC?)

H-07-05 PArce qu'y a fait euh, ouit y a eu des, y

a eu des grosses journées de chaleur 27 syllabes là-bas aussi, là, quinze à viNgt dEgrÉs. F. H sent le besoin de donner des

// 7 -D.

F Hmm, hmm. (BC)

H-07-06 Tout I'mONde pensais qu'les sucres étAlent finis. \&

10 syllabes

H-07-07 Tout ImoNde a dÉtAillÉ. / 1 (resp.) $x$

D. $\quad x$ redondance

H-07-08 MON oncle a attENdu UN PEU, ${ }^{A} / 7$ pis 28 syllabes

A. y a ramassé euh, y va avoir fait

Honctusion

plusieurs galons en fiN de sEmAlne.

H -D- (fin)

F La manne. Ah, ben c'est lfun. 
H-OS

Durée: $34,42 \mathrm{sec}$.

F Commentça?

H-08-01 NON, non, jaurais.. jAUrAls pU./ -D- 7 syllabes

H-08-02 BEN, mon père enseignait là $(B C)^{A}$

ca m'aurait fAlt UN $\| \mathrm{ft}^{\mathrm{B}} / \mathrm{T}$ (RIRES)

mais* Autrement on s'était arrangés Rires pardessus mais.

AutrEmENt. ${ }^{c}-\mathrm{D}$ -

H-08-03 MA soeur allait déjà à Garneau, ma 19 syliabes

mère travaillait à Saint-SAcrEmENt. I\$

D.

F O.K. (BC)

H-08-04 FAlt qu'on. j'ai dit: j'vais aller a 15 syllabes

Gameau, ça vA Être plus simple*. $/ /{ }^{*+2}+2$ avant

D-

"Ce n'est qu'un bruit sur le signal, il n'y a pas du tout de voyelle qui apparaite

H-08-05 Pis ON faisait la run, j'faisais la run de 16 syllabes

IAlt, IA, \& le mA*tW. I/ $-D$

\% NC

* ll y a un bruit par dessus le a, c'est pour cette raison que nous avons pris la donnee de la voyelle de lait.

$F$

O.K. $(B C)$

H-08-06 Déposer ma mère, après ça ma 22 syllabes

soeur 7 pis $\%$ le soir je reprenAIS $\mathrm{mA}$

SOEUr, ${ }^{A} / \mathrm{TA}$ mÈre souvENt. $\boldsymbol{\beta}^{\mathrm{B}} \mathrm{N}-\mathrm{D}$ -

F $\quad$ Ah, oui*. (BC)

- Ce n'est pas une question.

H-08-07

...en auto ...Mais EN fait, c'est ça, $\boldsymbol{y}^{* t}$

34 syllabes

c'est que finalement ${ }^{\prime}$ on avait une

AUto pour $/ \rightarrow$ IES trois qui se moldé 


\begin{tabular}{|c|c|c|}
\hline & $\begin{array}{l}\text { AUtO pOU } \$ \text { IES trois qui se } \\
\text { promenaient dans l'coin de Ganeau- } \\
\text { Saint-SAcrEmENt. } 1 \text {-D. }\end{array}$ & \\
\hline K-08-88 & $\begin{array}{l}\text { Pis moN père avAT IAUtre au } 10^{*} .1 / \\
\text { Ah. }(B C)\end{array}$ & 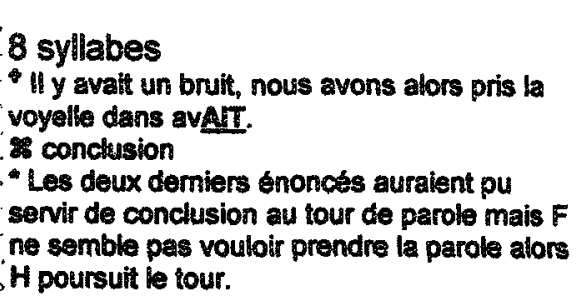 \\
\hline H-08-10 & $\begin{array}{l}\text { Fait que* c'Est çA./\$ \& } \text {-D. } \\
\text { MAls si j'étais allé à Limoilou euh, } \\
\text { j'connais quasiment tous les profs. dE } \\
\text { rANçais*. } \rightarrow-D \text { - }\end{array}$ & $\begin{array}{l}3 \text { syllabes } \\
\text { Ily avait une superposition avec } F \text { ici, nous } \\
\text { n'avons pas pu relever les données de ces } \\
\text { voyelles. } \\
\text { conclusion (il sert d'amorce pour ce qui va } \\
\text { suivre.) } \\
21 \text { syllabes } \\
+ \text { fort (comme un \& reset } \text { ) } \\
\text { Bruit de micro trop fort. }\end{array}$ \\
\hline H-08-11 & $\begin{array}{l}\text { JAUrAIS EU euh, }{ }^{A} / / \rightarrow \text { j'AUrais eu du } \\
\text { fun dANs IEUrs COUrs. } \$ / / Y \text { \& }-\mathrm{D} \text { - }\end{array}$ & $\begin{array}{l}12 \text { syliabes } \\
\text { Prononce très falblement. } \\
\text { conclusion }\end{array}$ \\
\hline$F$ & $\begin{array}{l}\text { "Hmm, hmm. Mais c'est difficile, hein, parce } \\
\text { que dans un autre, dans un autre sens si tu } \\
\text { reussis moins bien euh, t'as plus de pression, } \\
\text { hain }\end{array}$ & $\begin{array}{l}\text { "Finalement } F \text { se décide à prendre la parole } \\
\text { ce que semblait vouloir } H \text { depuis déja } \\
\text { quelques énoncés. }\end{array}$ \\
\hline
\end{tabular}

H-09

Durée: 13,99 sec.

F Comment ça?

H-09-01 BEN, j'avais, j'avais d'la pression, $\pi$;2 syllabes

Patrlck AUss! I I -D-

H-09-02 ON pouvait pas euh, on pouvait pas s'planter d'IN trAvAUx. // 7 -D-

H-09-03 COnnaissANt CONrAd*. // $/$-D-

14 syllabes

5 syllabes

. La pause est assez longue et fr essaie de prendre la paroie ici mais $H$ reprend le tour aussitot. 
H-09-04 T'sais, quANd tu connais I'prof, tu peux 21 syliabes pas euh, tu peux pas vraiment * F dis quelques chose mais on ne lentend tplantER. T'as d'IA prEssion pas. -1 -

+2 avant

* jugement de valeur

H-09-05 Tu sAls que lui aussi, quand y va 21 syllabes corriger y va sûrement avoir UN p'tt' pEU dE.. $/ \rightarrow(F I N)-D$ -

5 "C'est dur ça, d'écrire en fonction du lecteur, " F coupe la parole è $\mathrm{H}$. hein.

$\underline{H-10}$ Duree : $23.14 \mathrm{sec}$.

$F$

Tas un blocage psychologique?

H-10-01 OuAls. J'pENse quE oul. I/\$ -D. 4 syllabes H-10-02 Est raide Denise, $\approx$ EST AU, ${ }^{A} \approx$ du 19 syllabes côté méthode, tu sais qu'y a rien qui vA. PAsSER. ${ }^{B} / 7-D$ -

$F$

Effectivement. (BC)

H-10-03 T'sAls que si tu fais, si tu fais quelque 21 syllabes chose qui est pas cOrrEct, $\| A, A / \& \nexists c ̧$ $3 \mathrm{~B}+3 /=\mathrm{NC}$ passera pAs pANtoUte ${ }^{B} . / / \triangle D$ -

H-10-04 Par contre,\% tu sais que si tu fais 26 syllabes quelque chose qui est pas correct, pis $8 \mathrm{NC}$ tu linscris, tu lindlques ${ }^{A}, I \rightarrow$ ç va Etre cOrrEct. $^{B} / \mathbf{Y}-D$ -

H-10-05 T'sAls, si tu remarques tes erreurs, si tu reconnais tes ErrEurs $\mid \mathbb{A}, \mathrm{A} / \mathrm{c}$ A va: Être cOrrEct. ${ }^{B}$ H -D. 


\section{Etre cOrrEct.}

H-10-06 SAUf que tu y passera pas n'MPportE 11 syllabes

quoi:. I\$ 20 -

La fin de cette phrase est beaucoup moins forte.

+1 avant

tonciusion

$F$

Ah, non, non. Non. (BC)

$H-10-07$

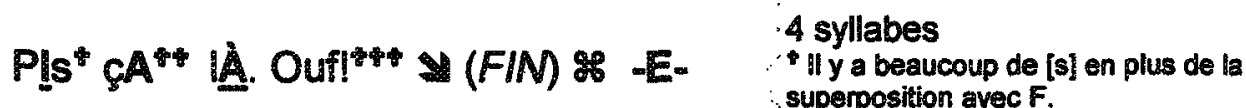

2x meme voyelle

Le oufl de la fin est tres aspire, il n'est

$\mathbf{F}$

Ça c'est un faite, hein. Denise, là. Mais même presque pas sonorisé. Prononce trés faiblement.

euh, meme Conrad aussi. Conrad y est d'une so jugement de valeur logique implacable. 
Corpus F 


\section{LOCUTEUR 2}

Lesende:

${ }^{+}$: commenteire d'ordre phonétique

2 : commentaire d’ordre sémantico-pragmatique

* : commentaire d’ordre général

-D- : énoncé déclaratif

-1- : énonce interrogatif

-E- : énonce exclamatif

NC : indice de non-conclusion

1: pause courte

II: pause longue

$x$ : intonation montante

y : intonation descendante

$\rightarrow$ : intonation plate
Voyelles cibles:
PrËmiere de tour ou après une pause : majuscule + gras + souligné
Antépenutième et penutitieme avant UnE pause et voyelle avant une pause interne : majuscule + gras
Demière de tour : majuscule + gras + souligne
1,2 ou $3 \times$ même voyelle: nombre de fois où la valeur de la voyelle a été utilisée dans le calcul par manque de variété.
$+1,+2,+3$ avant: nombre de syllabes que nous avons recule pour trouver une valeur que CSL pouvait tire. euh...

F-01-01 BEN / 7 pEUt-Être, ${ }^{B} / \approx$ oul $C / \approx$ c'est 58 syllabes ÇA malheureusement IÀ j'pENse quE ch... $D / \Rightarrow$ peut-Etre moins maintenant $a+(a+m /=N C$ cause de la situAtion $\mathrm{A}^{\mathrm{E}}$ \& / $\approx$ mAls. j'AimerAIS CAA $/ \neq$ visitER les pays du bloc de I... ben en tout cas l'ancien blOc dE PEst, ${ }^{G}$ /I $\Rightarrow$ pis euh \& IA Russie, PUnion SoviÉtlquE. ${ }^{H} \| \rightarrow-D$ -

F-01-02 J'AlmerAls ÇA, / ÉtrANgemENT, ${ }^{\mathrm{B}} / 18$ syllabes

* Aller euh dans les régions là euh ÉlOIgnÉES. '// צ -D-

F-01-03 CElles qui sONt AU nOPrd... 5 syllabes

H La Sibérie.*

F-01-04 ...la SibÉrle oul. I T -D.

"Il n'y a pas de changement de tour ici parce que lintervieweur suggere une réponse au locuteur. La locutrice ne code pas la parole, il n'y a meme pas de pause.

5 syllabes 
7-01-05 Pis ON a comme une espece de mythe 15 syllabes autour de IA SibErle. I $\$$-D-

F-01-06 Pis j'Aimerais ça vOlr c'quE c'EST. $/ 3$ syllabes $-D$ -

F-01-07 J'IrAIS IA. I

F-01-08 J'IrAIS $/ \rightarrow$ EUH en AIAsKA, ${ }^{B} / /$

3 syllabes

$*$ NC

j'Almerais beaucOUp volr çA. ${ }^{C}$ - $D$ -

F-01-09 Ch'u plus AttréE pAr euh ${ }^{A} / /$ euh molNs attirée par les pays tropicaux euh la jungle euh pis tout cA molNs. ${ }^{B}$ / D.

F $-01-10$

F-01-19

Thend TANdls quE euh ${ }^{A}$ if $/ \Rightarrow$ plUs par IES 18 syllabes pAYs euh ${ }^{B} \mid$ bON tempérés puis auss $\| A$ frolds ${ }^{C}$. I -D-

13 syllabes

MAIS I'Alaska c'est quElque chOse quE ${ }^{A} \rightarrow$ j'Aimerais bEAUcOUP volr*. -D-H

"Fausse fin?

Is redondance

8 syllabes

X NC question ritetorique

F-01-12 Pls sais-tu c'que j'aimerAis le plus fAlre? " $\rightarrow-28$

F-01-13 Une croisière EN bAtEAUA / qui pArt euh, qui qui existe actuEllemENT $\mid A^{B},{ }^{B}$ I 7 qul part de... ${ }^{c} / I \rightarrow$ (AVALE) dE de 46 syllabes de de de.... de la Colombie-Britanique euh dE VANcouvEr/ $\rightarrow$ ET qui monte jusqu'en AlAskA. ${ }^{\mathrm{E}}$ / y .D.

F-01-14 LA tu fais les côtes d'Alaska 7 etc, Et 45 syllabes cEAtErA, / (RESP.) pls euh tu fais quoi donc, tu j'pense que bon y doit $y$ avoir des escales un moment donné là. d'hésitations bON bEN tu vOIS euh... ${ }^{B} \rightarrow-D-($ FIN)* 


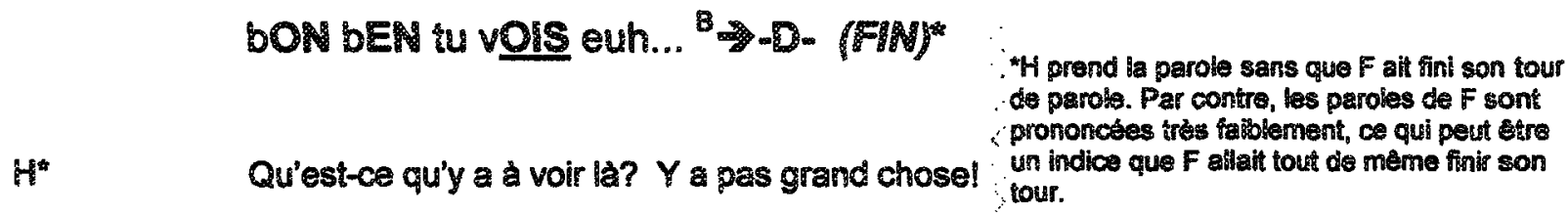

F-02-01 BEN, les...la natUre, (H: les glaces) IEs 40 syllabes glAces euh, ${ }^{A} / \Rightarrow$ si t'As la chance j'sais pas moi, si d'aller dans un... t'sais dans un... j'aimerais beaucoup voir le le la période de de printEMps IÅ-bAs. ${ }^{B}$ / : D.

F-02-02 J'pENse que ce qui a fait euh 'tout cas s... ça m'a toujours intéressée, mais j'ai: 53 syllabes vu un fim qul s'AppElle ${ }^{A}$ «UN. homme parmi les loups» qui est: l'histoire d'un scientiflque qul $v A^{B} \mid \boldsymbol{X}$ (resp.) Et pis mol bON ${ }^{C}$ / çA m'a fascinée complètemENT. ${ }^{0} \rightarrow-D-$

F-02-03 LES paysages qu'on voyait c'tait 15 syllabes vraiment c'que j'avais ENvle de volir. -D-

F-02-04 J'Almerais beaucoup fAlre de IA photo aussi. * /

10 syllabes

- La fin de cette phrase est prononcse plus faiblement.

H

Ah oui? (BC)

F-02-05 Athhh oul. 1 -E-

F-02-06 T'sAls pis les euh... en tout cas tout ce $2 \times$ meme voyelle 
qui tout ce qui peut se photographiER 23 syllabes

IA, ${ }^{A}$ / j'AlmerAS ça. ${ }^{B} /$ -

$2 \pm+2 z=N C$

F-02.07 Puls euh aussi euh... ce quE j'aimerais* visitER... $\rightarrow-D-(F I N)$

12 syllabes

* li y a une hesitation ou on dirait que lo locuteur cherche autres choses à dire.

Prononce tres lentement

Mais les pays de l'Est y paraitt que, j'connais des

gens qui sont allés pis y paraitt que ben tout se fait en tour guidé là évidemment.

Ouais mais la la differrence c'est qu'y ont pu d'économie non plus. Dans l'temps des Tsars au moins...

F-03-01 Hmhum. Effectivement, t'avais, t'avais 23 syllabes un un, t'avais un espèce de fONDs d'AssurÉ IÀ. I Y -D-

F-03-02 BEN y partaient pas de rien hein, t'sAlS 11 syllabes C'EST IES... $1 \rightarrow$-D-

F-03-03 Alors que maintenant ben c'est vraiment y a pu riEN qui EST EN ${ }^{\star A} / \approx$ y $A$ : 40 syllabes strictement rien qui leur pErmET dE...*B $\|$ d'Assurer une économie ou de garantir CErtAlnes choses. ${ }^{C}$-D.

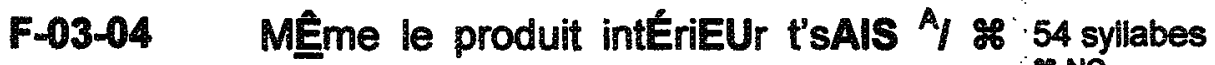
s...quAND \& y arrivent même pas a

produire assez pour nourrir la population, pis a produire assez d'auto, pis a produire t'sais $\%$ tout ce qui EST 
pis a produire t'sais $\mathscr{H}$ tout ce qui EST:

prodult eun ${ }^{3} \mid \Rightarrow$ cOmmerciAlsÁble $y$ besucoup d'hésitations

Y.. ${ }^{* C}-D-(F(N)$

H Pas juste les produits alimentaires.

F-04

H

F-04-01 Ah c'est une longue histoire la Nouvelle- 13 syllabes
CalÉdOnle. -D- $\mathscr{H C}$

F-04-02 C'est pArce quE... ${ }^{A} \|$ quAND... ${ }^{B} \mid 30$ syllabes AvANT que j'nAlsse... $\|$ \& euh mON +1 avant père avait eu un contrat pour aller enseigner en Nouvelle-CalÉdŌnie ${ }^{D_{*} / /}$ -D-

F-04-03 Puls euh finalement ben ils étaient bon y 44 syllabes étaient prếs à partir là t'sais c'tait c'était "anté-pénultième et pénulitieme +2 avant plUs oul IÀ euh ${ }^{A} / \rightarrow$ pis $Y$ préparaient vendre, y s'préparaient à vendre la: mAlsON pis tout çA. ${ }^{B} /$ (RESP.) -D-

F-04-04 Pİs un moment donné ma mère s'est 37 syllabes aperçue qu'elle étAIT ENcEINte ${ }^{A} /$ ET c'était de moi qu'elle était enceinte $x$ conclusion ça fait qu'y ont décidé de PAs y AllER. ${ }^{8}$ $B^{*}$ Fausse fin? $\mathrm{H}$ décide de ne pas prendre la // $\$$-D-

$H$ Ah!
Duré: 72,33 sec. parole. 
F-04-05 Pis euh... j'avais un oncle qui était labas dans l'temos y faisait partie des pÈres ObIA ts, ${ }^{A}$ || puls ${ }^{\text {t }}$ euh c'est 25 syllabes

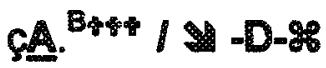
* voyelte penutieme et anté-penulieme qui avance +2 avant.

or Prononce fablement. C'est comme si elle se parlan a alememe.

\& NC

F-04-06 Ç'Avait. y nous $a$, y nous ra, y nous a ramené tellement de choses 68 syllabes merveilleuses là de la NouvelleCalÉdOnle, A dEs, (H: Ah oui?) des photos, les euh, ah toutes sortes de trucs, des coquillAgEs, dES euh, ${ }^{B}$, dEs des des des carapaces dE tortUes euh, ${ }^{C} / \Rightarrow$ Avait tellement de trucs: intÉrEssANTs. $I^{*} \approx-D$ -

- Il y a beaucoup de répetitions et de reprises dans ce tour.

F-04-07 Pls c'tait vraiment charmant parce que $y$ avait UN AccENT I $\approx$ çA faisait, ça 54 syllabes faisait très longtemps qu'y étAT pArtl. ${ }^{B}$ 7 Pis y Avait un accent français mais qui était pas l'accent parisien très fort mais qui était un accent très IÉgER. ${ }^{C}$ //. T-D-

F-04-08 Pis c'tAIT vraiment agréable dE I'ENTENdre. // 7 \%

10 syllabes

se redondance

F-04-09 Y Était prêtre dANS c'tૈEMPS-IÀ.* $*$ || $-D-(F I N)$

6 syllabes

prononce tres faiblement

redondance

$H$

C'est Charpentier qui était qui a travaille beaucoup lá-bas. S.si t’avais été là l'an passé ou y a deux ans taurais pu... 
$8-05$

Duree: $46,49 \mathrm{sec}$

H Ben oui. Y'a travaillé là-bas beaucoup.

Charpentier, ça c'est supposé etre un spécialisto de cette région-là.

F-05-00 LA Nouvelle-CalédOnle? / -8-

7 syllabes

$H$

Hm hmm.

F-05-01 Ah ouAls! ${ }^{A}$ / $Y$ pAraît que c'est un un, 16 syllabes c'est vraiment mervEllEUX helN? ${ }^{B} /$ of

$H \quad Y$ parait. (BC)

F-05-02 COmme euh comme endroit, chA fAIT 8 syllabes quEE... $\rightarrow$ H -D-

F-05-03 BEN c'est ça Été UN pEU euh ${ }^{A} / \rightarrow$ et 61 syllabes pis bON Ho euh j'pense que leur décision après ça a été a été finale euh dans le sens qui sONt jAmAIS ${ }^{B} / y$; ONT jamais décidé d'y retourner ou entrepris mÊme $\mathrm{dE}^{\mathrm{C}} \quad H$ dES démarches pour y rEtOUrnER. ${ }^{D} /$ I $-D$.

F-05-04 MAIS c'tait vraimENT dOmmAge euh... 7 syllabes \& $/ \rightarrow-D$ -

F-05-05 PAs que j'aurais aimé ça naître dans un 25 syllabes pays exotlque mAIS C'EST ${ }^{A}$ que j'Aurais aimé ça connAître Autre chose. ${ }^{B} / /$-D. 
H Hm hmm. (BC)

F-05-06 T'sAlS ça aurait été un choc assez 41 syllabes important là pour mes parents par exemple pArtir $d E^{A} \mid \Rightarrow$ Cap Chat en: Gaspésie pis se ramasser en Nouvelle- $\|$ n'y a pas de pause, $F$ reprend le tour tres CalÉdOnle. ${ }^{*}$-Drapidement

F-05-07 R'mArque que c'était À pEU près ${ }^{A} / 28$ syllabes dANS des conditions de broUsse 1 À mAIS euh ${ }^{B} / \Rightarrow$ mON père allait anté-pénutiòme+2 avant enseigner $\hat{A}^{*}$ ce momENT I $\underline{\Lambda}^{C}$. as /I

D-

F-05-08 J'pENse c'aurait été bien AUssI pOUr 10 syllabes euh... $/ / \rightarrow-D-$ H

se jugement de valeur

F-05-09 C'aurAIT peut-être été bien pour mES 14 syllabes PARENTS ${ }^{*}$ finalement ${ }^{*}$ : $-\mathrm{D}-\%$ redondance (FIN) 2 mot clé

- Prononce trés faiblement et $H$ parle par dessus la fin de ce tour.

H C'aurait été combien de temps? Deux ans?

F-06

Durée: $89,41 \mathrm{sec}$.

H Quel genre de voilier?

F-06-01 AH! mON DIEU. Euh... $\| \rightarrow-E-$

4 syllabes

H Du gros? 30 pieds?*

- Ici le locuteur semble vouloir préciser la question qu'il a posé précedemment.

F-06-02 QuArANte dÉjÀ. // \ -D-

4 syllabes 
H Ah ouais?

F-06-03 MAIS pas AUtANt quE.. ${ }^{A} / \Rightarrow$ IE plus 27 syllabes petit j'pense c'tAIT UN viNGT' $\Rightarrow$ quElques piEds ${ }^{\mathrm{C}} / \rightarrow$ ÇA ça été mes premières, mes premières fOlS $\mathrm{dE}$... ${ }^{D} / \mathrm{c}$ $\Rightarrow-\mathrm{D}$ -

F-06-04 PIs ${ }^{A} /$ j'Avais une frousse, je.j..j..j.j'ai 40 syllabes jamais eu le mal de mer, j'Al jAmAls, ${ }^{B}$ ?: j'étais toujours correcte sAUf quE ${ }^{C}$, T'sAIS j'ai pas d'inconvÉniENT $\mid A^{D} / 2$ aAr rapport euh AUX bAtEAUX. ${ }^{E}$ / -D-

F-06-05 MAls euh c'quE j'AlmAls, ${ }^{A} /$ euh c'quE jaaimais pas par contre c'EST que j'AvAIS $^{B} \mid \rightarrow$ pAs $^{\mathrm{C}}\left|\cdots \mathrm{jE}^{\mathrm{D}}\right|$ 36 syllabes Celte partie de Pénonce est prononcse moins fort et plus rapidement que le reste de la phrase comme pour faire une sorte de commENT j'te dirais", j'comprenais pas IA dynamlque ${ }^{E}$ du bAtEAU sur I'EAU. / Y -D.

F-06-06 Ce qui fAIT que des que le vENT se 76 sy parenthese. IEvAlT, ${ }^{A}$ \& euh j'Avais toujours l'impression que bon on allait verser, on allait couler euh ON AllAIT euh, ${ }^{B} \mid \rightarrow$ t'sAIS quand t'sais un moment donné l'bateau glte pis $|\mathbf{A}| \mathbb{A}^{c} /$ म $\neq m \in$ mé tout ce que t'as rangé là ça s'ouvre pis ça sort, t'en as pArtout IA ${ }^{\mathrm{D}}$ H $\|\approx\| \mathbf{A}$ tu dis t'sais ah que c'est IfUn IA volle. ${ }^{E}$ jugement de valeur 13 -D. \& $121+2+N \mid=N C$

$3(a+7)=N C$

80 sylliabes 
F-06-07 MAßs un moment donné quand j'ai commencé plus a barrer la t'sais a bArreR un p'tit pEU là, / $8 \% 18+71=\mathrm{NC}$ que bon sss ton.le bateau qu'est-ce qui. veut, I'SAIS SON SON ${ }^{3 /}$ sON son point mort c'est de se mettre le nez dans le vent, se dÉvENtER ${ }^{C} /$ puls c'est cette position là qui recherche. Alors que si tu lâches la barre, tu te met le NEZ dANS I'vENT. ${ }^{D}$ / \& $-D$ -

F-06-08 Alors à ce momENT là bon t'as t'as plus 59 syllabes de stabilité, t'as juste finalement $\mathscr{H}$ le mot dé mouvement des vagues qui est IMprimÉ pAr euh / a IES les conditions euh climatlques mAls I $^{B}$ c'EST ça mot clé finalement 28 c'est euh ${ }^{C_{*}} \| \Rightarrow$ Pause très très longue. L'hesshation qui Pí P'ÉlÉmENT / 2 INTÉrEsSANT ${ }^{E}$ / 4 dE que $H$ ne prend pas la parole.

IA volle. F / צ D- 2 (FIN)

\& jugement de valeur

$H$

Mais ça ça doit etre euh, ça se fait beaucoup: des voyages en..en voilier.

H

C'est tout.

F-07-02 Hm. AH, ${ }^{A}$ Is Ah non j'va t'dire avec 56 syllabes Laurence pis un Braque AllemAND IÀ, 


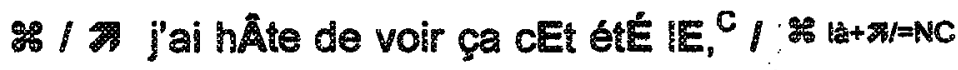

- IE, le cher Laky sur les bakeaux, ben

on a un dériveur au, au lac jpense pas

qu'on lembarque fà-dessus y va: mANger 1 volle. $/-D$

F-07.03 OU y va manger les piÈces du BAtEAU. / -D-

F-07-04 $\mathrm{BOb}$ en a fait des voyages super $\mathrm{EN}$ bAtEAU. I -D-

12 syllabes

H Ah oui?

F-07-05 EUX-autres y ont longtemps eu un, un, UN vOlliER euh ${ }^{A} / \rightarrow$ UN gros voilier qul 24 syllabes AissAlent $t^{B}$ dANS IES BAHAmAs. ${ }^{C}$.

I) $-\mathrm{D}$ -

F-07-06 Puls euh, ${ }^{A} \mid \rightarrow$ puis EN Floride là $y$.76 syliabes faisaient presque toujours ces vOyAges- $|a+\pi|=N C$ $\| A^{B} / H$ puls euh ${ }^{C} / y$ se promenaient; mon Dieu, pis un moment donné, la demière fois qui en a fait un, UN IONG: $\mid \mathrm{A}^{D}$ H / C'EST qu'ils ont ramené le $\mid \mathrm{a}+\mathrm{m} /=\mathrm{NC}$ bateau des BAHAmAs jusqu'A $\mathrm{E} /$ euh...jUsqu'à New York et pis après ça, New YOrk jUsqu'A F/ MONtréAl jE pENse. $/$ / ע $-D$ -

F-07-07 NON, ${ }^{A} /$ H IES Bahamas jusqu'à le 40 syllabes bateau jusqu'A NEW YOrk, ${ }^{B}$ IA HC marina de New Y.., non y ont continué après New York y ont continués, ils l'on. remONtÉ UUSAU'À C TIRFSP .) D. 


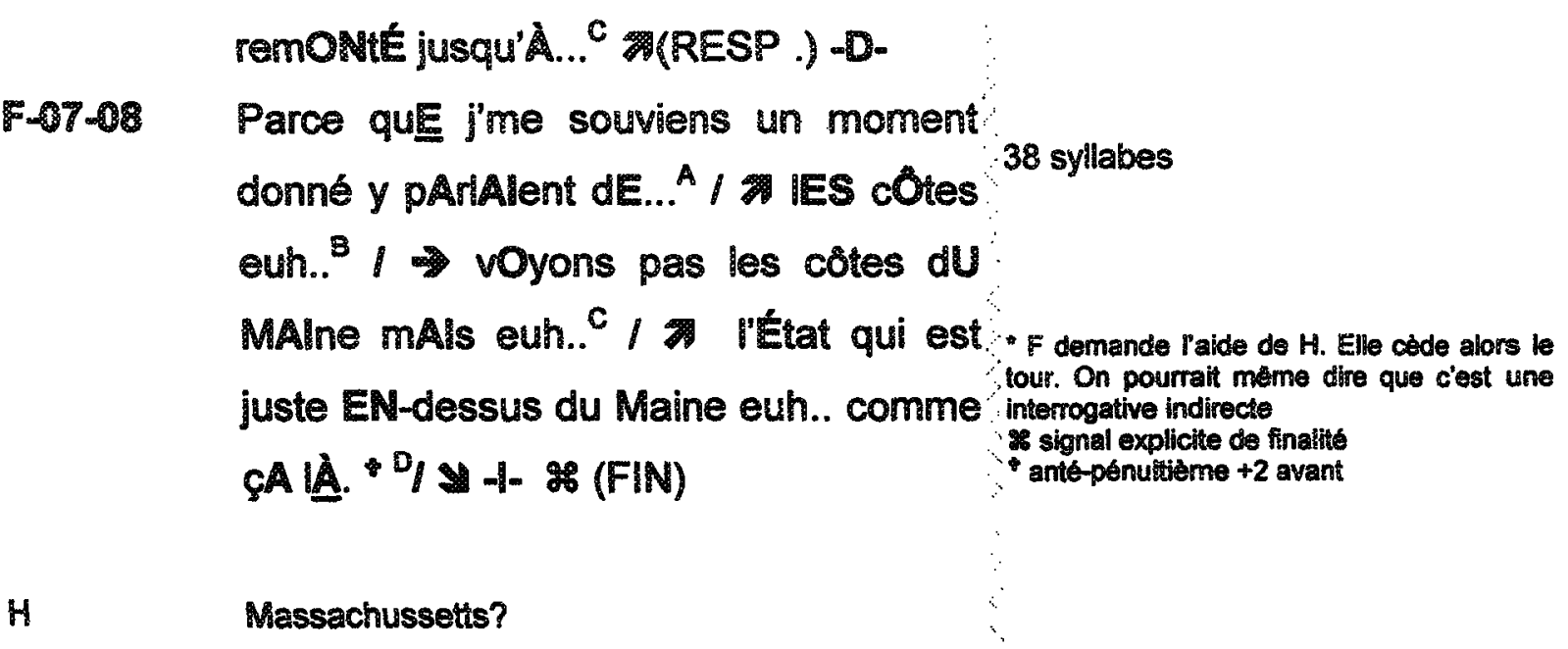

F-08

Durbe: 37.34 sec.

H Cape Cod, ca ca rentre dans la mer comme ça

la

F-08-01 C'EST ça pis y'a une especce tu peux 21 syllabes rentrer à l'intérieur ou couper EN llgne: drolte. / 2 - D.

F-08-02 SI y fait beau tu tu tu pars mettons de la 29 syllabes pointe de Cape Cod pis tu coupes en ligne drotte tu t'EN $v A s^{A} / 2$ tsAls tu fAIS pAs l'ANse. . $^{\text {T-D. }}$

H Ouais.

F-08-03 TANdis que si y fait pas beau tU fAls 10 syllabes I'ANse. / -D-

F-08-04 ET pis eux-autres bon y'était suppose 41 syllabes de de faire beau et pis ils ont ils ont $p A s$ 


\section{F-08-05 FAIT quE."}

TAIT l'AnseA / SAUf que ça a été effrayant y'ont été obligés de rivireR de bord. ${ }^{B} / 4-D$

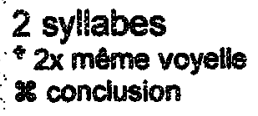

F-08-06 MAlS ga c'est des des des voyages de DAtEAU IA euh.. ${ }^{A} / H \rightarrow$ t'sAIS moi le le marin solitaire là euh attaché après son $M A t \mid A$ pour $P A s^{B} / \rightarrow$ quAND y fait trop mauvais ça me tente pAs vrAlmENT $^{c} /$ mAIS $^{D} /$ IE IE euh.. ${ }^{\text {/ }}$; $\Rightarrow$ bON je sacrifie pas une part de sécurité $\mid A$ pour euh... F/" $\rightarrow$-D- (FIN) etre terrible hein?

F-09*

H Si elle a pas des preuves irréfutables, ça Durée: 26,78 sec.

- F parle plus fort et est plus émotive dans ce tour qui est plus argumentatif que les marchera, ca passera pas.

F-09-01 TOUt A* fAIT. I

F-09-02 Pis d'UN AUtre cÔtÉ, ${ }^{A} /$ a Y sont prêts 3 syllabes $2 \times$ même voyelle à donner la llste $\mathrm{dE}$ tOUtes $/$ / 7 IES 30 syllabes personnes qui sont membres du sénat: qui ont fait un chÈque pAs d'fONDS. ${ }^{c} /$ -D.

F-09-03 J'veux dilre, y ont tous été ÉtUdiANTS. I $\$-D$. 7 syllabes 
5.09-04 LA quelque part un moment donné dANS tA vie, ${ }^{A}$ I jeUX \& dire tu tu 46 syllabes

ca t'arrive de te tromper de compte, ou quoi que CE SOIT, I'SAIS ${ }^{B}$ SONT, ${ }^{C}$

$\Rightarrow$ y ONT une espece $d E d E d E^{D} \| /$ cÔté justicier là qui est mAl pIAcÉ. ${ }^{E}$ / -D-

$H \quad H m .(B C)$

F-09-05 PIs euh j'trouve ça pEsANT, t'sAIS. IH -D. NC

F-09-06 J'pENse que tout compte fait on est bien miEUX Icl A/ qu'AUX ÉtAts- 16 syllabes Unis. ${ }^{B} /$ y \& $-\mathrm{D}-$ (FIN)

H

Ah ça c'est garanti!

Ouh la là. L'année passée la ah, y a deux y a deux ans on étaient allées en Fioride Martine, Marie-Jo pis moi. Fait qu'un un moment donne les filles disent: " wah on va sortir, on va sortir alors on demande euh où est-ce qu'y avait, j'te l'ai tu conte?"

$$
\begin{aligned}
& 7 \text { syllabes } \\
& \text { of jugement de valeur }
\end{aligned}
$$


F-10-01 NON? +1-

1 syllabe

- 3x meme voyelle

H

Vas-y.

F-10-02 ON demande où est-ce qu'y avait une 12 syilabes discothÈque qui ÉtAIT * le fun. I y -D- * +1 avant

F-10-03 ÇA fAIT quE, \& A/ $\pi$ mol ça me tentait 26 syllabes pAs j'Al dit: $:^{3} / 3$ «j'aimerAls mieux $\%$ NC rester euh à l'hótel j'me sens UN pEU fAttiguée.n/ $\mathrm{C} / \mathrm{D}-\mathrm{D}$ -

F-10-04 Y ONT dit: I\% «Non, non, non, c'est 18 syllabes notre avant-dernier soir on va allER

dANSER.» / -D.

F-10-05 CA fait qu'on étaient allées chez Bennygan's avant pis y nous avait dit? que $y$ avait une discothèque extraordinaire sur la première euh rue je pense, la premiẗre AvenUe $\mathrm{A} / \mathbf{y}$ (RESP .) euh pis IA $\%$ moi j'me suis, j'ai $z$ pis ta = MC dit: «c'est pratiquement» j'ai dit: NC «c'est proche $d E d E^{B} / \Rightarrow$ t.t...C'EST: proche de Coconut Grove pis tout ce coin-là là, t'sais t'es vraiment dans IE dOwntOwn euh ${ }^{C} / \rightarrow$ MIAm!. $/$ i $-D$ -

F-10-06 ÇA fAIT quEA $\| \Rightarrow \mathbb{A}^{\mathrm{B} /}$ \& ON pArt 68 syllabes IES trols, ${ }^{C} / \pi$ pls rendu à peu près à la IENC quatorzième euh rUe, jE pENse, ${ }^{D} /$. ON étaient arrétées à UN fEU roUge, ${ }^{E}$ i. Wis y avAlT plein d'autos et pis là tu? vois une bANde dE gARS ${ }^{F} /$ ArrlvER, G II y t'sAIS les petits débrouillards $H$ 


\section{G II t'sA'S les petits débrouillards \% \%ugement de valour cinquante qui décident qu'y ont besoin d'Une vomure.}

$H$ partant a la place de F. 


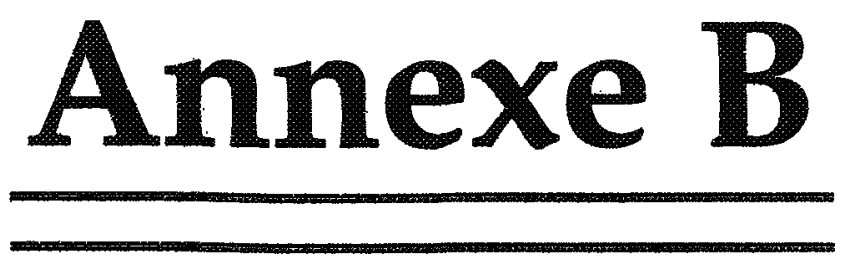

\section{Table de données " générale » H et F}


Table des données du locuteur $F$

\begin{tabular}{|c|c|c|c|c|c|c|c|c|c|c|c|c|c|c|}
\hline & & & & & Fo & $m p a$ on & of cheste & $\%$ de & variati & tion & & & & \\
\hline & octh & $F 0 \mathrm{moy}$ & Fomgy & onome & $\ln \phi t$ & $\min$ per & $\tan 6$ & \begin{tabular}{|l|l}
$A-P$ & \\
\end{tabular} & \begin{tabular}{|l|l|}
$A-F$ & $F$
\end{tabular} & P-F & crienta & $\ln 60$ & lnto & var \\
\hline Anondes: & grance & enonget & Louf & $\mathrm{FO}$ & $\operatorname{mog} t$ & moy : & ponutitin & & & & 58 & percuse & voy & dt \\
\hline 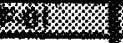 & & & & & & & & & & & & & & \\
\hline $804-01$ & 189 & 159,3 & & $t$ & & & & -43 & 34 & 136 & & & & 14,93 \\
\hline$F-0,-\infty 2$ & 59 & 138,8 & & $E$ & & & & -8 & -69 & -59 & & $x$ & & $-15,62$ \\
\hline$F-01.08$ & 156 & 162,5 & & $E$ & & & & 96 & 1 & -13 & & & & $-2,39$ \\
\hline Fot-as & 230 & 214,3 & & $t$ & & & & -16 & -2 & 17 & & $\lambda$ & +2 & 2,67 \\
\hline $7=01-65$ & 233 & 189,5 & & + & & & & -2 & 41 & 45 & & 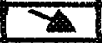 & & 6,42 \\
\hline$F=01-06$ & 263 & 218,5 & & + & & & & 19 & 34 & 13 & & & & 2,1 \\
\hline F-01-07 & 294 & 240 & & + & & & & 4 & 41 & 35 & & $\lambda$ & & 5,28 \\
\hline Fol-o & 233 & 213,8 & & \pm & & & & -30 & -7 & 32 & & & & 4,87 \\
\hline$=01-\infty$ & 236 & 232 & & $E$ & & & & 7 & 18 & 10 & & & & 1,7 \\
\hline$E-01-10$ & 179 & 186 & & $E$ & & & & -12 & -7 & 6 & 3] & 8 & & \\
\hline$F=01-11$ & 76 & 166,5 & & $E$ & & & & -27 & -67 & -55 & & 8 & & $-13,8$ \\
\hline$F-01-12$ & 245 & 224 & & 4 & & & & $-0,4$ & 7 & 8 & & $\lambda$ & & 1,33 \\
\hline $\mathrm{Fo1-13}$ & 101 & 158 & & $E$ & & & & -8 & 14 & 24 & & $x$ & & 3,79 \\
\hline Failit & 76 & 142,5 & 188 & - & - & $=$ & & -12 & -54 & -48 & & & & $-11,2$ \\
\hline ToTA: & & & & & & & & & & & & & & \\
\hline$<<$ & & & & 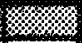 & & & & 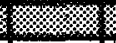 & 8 & 98 & & & ঝ & স৪। \\
\hline Fos of & 175 & 120,3 & & $\mp$ & & & & -1 & 101 & 103 & & & & 12,34 \\
\hline 80202 & 227 & 218,8 & & $\equiv$ & & & & $-15 \mid$ & 23 & 43 & & & & 6,18 \\
\hline $5-02-60$ & 168 & 160,8 & & + & & & & -3 & 12 & 15 & & $x$ & & 6,42 \\
\hline $5-02-\alpha$ & 161 & 174 & & $E$ & & & & 이 & -2 & -2 & & & +4 & $-0,32$ \\
\hline$=-02-08$ & 103 & 146 & & $=$ & & & & of & -46 & -46 & & 2 & & $-10,5$ \\
\hline$F-02-06$ & 159 & 187,3 & & - & & & & -9 & -35 & -29 & & $x$ & +1 & $-5,88$ \\
\hline $\mathrm{E}-08-07$ & 179 & 178,5 & 172,4 & $E$ & + & to & & -201 & -15 & & & &.+2 & \\
\hline IOTAL & & & & & & & & & & & & & 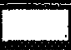 & \\
\hline H x x & & & & 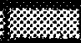 & & & & & ; & & & & & \\
\hline E-00.01 & 222 & 147,3 & & \pm & & & & -23 & 158 & 164 & & $\underline{x}$ & & 16,88 \\
\hline Fos.02 & 162 & 172 & & - & & & & -15 & -16 & -1 & & & & $-0,21$ \\
\hline$=-\infty-\infty$ & 204 & 182,3 & & $t$ & & & & -18 & 16 & 41 & & $\bar{T}$ & & 5,93 \\
\hline$F-0,04$ & 196 & 175 & 169,1 & $1+$ & + & + & & -9 & 23 & 35 & & & & 5,24 \\
\hline TOTAL & & & & & & & & & & & & & & \\
\hline 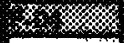 & & & & $1 \times$ & & & & & & & & & & \\
\hline E-0401 & 60 & 116,5 & & $E$ & & & & 83 & -22 & -57 & & 2 & & $-14,8$ \\
\hline$F 0402$ & 152 & 164,8 & & $\leftarrow$ & & & & -2 & -7 & -6 & & 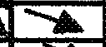 & +1 & -1 \\
\hline $7-04-\infty$ & 213 & 190,8 & & \pm & & & & 49 & -37 & -8 & & $\pi$ & & $-1,48$ \\
\hline Fomat & 111 & 188,5 & & - & & & & -69 & -60 & 28 & & 8 & & 4,23 \\
\hline Fos-06 & 65 & 111 & & $E$ & & & & -5 & -61 & -59 & & $x$ & & $-15,4$ \\
\hline F-04.0s & 96 & 148,3 & & $E$ & & & & -5 & 10 & 15 & & $\lambda$ & & 2,46 \\
\hline Fol-a: & 240 & 171,3 & & $F$ & & & & 4 & 32 & 27 & & & & 4,15 \\
\hline$-04-00$ & 193 & 139 & & + & & & & -11 & 110 & 135 & & $\bar{\lambda}$ & & 14,87 \\
\hline Fose & 2081 & 1159,3 & 156,9 & 14 & $t$ & + & 5 & 49 & -11 & 117 & & $\bar{x}$ & & 13,43 \\
\hline TOTAL & & & & 1 & & & & & & & & & & \\
\hline$\gamma$ & & & & & & & & & & & & & & (1. \\
\hline$F-05-00$ & 179 & 193 & & $E$ & & & 2 & -8 & -14 & -7 & 7 & $\nabla$ & & $-1,22$ \\
\hline F-05-01: & 164 & 171,8 & & $E$ & & & $\$$ & 0,6 & 5 & 4 & -1 & & & 0,76 \\
\hline$F-05-02$ & 208 & 145 & & $t$ & & & -7 & 3 & 104 & 88 & $=$ & $\rightarrow$ & d & 11,87 \\
\hline $6-06-\infty 8$ & 209 & 173,7 & & + & & & $\sqrt{2}$ & -8 & 31 & 42 & $\nabla$ & $\mathbf{8}$ & & 6,11 \\
\hline$F-05-04$ & 147 & 140,5 & & \pm & & & 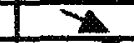 & -18 & -21 & -3 & 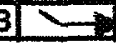 & 7 & & $-0,58$ \\
\hline
\end{tabular}




\begin{tabular}{|c|c|c|c|c|c|c|c|c|c|c|c|c|c|c|}
\hline F-08-05 & 182 & 183,5 & & $\equiv$ & & & & -15 & -2 & 14 & & 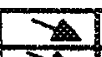 & & 2,35 \\
\hline$-05-06$ & 179 & 162,8 & & + & & & & -13 & 6 & 22 & & $\Delta$ & & 3,42 \\
\hline F.06-0? & 185 & 171,8 & & \pm & & & & -21 & 6 & 34 & & 8 & & 5,09 \\
\hline$F-05-08$ & 149 & 148 & & $\equiv$ & & & & -43 & of & 94 & & & & 11,47 \\
\hline $\mathrm{F}-05-00$ & 156 & \begin{tabular}{l|l}
158 & 1 \\
\end{tabular} & 164,6 & $\equiv$ & E & - & 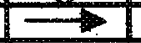 & -3 & -1 & 5 & $\overline{7}$ & &.+2 & 0,8 \\
\hline \multicolumn{15}{|l|}{ TOTAL } \\
\hline \multicolumn{15}{|l|}{ 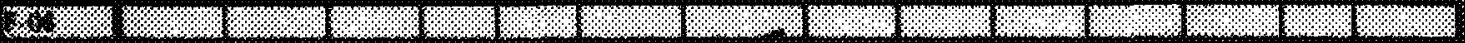 } \\
\hline$F=06-0\}$ & 169 & 157,3 & & \pm & & & & -6 & 8 & $-15 \mid$ & & 7 & & 2,42 \\
\hline$F-06-02$ & 168 & 164,8 & & $\equiv$ & & & & -5 & $-0,6$ & 4 & & $x$ & & 0,75 \\
\hline$F-06-08$ & 80 & 149 & & $=$ & & & & 2) & -53 & -54 & & & & $-13,6$ \\
\hline$-06-04$ & 232 & 171,5 & & + & & & -7 & 4 & 39 & 34 & & & & 5,1 \\
\hline$=-06-06$ & 213 & 212,8 & & $E$ & & & & -9 & -13 & -4 & & $\Delta$ & & $-0,72$ \\
\hline$=-\infty 6-\infty 6$ & 179 & 201,8 & & $E$ & & & & -10 & 2 & 13 & & & & 2,17 \\
\hline -06-07 & 238 & 180,3 & & + & & & & 4 & 47 & 41 & & & & 5,95 \\
\hline 5000 & 146 & 135,5 & 172,1 & 7 & - & - & 4 & -12 & 15| & -3 & & & & $-0,47$ \\
\hline \multicolumn{15}{|l|}{ fotal } \\
\hline \multicolumn{15}{|l|}{ 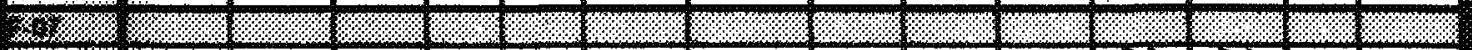 } \\
\hline $70702 /$ & 80 & 140,5 & & - & & & & -9 & -53 & -49 & & $\Delta$ & & $-11,6$ \\
\hline $207-08$ & 200 & 188 & & + & & & & -18 & 6 & 29 & & & & 4,43 \\
\hline $507-04$ & 86 & 173 & & $E$ & & & & -9 & 15 & 26 & & 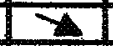 & & 4,06 \\
\hline $7-07.05$ & 69 & 144,5 & & 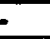 & & & $x$ & 16 & -43 & -33 & 8 & $\mathbf{x}$ & & $-6,88$ \\
\hline$E-07.06$ & 170 & 164,5 & & $\equiv$ & & & & 0 & 2 & 2 & $\Rightarrow$ & $\mathbf{z}$ & & 0,31 \\
\hline$=07.07$ & 222 & 175,5 & & \pm & & & & 4 & 42 & 37 & & & & 5,47 \\
\hline E.07.68 & 97 & 115,3 & 157,3 & - & $=$ & $E$ & $\overline{7}$ & 0) & 8 & 8 & & & & 1,35 \\
\hline \multicolumn{15}{|l|}{ Forat } \\
\hline \multicolumn{15}{|l|}{ 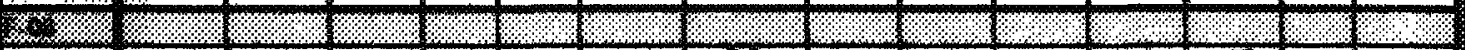 } \\
\hline $200-01$ & 204 & 215,5 & & 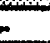 & & & 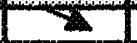 & -13 & -20 & -9 & & & & $-1,55$ \\
\hline Folo2 & 219 & 226,8 & & $\equiv$ & & & & 201 & -16 & -4 & & & & $-0,62$ \\
\hline $7-00-\infty$ & 200 & 219 & & $E$ & & & & -23 & -26 & $-4 \mid$ & & & & $-0,76$ \\
\hline $\mathrm{F}-\cos -\mathrm{os}^{2}$ & 193 & 185,3 & & $\equiv$ & & & & -4 & 4 & 81 & & $x$ & & 1,41 \\
\hline$F-00-\infty$ & 244 & 233 & & \pm & & & & 0 & 10 & 10 & & 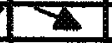 & $2 \times m$ & 1,64 \\
\hline 50806 & 172 & 177,5 & 207,4 & $E$ & - & $E$ & & -14 & -14 & $-0,6$ & & & & $-0,1$ \\
\hline \multicolumn{15}{|c|}{ Toral } \\
\hline \multicolumn{15}{|c|}{ X } \\
\hline-0901 & 244 & 230,5 & & + & & & & of & 12 & $\sqrt{2}$ & & & & 2,04 \\
\hline$F-00 \cdot 02$ & 182 & 204 & & $E$ & & & & 2 & -13 & -15 & & 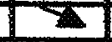 & & $-2,73$ \\
\hline$F-00-03$ & 213 & 241,8 & & $E$ & & & & -25 & -32 & -9 & & 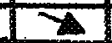 & & $-1,56$ \\
\hline$F-0 \operatorname{0os}$ & 245 & 231,8 & & $t$ & & & & -43 & -10 & $59 \mid$ & & \pm & & 8,07 \\
\hline $7-0005$ & 227 & 216,8 & & $t$ & & & & 44 & 34 & -7 & $\nabla$ & $\overline{7}$ & & $-1,25$ \\
\hline$E-00-\infty$ & 182 & 212,8 & 222,2 & $E$ & $E$ & - & & 7 & 21 & 9 & & 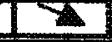 & & 1,49 \\
\hline \multicolumn{15}{|l|}{ TOTAL } \\
\hline \multicolumn{15}{|l|}{ אu } \\
\hline$F=10-01$ & 270 & 270 & & $E$ & & & $\rightarrow$ & 0 & 0 & of & & $\pi$ & & - \\
\hline$F-10-02$ & 156 & 177,5 & & $E$ & & & 5 & -24 & -25 & -2 & & $\Delta$ & +1 & $-0,33$ \\
\hline$F-10-08$ & 167 & 197,8 & & $E$ & & & 75 & 24 & -21 & -37 & 7 & $x$ &.+2 & $-7,89$ \\
\hline$F=10-04$ & 213 & 190,3 & & + & & & $\Rightarrow$ & 43 & 40 & $-2 \mid$ & $F$ & $\pi$ & & $-0,32$ \\
\hline$F-10-05$ & 238 & 187 & & + & & & 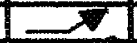 & -3 & 481 & 53 & & 5 & & 7,34 \\
\hline$F-10-06$ & 217 & 199,8 & 194,8 & + & + & $t$ & $\Delta$ & -22 & -3 & 25 & 17 & $x$ & & 3,94 \\
\hline TOTAL & & & & & & & & & & & & & & \\
\hline TOTAL & & & & & & & & & & & & & & \\
\hline
\end{tabular}


Table des données du locuteur $H$

\begin{tabular}{|c|c|c|c|c|c|c|c|c|c|c|c|c|c|c|}
\hline & & & & & 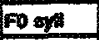 & mor fo & Chere & $\%$ dev & variato & & & & & \\
\hline & 50 in $1 M$ & MCy Fo & Fomoy & onenta & $\ln p$ & tera on pre & $\operatorname{anc} 6$ & A-P & $A-F$ & PF & crlenta & unto & into & var. \\
\hline fronods. & monote & anonos Io & bour & po & moy sour & $F 0$ & panuesten & & & & $5 \%$ & percues & koy & dt \\
\hline \multicolumn{15}{|l|}{ x } \\
\hline H-01.01 & 59 & 97,75 & & $=$ & & & & .26 & -54 & -37 & & & & $-8,09$ \\
\hline H-01.02 & 99 & 110,5 & & 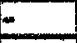 & & & & $-0,8$ & .14 & -13 & 3 & & & $-2,45$ \\
\hline H-01-08 & 175 & 134 & & + & & & & 21 & 97 & 62 & & &.+2 & 8,38 \\
\hline $4-01-04$ & 82 & 85,5 & 114,08 & $E$ & 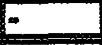 & $=$ & & 0 & 8 & -8 & & & $2 x m+2$ & $-1,42$ \\
\hline \multicolumn{15}{|l|}{ Toral: } \\
\hline \multicolumn{15}{|l|}{$8<$} \\
\hline H-oi-ot & 128 & 111,8 & & $\rightleftarrows$ & & & & -11 & 19 & 33 & & & & 5 \\
\hline 4-01-02 & 111 & 112,5 & & $=$ & & & & -36 & -21 & 23 & & & & 3,64 \\
\hline $401-08$ & 121 & 104 & 112,13 & + & + & 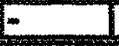 & & -2 & 23 & 261 & & & & 4,02 \\
\hline \multicolumn{15}{|l|}{ Total } \\
\hline \multicolumn{15}{|l|}{$3 x$} \\
\hline H-CB-01 & 154 & 152 & & $E$ & & & & 10 & -3 & -12 & & & & $-2,22$ \\
\hline 1.03-08 & 110 & 131,5 & & - & & & & -27 & -29 & -2 & & & & $-5,85$ \\
\hline $1+03-03$ & 103 & 133,8 & & 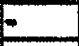 & & & & -11 & -11 & 0 & & & & 0 \\
\hline $4-02-04$ & 152 & 122,5 & & 4 & & & & 2 & 32 & 30 & & & & 4,55 \\
\hline 1-03-05 & 137 & 147 & & $E$ & & & & -9 & -22 & -14 & & & & $-2,59$ \\
\hline $4-020$ & 123 & 126 & 137,35 & $E$ & 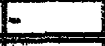 & - & & 0 & 0 & 0 & & & $3 x \mathrm{xm}$ & 0 \\
\hline \multicolumn{15}{|l|}{ DORA: } \\
\hline$x_{x}$ & & & & & & & & . & & $\%$ & & & & \\
\hline $4-0401$ & 106 & 106 & & $\equiv$ & & & & 0 & o & 0 & & & & 0 \\
\hline $1-0402$ & 114 & 80,5 & & 4 & & & & 56 & 216 & 104 & & & & 12,36 \\
\hline 40400 & 107 & 110 & & $=$ & & & & -13 & -3 & 11 & & & & 1,88 \\
\hline $4-0404$ & 118 & 117,3 & & $E$ & & & & -36 & -21 & 23 & & & & 3,85 \\
\hline $4-04-65$ & 132 & 127,3 & & $E$ & & & & -13 & -13 & 0 & & & & 0 \\
\hline 4.0403 & 108 & 101 & & + & & & & -14 & 5 & 21 & & & & 3,36 \\
\hline $\mathrm{H}-040$ & 136 & 115,5 & & + & & & & $-0,9$ & 25 & 54 & & & & 4 \\
\hline $4-04-08$ & 120 & 112,5 & & 4 & & & & -14 & 7 & 25 & & & & 3,88 \\
\hline $4-340$ & 123 & 124 & & $=$ & & & & 15 & 6 & -13 & & & & $-1,36$ \\
\hline H-04 10 & 112 & 123,3 & & - & & & & -36 & -29 & 11 & & & & 1,8 \\
\hline 4-04-11. & 112 & 114 & & $E$ & & & & -12 & 5 & 18 & & & & 3,04 \\
\hline 3-04-12 & 115 & 112.5 & & $\equiv$ & & & & -4 & 10 & 15 & & 2 & & 2,43 \\
\hline 10418 & 110 & 110 & & $E$ & & & & 9 & 8 & 0 & & 2 & & 0 \\
\hline $\mathrm{H}-04-14$ & 92 & 96 & & $E$ & & & 8 & -7 & -9 & -2 & & 2 & & $-0,37$ \\
\hline H-O 15 & 94 & 127 & & $=$ & & & & .21 & -37 & -20 & & & & $-3,95$ \\
\hline 4-04 16 & 108 & 114,3 & 111,77 & $E$ & - & + & & -14 & -11 & 3 & & & & 0,5 \\
\hline \multicolumn{15}{|l|}{ TOTAL } \\
\hline 46 & & & & & & & & & & & & & & \\
\hline $4-05-01$ & 122 & 110,5 & & + & & & - & 21 & 27 & 5 & & & & 0,88 \\
\hline $4-06-02$ & 94 & 104,3 & & $E$ & & & & -10 & -10 & 0 & & & & 0 \\
\hline H-05-03 & 108 & 104 & & $=$ & & & & -24 & .12 & 16 & & 2 & & 2,6 \\
\hline $4-0.04$ & 104 & 111 & & $E$ & & & 12 & -21 & -19 & 3 & 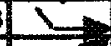 & 2 & & 0,51 \\
\hline $4-05-05$ & 102 & 101,3 & & $E$ & & & & -25 & -17 & 9 & & 2 & & 1,78 \\
\hline $1-05-05$ & 115 & 108 & 106,2 & 16 & + & $t$ & & -6 & -8 & 14 & & 2 & & 2,26 \\
\hline Toral & & & & & & & & & & & & & & \\
\hline
\end{tabular}




\begin{tabular}{|c|c|c|c|c|c|c|c|c|c|c|c|c|c|c|}
\hline & & & & & FO syl & Moy Fo & Chate & & & & & & & \\
\hline & Fo fin & Moy Fo & Fo moy & orient: & fin $p^{\prime}$ & ono.tin & antepen & $\%$ der & variation & & otenta & Into & $\operatorname{linfo}$ & var. \\
\hline 10 & Shond & énoncét & tour & Fo & moy tou & $\mathrm{p} / \mathrm{FO}$ tou & firzade & A-P & A-F $\mid F$ & P.F & $5 \%$ & peraves & noy & Idt \\
\hline$H-06-01$ & 154 & 141,8 & & \pm & & & & -16 & 3 & 23 & & & & 3,62 \\
\hline $1.06-02$ & 84 & 116,3 & & $F$ & & & & 28 & -26 & -42 & & 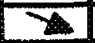 & & $-9,48$ \\
\hline 1.08 .03 & 122 & 107,3 & & $F$ & & & & -11 & 17 & 28 & & $x$ & & 4,35 \\
\hline $4.06-04$ & 100 & 105,3 & & $E$ & & & & 0,9 & -71 & -7 & & $x$ & & $-1,34$ \\
\hline H-06-05 & 103 & 104,3 & & $E$ & & & & 4 & -6 & -2 & $7 \pi$ & $\Phi$ & & $-0,33$ \\
\hline $1+00-06$ & 147 & 119,5 & & + & & & 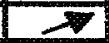 & 58 & 65 & 4 & $\$$ & & & 0,72 \\
\hline $4-08-02$ & 128 & 112 & & + & & & & 19 & 35 & 13 & & 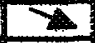 & & 2,17 \\
\hline $406-08$ & 97 & 102 & 115,14 & $E$ & & - & & 10 & -1 & -10 & & \pm & +1 & $-1,87$ \\
\hline forat. & & & & & & & & & & $-\infty$ & & & 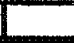 & \\
\hline 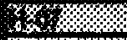 & & & & & & & & & & & & & & \\
\hline 900701 & 111 & 111 & & $=$ & & & & 0 & of & 0 & & $A$ & & 0 \\
\hline 407.02 & 119 & 119 & & $E$ & & & & of & of & o & & $\Delta$ & $3 x \mathrm{~m}$ & 0 \\
\hline 4.07 .03 & 911 & 111,8 & & - & & & & -7 & -5 & 2 & & $\$$ & & 0,32 \\
\hline 40704 & 120 & 117 & & $\equiv$ & & & & -14 & 10 & 28 & & & & 4,24 \\
\hline 407.65 & 94 & 101 & & $E$ & & & & -7 & -10 & -3 & & \pm & & $-1,76$ \\
\hline 40706 & 110 & 109 & & $=$ & & & & -13 & -2 & 12 & & $x$ & & 2,01 \\
\hline 4-07-07 & 115 & 105 & & + & & & & -8 & 14 & 24 & & $y$ & & 3,69 \\
\hline 10700 & 98 & 97,25 & 110,54 & $E$ & - & $=$ & & -4 & -3 & 1 & & 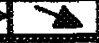 & & 0,18 \\
\hline torkl & & & & & & & & 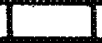 & 1 & & & & & \\
\hline $3 x-3$ & & & & & & & & & & & & & & ڤ\% \\
\hline $4+00-01$ & 164 & 131,3 & & $\omega$ & & & & 31 & 47 & 13 & & & & 2,14 \\
\hline 100 & 112 & 110,8 & & $E$ & & & & -25 & -16 & 12 & & & & 1,97 \\
\hline $4-00.03$ & 116 & 109,8 & & + & & & & -14 & 6 & 23 & & $\bar{x}$ & & 3,65 \\
\hline $300-a$ & 102 & 107,5 & & $E$ & & & $\mathbf{\Delta}$ & -4 & -11 & -7 & & $\bar{x}$ & +2 & $-1,31$ \\
\hline $400-0$ & 100 & 99 & & $E$ & & & & -6 & 0 & 6 & & 5 & & 1,07 \\
\hline Hos & 104 & 103 & & $E$ & & & & 2 & 3 & 0,9 & & 2 & & 0.17 \\
\hline $1+00.07$ & 116 & 103,5 & & + & & & & -8 & 13 & 22 & & $x$ & & 3,47 \\
\hline$H-\infty 6$ & 57 & 88,75 & & $E$ & & & & -2 & -45 & -43 & & $x$ & & $-9,77$ \\
\hline 40000 & 118 & 116,5 & & $E$ & & & & 0 & 3 & 3 & & $x$ & & 0,45 \\
\hline Hos:10 & 90 & 110,8 & & $E$ & & & & -10 & -30 & -22 & & & & $-4,41$ \\
\hline$H-\infty 6)$ & 133 & 106,8 & 108,08 & + & 4 & $E$ & & -12 & 27 & 43 & & $x$ & & 6,21 \\
\hline Total & & & & & & & & & & & & & & \\
\hline $\mathrm{Jg}$ & & & & & & & & & & & & & & 3 \\
\hline $4+\infty 001$ & 132 & 117,5 & & + & & & & -15 & -2 & 15 & & & & 2,4 \\
\hline$H-0,02$ & 154 & 116,8 & & \pm & & & & -21 & 33 & 67 & & 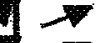 & & 8,95 \\
\hline$H-0000$ & 121 & 116,3 & & + & & & & -29 & -5 & 33 & & $\rightarrow$ & & 4,95 \\
\hline 4000 & 98 & 117,5 & & $E$ & & & & -27 & -30 & -5 & & $\pi$ & +2 & $-0,88$ \\
\hline $400-05$ & 91 & 125,3 & 117 & & $E$ & $t$ & & 17 & -9 & -24 & & & & $-4,66$ \\
\hline TOTAL: & & & & & & & & & & & & & & \\
\hline $3 x$ & & & & & & & & & & & & & & \\
\hline $4-10-01$ & 133 & 154,8 & & $E$ & & & $z$ & -34 & -35 & -1 & 1 & 4 & & $-0,26$ \\
\hline 410,02 & 113 & 102 & & $t$ & & & 2 & -8 & 8 & 16 & & 3 & & 2,65 \\
\hline $4-10-08$ & 125 & 148 & & $E$ & & & & 26 & 0 & -20 & & 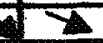 & & $-3,96$ \\
\hline $4+10-01$ & 100 & 99,5 & & $=$ & & & 4 & -10 & -11 & 10 & & 15 & & 1,64 \\
\hline $4-10-05$ & 106 & 88,5 & & \pm & & & 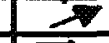 & 6 & 18 & 12 & $\geqslant$ & $\Rightarrow$ & & 1,9 \\
\hline $\mathrm{H}-10-06$ & 94 & 107,3 & & $E$ & & & 4 & 0 & -14 & -14 & & 17 & $1+1$ & $-0,9$ \\
\hline
\end{tabular}




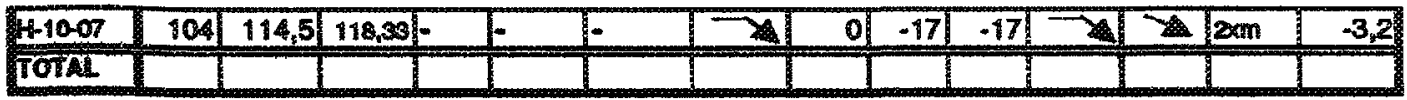

Logenda;

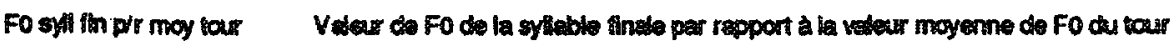

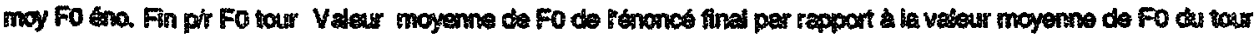

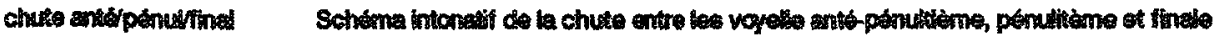
A-P

A-F

P.F

crienta $3 \%$

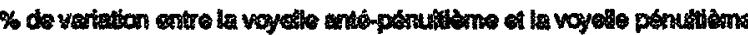

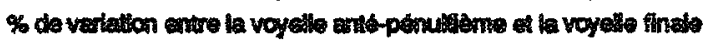

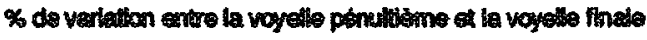

into perçuse

.41

$+2$

.+3

$+4$

$2 \times m$

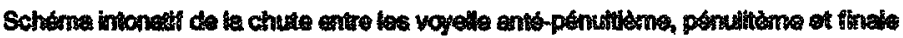

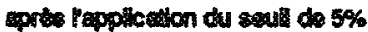

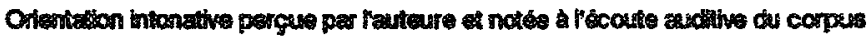

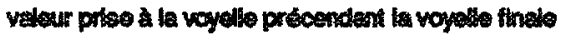

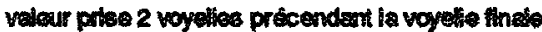

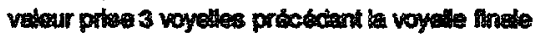

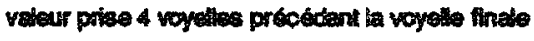
deux tois la mame valeur

$3 \times \mathrm{m}$ wois fol la mono valour




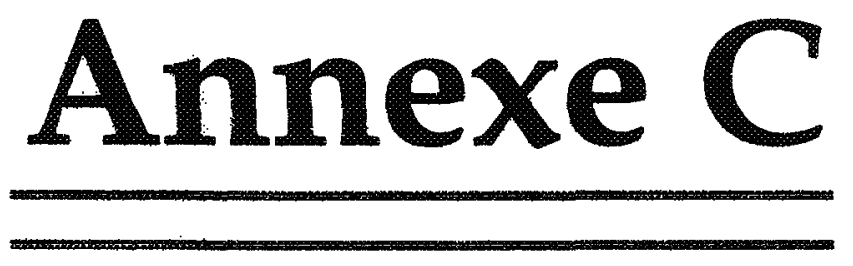

\section{Table de données « intensité » $H$ et $F$}




\begin{tabular}{|c|c|c|c|}
\hline & ds voy. & moy. & \\
\hline & finale Ty & voy ina & ale \\
\hline Gones & enonce & $28 \operatorname{tin} 10$ & Quts \\
\hline 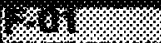 & & & \\
\hline$F-01-01$ & 59,90 & & \\
\hline F-01-02 & 59,68 & & \\
\hline$F-01-03$ & 65,99 & & \\
\hline$F-01.04$ & 84,62 & & \\
\hline F-01-05 & 65,23 & & \\
\hline$F-01-06$ & 51,87 & & \\
\hline$F-01-07$ & 68,75 & & \\
\hline$F-01-08$ & 72,76 & & \\
\hline$F-01-08$ & 69,89 & & \\
\hline$F-01-10$ & 52,74 & & \\
\hline$F-01-11$ & 61,30 & & \\
\hline$F-01-12$ & 71,22 & & \\
\hline$F-01-13$ & 59,80 & 63,37 & \\
\hline$F=01-14$ & 53,75 & & \\
\hline IOIAL & & & \\
\hline 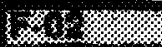 & & & \\
\hline F02-01 & 63,18 & & \\
\hline$F-02-02$ & 76,64 & & \\
\hline$F-02-03$ & 66,76 & & \\
\hline F-02-04 & 58.84 & & \\
\hline$F-02-05$ & 66,68 & & \\
\hline$F-02-06$ & 62,53 & 65,77 & \\
\hline 50207 & 72,73 & & \\
\hline TothL & & & \\
\hline (6) & & & \\
\hline F-03401 & 60,53 & & \\
\hline$F-03-62$ & 59,35 & & \\
\hline$F-03-03$ & 75,00 & 64,96 & \\
\hline$F-0004$ & 74,20 & & \\
\hline rovat & & & \\
\hline 女为 & & & \\
\hline$F-0401$ & 52,12 & & \\
\hline$F-04-02$ & 62,33 & & \\
\hline$F-04 \times 03$ & 54,34 & & \\
\hline$F-04-04$ & 59,61 & & \\
\hline$F-04-05$ & 57,03 & & \\
\hline$F-04-06$ & 59,86 & & \\
\hline F-04-07 & 64,26 & & \\
\hline $\mathrm{F}-04-08$ & 57,38 & 58,37 & \\
\hline$F-0400$ & 59,71 & & \\
\hline TOLA & & & \\
\hline 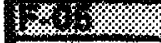 & & & \\
\hline F-05-00 & 58,35 & & \\
\hline$F-05-01$ & 57,33 & & \\
\hline$F-05-02$ & 60,83 & & \\
\hline F-05-03 & 61,25 & & \\
\hline
\end{tabular}

\begin{tabular}{|c|c|c|c|}
\hline & ds voy. In & moy. vo & \\
\hline & Iin. Enolt & (inale & \\
\hline nonods & & ss fin tol & \\
\hline 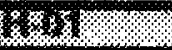 & & & \\
\hline H-01-01 & 58,52 & & \\
\hline H-01-02 & 66,44 & & \\
\hline H-01-03 & 68,96 & 64,64 & \\
\hline H-01-04 & 66,28 & & \\
\hline TOTAL & & & \\
\hline 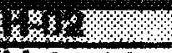 & & & \\
\hline H-01-01 & 65,72 & & \\
\hline $\mathrm{H}-01-02$ & 60,71 & 63,22 & \\
\hline $\mathrm{H}-01-03$ & $3 / 4 \%$ & & \\
\hline TOTAL & & & \\
\hline 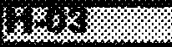 & & & \\
\hline 11-03-01 & 73,71 & & \\
\hline H-03-02 & 68,63 & & \\
\hline $1+03-03$ & 71,33 & & \\
\hline H-03-04 & 67,17 & & \\
\hline H-03-05 & 57,01 & 67,57 & \\
\hline H-03-06 & 66,14 & & \\
\hline TOTAL & & & \\
\hline$\left[\begin{array}{l}k \\
3\end{array}\right.$ & & & \\
\hline $\mathrm{H}-04-01$ & 56,17 & & \\
\hline H-04-02 & 59,45 & & \\
\hline $\mathrm{H}-04-03$ & 62,59 & & \\
\hline H-04-04 & 66,56 & & \\
\hline H-04-05 & 67,5 & & \\
\hline H-04-06 & 62,72 & & \\
\hline H-04-07 & 68,71 & & \\
\hline H-04-08 & 59,13 & & \\
\hline H-04-09 & 71,92 & & \\
\hline 140410 & 66,68 & & \\
\hline H-04-11 & 63,3 & & \\
\hline $\mathrm{H}-04-12$ & 62,13 & & \\
\hline $\mathrm{H}-04-13$ & 68,98 & & \\
\hline $\mathrm{H}-04-14$ & 58,96 & & \\
\hline H-04-15 & 65,81 & 64,04 & \\
\hline H-04-16 & 63,52 & & \\
\hline TOTAL & & & \\
\hline 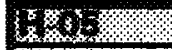 & & & \\
\hline H-05-01 & 61,2 & & \\
\hline H-05-02 & 60,49 & & \\
\hline H-05-03 & 66,21 & & \\
\hline H.05-04 & 53,37 & & \\
\hline H-05-05 & 59,55 & 60,16 & \\
\hline H-05-06 & 63,87 & & \\
\hline TOTAL & & & \\
\hline 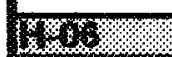 & & & \\
\hline
\end{tabular}




\begin{tabular}{|c|c|c|c|}
\hline$F-05-04$ & 57,021 & & \\
\hline F-05-05 & 71,27 & & \\
\hline$F=05-0.6$ & 70,75 & & \\
\hline$F-05-07$ & 58,92 & & \\
\hline$F-05-08$ & 50,14 & 60,65 & \\
\hline$F-05-09$ & 52,72 & & \\
\hline \multicolumn{4}{|l|}{ TOK 1} \\
\hline \multicolumn{4}{|l|}{ - } \\
\hline$F-06-01$ & 62,04 & & \\
\hline$F-06-02$ & 56,01 & & \\
\hline$F-06-03$ & 60,15 & & \\
\hline$F-06-04$ & 70,18 & & \\
\hline$F-06-05$ & 69,43 & & \\
\hline$F-06-08$ & 64,84 & & \\
\hline F-0607 & 59.77 & 63,20 & \\
\hline$F-06-08$ & $8 \times 1$ & & \\
\hline \multicolumn{4}{|l|}{ rold: } \\
\hline \multicolumn{4}{|l|}{$6 \%$} \\
\hline F.07-02 & 56,61 & & \\
\hline$F-07.03$ & 61,92 & & \\
\hline$F-07-04$ & 57,16 & & \\
\hline$F-07-05$ & 51,35 & & \\
\hline$F-07-06$ & 63,16 & & \\
\hline $\mathrm{F}-07-07$ & 59,49 & 58,28 & \\
\hline$F-070$ & 55,67 & & \\
\hline \multicolumn{4}{|l|}{ TOVIS } \\
\hline \multicolumn{4}{|l|}{4 - } \\
\hline$F-0001$ & 65,93 & & \\
\hline $\mathrm{F} .08 .02$ & 77,75 & & \\
\hline$F-0800$ & 70,66 & & \\
\hline F.00-0\% & 60,17 & & \\
\hline F-080s & 68,92 & 68,69 & \\
\hline$F-00406$ & 65,66 & & \\
\hline \multicolumn{4}{|l|}{ ToW } \\
\hline \multicolumn{4}{|l|}{ S } \\
\hline F-0961 & 73,07 & & \\
\hline$F-09002$ & 66,14 & & \\
\hline $\mathrm{F}-090 \mathrm{~s}$ & 60,86 & & \\
\hline$F-0904$ & 63,35 & & \\
\hline$F-090$ & 65,94 & 65,87 & \\
\hline$F-0000$ & 65,60 & & \\
\hline \multicolumn{4}{|l|}{ TOLAL } \\
\hline \multicolumn{4}{|l|}{ 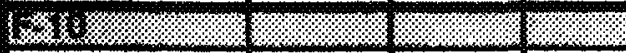 } \\
\hline F-10-01 & 67,56 & & \\
\hline$F-10-02$ & 66,10 & & \\
\hline$F-10-03$ & 58,81 & & \\
\hline$F-10-04$ & 74,70 & & \\
\hline$F-10-05$ & 71,68 & 67,77 & \\
\hline$F-10-06$ & 71,95 & & \\
\hline TOTAL & & & \\
\hline
\end{tabular}

\begin{tabular}{|c|c|c|c|}
\hline H-06-01 & 71,5 & & \\
\hline $\mathrm{H}-06-02$ & 60,36 & & \\
\hline H-06-03 & 62,67 & & \\
\hline 4-06-04 & 64,27 & & \\
\hline H-06-05 & 69,63 & & \\
\hline H-06-06 & 60,04 & & \\
\hline H-06-07 & 57,38 & 63,69 & \\
\hline A-06-08 & S6:Gy & & \\
\hline \multicolumn{4}{|l|}{ TOTAL } \\
\hline \multicolumn{4}{|l|}{ 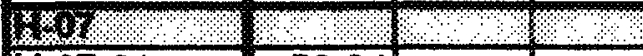 } \\
\hline H-07-01 & 58,94 & & \\
\hline $\mathrm{H}-07-02$ & 60,23 & & \\
\hline H-07-03 & 69,23 & & \\
\hline H-07-04 & 68,52 & & \\
\hline H-07-05 & 57,68 & & \\
\hline H-07-06 & 63,45 & & \\
\hline H-07-07 & 62,77 & 62,97 & \\
\hline H-07-08 & 60,99 & & \\
\hline \multicolumn{4}{|l|}{ TOTaL } \\
\hline \multicolumn{4}{|l|}{ 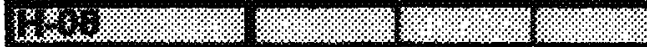 } \\
\hline H-08-01 & 74,28 & & \\
\hline H-08-02 & 63,64 & & \\
\hline H-08-03 & 57,31 & & \\
\hline H-08-04 & 63,12 & & \\
\hline H-08-05 & 50,49 & & \\
\hline H-08-06 & 50,25 & & \\
\hline H-08-07 & 56,06 & & \\
\hline H-08-08 & 54,83 & & \\
\hline H-08-09 & 60,15 & & \\
\hline $\mathrm{H}-08-10$ & 63,53 & 59,37 & \\
\hline H-08-11 & 54,64 & & \\
\hline \multicolumn{4}{|l|}{ TOTAL } \\
\hline \multicolumn{4}{|l|}{ 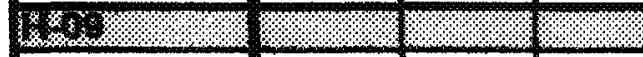 } \\
\hline H-09-01 & 64,15 & & \\
\hline H-09-02 & 59,3 & & \\
\hline H-09-03 & 60,92 & & \\
\hline $\mathrm{H}-09-04$ & 60,87 & 61,31 & \\
\hline H-09-05 & 60,88 & & \\
\hline \multicolumn{4}{|l|}{ TOTAL } \\
\hline \multicolumn{4}{|l|}{ 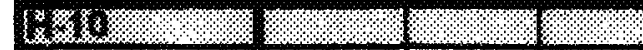 } \\
\hline H-10-01 & 60,31 & & \\
\hline H-10-02 & 59,05 & & \\
\hline $\mathrm{H}-10-03$ & 63,59 & & \\
\hline$H-10-04$ & 50,17 & & \\
\hline $\mathrm{H}-10-05$ & 58,8 & & \\
\hline $\mathrm{H}-10-06$ & 48,9 & 56,8 & \\
\hline $\mathrm{H}-10-07$ & 58,32 & & \\
\hline TOTAL & & & \\
\hline & & & \\
\hline
\end{tabular}




\begin{tabular}{|c|c|c|}
\hline & $\begin{array}{l}\text { valeur de la voyelle } \\
\text { finale de tour }\end{array}$ & $\begin{array}{l}\text { Valeur moyenne des } \\
\text { voyelles finales çénoncé } \\
\text { dans le tour correspondant }\end{array}$ \\
\hline Hot & 66,28 & 64,64 \\
\hline $\mathrm{H}-02$ & 51,26 & 63,22 \\
\hline $\mathrm{H}-03$ & 66,14 & 67,57 \\
\hline $\mathrm{H}-04$ & 63,52 & 64,04 \\
\hline $\mathrm{H}-05$ & 63,87 & 60,16 \\
\hline H-06 & 56,84 & 63,69 \\
\hline H-07 & 60,99 & 62,97 \\
\hline H.08 & 54,64 & 59,37 \\
\hline $\mathrm{H}-0 \mathrm{~g}$ & 60,88 & 61,31 \\
\hline$H-10$ & 58,32 & 56,8 \\
\hline F-01 & 53,75 & 63,37 \\
\hline F.02 & 72,73 & 65,77 \\
\hline $\mathrm{F}-03$ & 74,20 & 64,96 \\
\hline$F-04$ & 59,71 & 58,37 \\
\hline$F-05$ & 52,72 & 60,65 \\
\hline$F \cdot 06$ & 54,26 & 63,2 \\
\hline$F-07$ & 55,67 & 58,28 \\
\hline$F-08$ & 65,66 & 68,69 \\
\hline F-09 & 65,60 & 65,87 \\
\hline$F-10$ & 71,95 & 67,77 \\
\hline
\end{tabular}




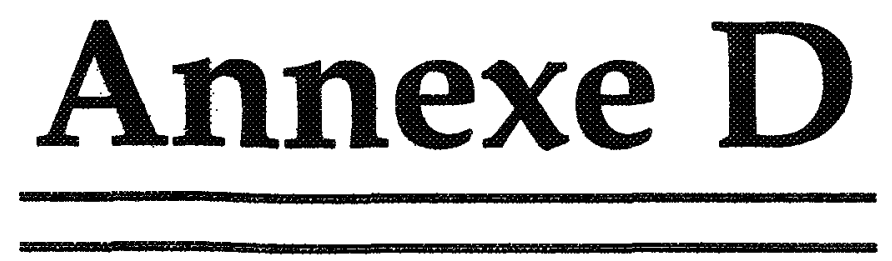

Table de données

«longueur en syllabes "

$H$ et $F$ 


\begin{tabular}{|c|c|c|}
\hline & no syl & Woyenne \\
\hline enonces: & & \\
\hline \multicolumn{3}{|l|}{ 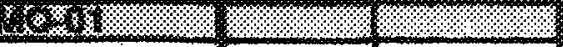 } \\
\hline \multirow{2}{*}{\multicolumn{3}{|c|}{$F-01-02$}} \\
\hline & 18 & \\
\hline$F-01-03$ & 5 & \\
\hline$F-01-04$ & 5 & \\
\hline$F-01-05$ & 15 & \\
\hline$F-01-06$ & 71 & \\
\hline$F-01-07$ & 3) & \\
\hline$F-01-08$ & 13 & \\
\hline F-01-09 & 26 & \\
\hline$-01-10$ & 18 & \\
\hline $5-01-11$ & 13 & \\
\hline$F=01-12$ & 8 & \\
\hline$F-01-13$ & 46 & 14,75 \\
\hline$F-01-14$ & 45 & \\
\hline \multicolumn{3}{|l|}{ TOTAL: } \\
\hline \multicolumn{3}{|l|}{ 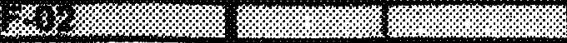 } \\
\hline$F-02-01$ & 401 & \\
\hline$F-0202$ & 53 & \\
\hline$F-02-03$ & 15 & \\
\hline$F-02-04$ & 10 & \\
\hline$F_{-02-05}$ & 2 & \\
\hline$F-02-06$ & 23 & 23,83 \\
\hline 10207 & 12 & \\
\hline \multicolumn{3}{|l|}{ WOALL } \\
\hline \multicolumn{3}{|l|}{ <x: } \\
\hline$F-03-01$ & 23 & \\
\hline$F-03-02$ & 11 & \\
\hline $\mathrm{F}-\mathbf{0 3}-0 \mathbf{0}$ & 40 & 24,67 \\
\hline$F-03-04$ & 541 & \\
\hline \multicolumn{3}{|l|}{ IOIAL } \\
\hline \multicolumn{3}{|l|}{ 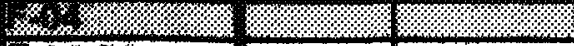 } \\
\hline F-04-0T & 13 & \\
\hline$F-04-02$ & 30 & \\
\hline$F-04-03$ & 44 & \\
\hline$F-04-04$ & 37 & \\
\hline$F-04-05$ & 25 & \\
\hline$F-04-06$ & 68 & \\
\hline F-04-07? & 54 & \\
\hline$F-04-08$ & 10 & 35,125 \\
\hline F-04-00 & 6 & \\
\hline \multicolumn{3}{|l|}{ TOTAL } \\
\hline \multicolumn{3}{|l|}{ 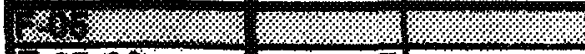 } \\
\hline$F-05-00$ & 7 & \\
\hline$F-05-01$ & 16 & \\
\hline$F-05-02$ & 8 & \\
\hline $\mathrm{F}-05-03$ & 61 & \\
\hline$F-05-04$ & 7 & 1 \\
\hline
\end{tabular}

\begin{tabular}{|c|c|c|}
\hline & nb syll & Moyenne \\
\hline troncas: & Snoncés & \\
\hline $6 \mathrm{x} / \mathrm{s} / \mathrm{s}$ & & \\
\hline H-01-01 & 34 & \\
\hline H-01-02 & 29 & \\
\hline H-01-03 & 49 & 37,33 \\
\hline H-01-04 & 4 & \\
\hline TOTAL & & \\
\hline 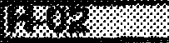 & & \\
\hline H-02-01 & 23 & \\
\hline H-02-02 & 37 & 30 \\
\hline H-02-03 & 17 & \\
\hline TOTAL & & \\
\hline 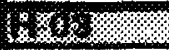 & r & \\
\hline H-03-01 & 5) & \\
\hline H03.02 & 25) & \\
\hline 11-03-03 & 6 & \\
\hline H-03-04 & 25 & \\
\hline H-03-05 & 7 & 13,6 \\
\hline 4-03-06 & 3 & \\
\hline TOTAL & & \\
\hline $18 \times 4 \%$ & & \\
\hline H-04-01 & 3 & \\
\hline $\mathrm{H}-04-02$ & 22 & \\
\hline $\mathrm{H}-0403$ & 9 & \\
\hline H-04-04 & 36 & \\
\hline H-04-05 & 6 & \\
\hline 1-04-06 & 22 & \\
\hline H-04-07 & 8 & \\
\hline H-04-08 & 201 & \\
\hline H-04-09 & 3 & \\
\hline H-04-10 & 26 & \\
\hline $\mathrm{H}-04-11$ & 19 & \\
\hline $14-04-12$ & 8 & \\
\hline H-04-13 & 16 & \\
\hline $\mathrm{H}-04-14$ & 13 & \\
\hline H-O\&-15 & 6 & 14,47 \\
\hline H-04-16 & 7 & \\
\hline TOTAL & & \\
\hline $1386 \%$ & sis & \\
\hline H-05-01 & 15 & \\
\hline H-05-02 & 33 & \\
\hline H-05-03 & 5 & \\
\hline H-05-04 & 9 & 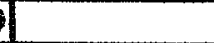 \\
\hline H-05-05 & 5! & 13,4 \\
\hline H-05-06 & 34 & \\
\hline TOTAL & & \\
\hline 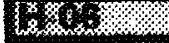 & & \\
\hline H-06-01 & 7 & \\
\hline H-06-02 & 30 & \\
\hline
\end{tabular}




\begin{tabular}{|c|c|c|}
\hline$F-05-05$ & 25 & \\
\hline$F-05-06$ & 41 & \\
\hline$F-05-07$ & 28 & \\
\hline$F-05-08$ & 10 & 22,56 \\
\hline$F-05-09$ & 14 & \\
\hline \multicolumn{3}{|l|}{ TOTAL } \\
\hline \multicolumn{3}{|l|}{ 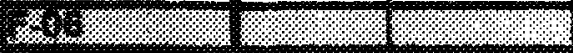 } \\
\hline $1-06-01$ & 4 & \\
\hline F-06-02 & 4 & \\
\hline$F-06-03$ & 27 & \\
\hline$F=06-04$ & 40 & \\
\hline F-06-05 & 36 & \\
\hline$F-06-06$ & 76 & \\
\hline F-06-07 & 80 & 38,14 \\
\hline$F-06-08$ & 59 & \\
\hline \multicolumn{3}{|l|}{ TOTAL } \\
\hline \multicolumn{3}{|l|}{ \% } \\
\hline$F-07-0 t$ & 1 & \\
\hline$F-07-02$ & 56 & \\
\hline$F=07-03$ & 10 & \\
\hline F-07-04 & 12 & \\
\hline$F-07-05$ & 24 & \\
\hline$F-07-06$ & 76 & \\
\hline$F-07-07$ & 40 & 31,29 \\
\hline$F-07-08$ & 38 & \\
\hline \multicolumn{3}{|l|}{ TOTAL } \\
\hline \multicolumn{3}{|l|}{ 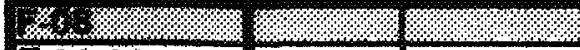 } \\
\hline$F-08-01$ & 21 & \\
\hline F-08-02 & 29 & \\
\hline$F-08-03$ & 10 & \\
\hline F-08-04 & 41 & \\
\hline$F-08-05$ & 2 & 20,6 \\
\hline F-08-06 & 65 & \\
\hline \multicolumn{3}{|l|}{ foral } \\
\hline \multicolumn{3}{|l|}{ 6y } \\
\hline$[F-09-01$ & 3 & \\
\hline F-09-02 & 30 & \\
\hline$F-09-03$ & 7 & \\
\hline$F=09-04$ & 46 & \\
\hline$F-09-05$ & 7 & 18,6 \\
\hline F-09-06 & 16 & \\
\hline \multicolumn{3}{|l|}{ IOTAL } \\
\hline \multicolumn{3}{|l|}{ \& } \\
\hline$F-10-01$ & 1 & \\
\hline$F-10-02$ & 12 & \\
\hline$F-10-03$ & 26 & \\
\hline$F-10-04$ & 18 & \\
\hline$F-10-05$ & 87 & 28,8 \\
\hline$F-10-06$ & 68 & \\
\hline TOTAL & & \\
\hline
\end{tabular}

\begin{tabular}{|c|c|c|}
\hline H-06-03 & 20 & \\
\hline H-06-04 & 15 & \\
\hline H-06-05 & 24 & \\
\hline H-06-0s & 14 & \\
\hline H-06-07 & 5 & 16,43 \\
\hline 1-06-08 & 8) & \\
\hline \multicolumn{3}{|l|}{ FOTAL } \\
\hline \multicolumn{3}{|l|}{ 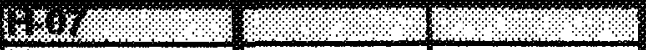 } \\
\hline H-07-01 & 1 & \\
\hline H-07-02 & 3 & \\
\hline H-07-03 & 21 & \\
\hline H-07-04 & 8 & \\
\hline $\mathrm{H}-07-05$ & 27 & \\
\hline $\mathrm{H}-07-06$ & 10 & \\
\hline H-07-07 & 6 & 10,86 \\
\hline H-07-08 & 28 & \\
\hline \multicolumn{3}{|l|}{ TOTAL } \\
\hline \multicolumn{3}{|l|}{ 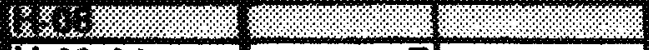 } \\
\hline $\mathrm{H}-08-01$ & 7 & \\
\hline $\mathrm{H}-08-02$ & 26 & \\
\hline H-08-03 & 19 & \\
\hline H-08-04 & 15 & \\
\hline H-08-05 & 16 & \\
\hline H-08-06 & 22 & \\
\hline H-08-07 & 34 & \\
\hline H-08-08 & 8 & \\
\hline $\mathrm{H}-08-09$ & 3 & \\
\hline H-08-10 & 21 & 17,1 \\
\hline $\mid+08-11$ & 12 & \\
\hline \multicolumn{3}{|l|}{ TOTAL } \\
\hline \multicolumn{3}{|l|}{ 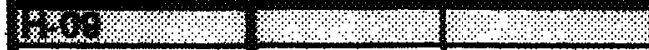 } \\
\hline H.09-01 & 12 & \\
\hline H-09-02 & 14 & \\
\hline H-09-03 & 5 & \\
\hline H-09-04 & 21 & 13 \\
\hline H-09-05 & 21 & \\
\hline \multicolumn{3}{|l|}{ TOTAL } \\
\hline \multicolumn{3}{|l|}{ (x) } \\
\hline H-10-01 & 4 & \\
\hline H-10-02 & 19 & \\
\hline $\mathrm{H}-10-03$ & 21 & \\
\hline $\mathrm{H}-10-04$ & 26 & \\
\hline H-10-05 & 21 & \\
\hline H-10-06 & 11 & 17 \\
\hline H-10-07 & 4 & \\
\hline TOTAL & & \\
\hline
\end{tabular}




\begin{tabular}{|c|c|c|c|c|c|}
\hline & \multicolumn{4}{|c|}{$\begin{array}{l}\text { Longueur des énoncés finals par rapport a la longueur } \\
\text { moyenne en syllabes des énoncés intra-tours }\end{array}$} & \\
\hline & enoncé final & énoncéss & & & \\
\hline & & intra-tours & & & intra-tours \\
\hline H-O & 4 & 37,333 & F-01 & 45 & 18,077 \\
\hline 14-02 & 17 & 30 & $F-02$ & 12 & 23,833 \\
\hline $4-03$ & 3 & 13,6 & $\mathrm{~F}-03$ & 54 & 24,667 \\
\hline $\mathrm{H}-04$ & 7 & 14,467 & $\overline{F-04}$ & 6 & 35,125 \\
\hline H-05 & 34 & 13,4 & $F-05$ & 14 & 22,556 \\
\hline H-06 & 8 & 16,429 & $F-06$ & 59 & 38,143 \\
\hline H.07 & 28 & 10,857 & F-07 & 38 & 31,286 \\
\hline $\mathrm{H}-08$ & 12 & 17,1 & $F-08$ & 65 & 20,6 \\
\hline $\mathrm{H}-0 \mathrm{O}$ & 21 & 13 & F-09 & 16 & 18,6 \\
\hline H-10 & 4 & 17 & $F-10$ & 68 & 28,8 \\
\hline Moyenne & 13,8 & 18,3186 & 3 Moyenne & 37,7 & 26,1687 \\
\hline
\end{tabular}




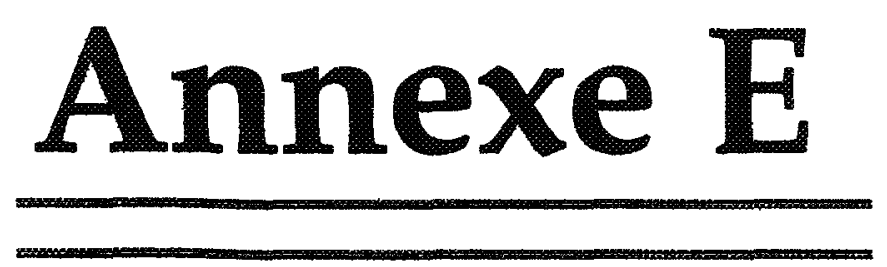

Tableau des indices de conclusion 


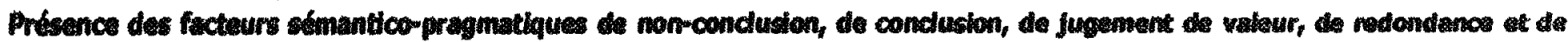

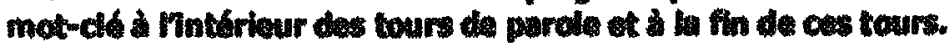

\begin{tabular}{|c|c|c|c|c|c|}
\hline Enonce du tour & Non-conclustion & Conclusion & Jugernent de valeu & Redandance & $\begin{array}{l}\text { Signaux explicites } \\
\text { inallo ou mot de }\end{array}$ \\
\hline $\mathrm{H}-01-02$ & 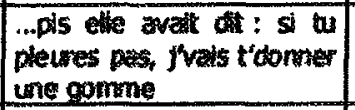 & & & & \\
\hline $\mathrm{H}-01 \mathrm{03}$ & & 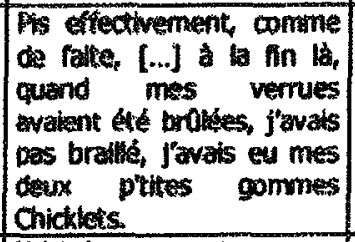 & & & \\
\hline $\mathrm{H}=01-04$ & & Trads ben content & & & \\
\hline$H-02-02 a$ & $\begin{array}{l}\text { quand } y \text { a des omautes } \\
\text { pls quy aretent des gens } \\
\text { la }\end{array}$ & & & & \\
\hline $\mathrm{H}-02-02 \mathrm{C}$ & & $\begin{array}{l}\text { If cistine, y sa font battre } \\
\text { waiment daplomb }\end{array}$ & & & \\
\hline $\mathrm{H}-02-03$ & & & & 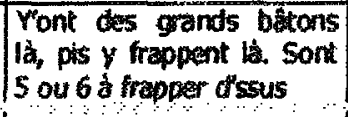 & \\
\hline $\mathrm{H}-03-02 \mathrm{a}$ & Sais-tu que c'matin la... & & & & \\
\hline $\mathrm{H}-03-02 \mathrm{~b}$ & $\begin{array}{l}\text { Jtals dans la douche pots } \\
\text { J'me suss of : }\end{array}$ & & & & \\
\hline $\mathrm{H}-03-02 \mathrm{C}$ & bon & & & & \\
\hline $\mathrm{H}-03-03$ & $\begin{array}{l}\text { Quest-ce qúa va } \\
\text { midemander? }\end{array}$ & & & & \\
\hline $\mathrm{H}-03.05$ & & JPavais préparé celle th & & & \\
\hline $4=03-06$ & & & C'est dommage. & & \\
\hline $\mathrm{H}-04-04$ & {$\left[\begin{array}{l}\text { Une balle qui messure à } \\
\text { peu prés } 3 \text { pouces de long } \\
\text { la }\end{array}\right.$} & & & & \\
\hline$H-04-06 a$ & Falt que on passe a coté & & & & \\
\hline
\end{tabular}




\begin{tabular}{|c|c|c|c|c|c|}
\hline Enoncel du lour & Nan-condusion & Conclusion & Iupenent de valeder & Redendance & 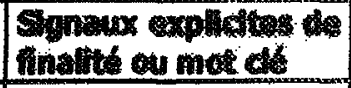 \\
\hline & dun chane & & & & \\
\hline $\mathrm{H}-04-06 \mathrm{~b}$ & Dans ce champ la & & & & \\
\hline H-04-07a-b & $\begin{array}{l}\text { Fat que varribte, pis y } \\
\text { minstate }\end{array}$ & & & & \\
\hline H-04-08 & Pis vawit fustermant... & & & & \\
\hline $\mathrm{H}-0409$ & & & & Y minstalle & \\
\hline H-04-10 & & 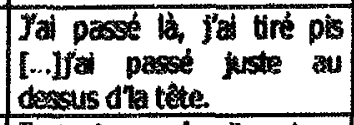 & & & \\
\hline$H=04-11$ & & 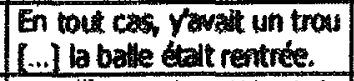 & & & \\
\hline$H-04-12$ & & $\begin{array}{l}\text { te silleux ext part tout de } \\
\text { sile. }\end{array}$ & & & \\
\hline $\mathrm{H}-04-13$ & & & 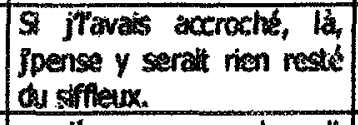 & & \\
\hline $\mathrm{H}-04-14$ & & & 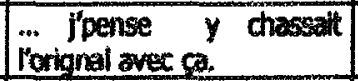 & $303 \ldots$ & \\
\hline H-04-15 & & & Euh. ftirais sun siffieux & & \\
\hline$H-04-16$ & & & & $\begin{array}{l}\text { Y srac pas resté grand } \\
\text { chose }\end{array}$ & \\
\hline$H-05-020$ & $\begin{array}{l}\text {.. y sprend de l'scorce de } \\
\text { boulezaul }\end{array}$ & & & & \\
\hline H.05.03 & & & Ca fall ccoentron. & & \\
\hline $\mathrm{H} 05.05$ & & & & Ga fat sper bon & \\
\hline H-05-06a & Pis... [allongement du pis] & & & & \\
\hline $\mathrm{H}-05-06 \mathrm{~b}$ & [...] le coned ba & & & & \\
\hline $\mathrm{H}-05-06 \mathrm{C}$ & & 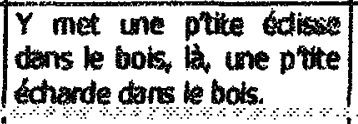 & & & \\
\hline$H-06-02 a$ & $\begin{array}{l}\text { Sats-u que } s a \text { fail } 5 \text { ans } \\
\text { an moins que Ime dis: }\end{array}$ & & & & \\
\hline$H .06 .04 a$ & As autivenentis & & & & \\
\hline
\end{tabular}




\begin{tabular}{|c|c|c|c|c|c|}
\hline Enoncé du tour & Non-condusion & Conctusion & Jugenent de valeu & Redondance & $\begin{array}{l}\text { Signaux oxpllotes de } \\
\text { fnstite cu mot dis }\end{array}$ \\
\hline H-06-06 & $\begin{array}{l}\text { L ct'ande fous pas } \\
\text { top une grosse session a } \\
\text { in la }\end{array}$ & & & & \\
\hline$H-06.07$ & & & Fiail que jonai profité. & & \\
\hline $\mathrm{H}-06-08$ & & & $\begin{array}{l}\text { Cest la premiere fois } \\
\text { ceppuis tongtemps. }\end{array}$ & & \\
\hline $\mathrm{H}-07-06$ & & & & $\begin{array}{l}\text { Tout le monde pensali } \\
\text { qu'les sucres étaient finis }\end{array}$ & \\
\hline $\mathrm{H}-07-07$ & & & & Tout le monda a détaillé. & \\
\hline$H-07-08 b$ & & $\begin{array}{l}\text { Mon oncle a attendu un } \\
\text { petu }[\text {...] y va avoir fatt } \\
\text { plusieurs gallons en fin de } \\
\text { semaine. }\end{array}$ & & & \\
\hline H-08-04 & Falt qu'on.. j’ai dit: & & & & \\
\hline $\mathrm{H}-08-05$ & $\begin{array}{l}\ldots \text { Iffaisais la non de lait } \\
\text { to... }\end{array}$ & & & & \\
\hline H-08-06 & $\begin{array}{l}\text {. déposer ma mère après } \\
\text { ca nü sour pis... }\end{array}$ & & & & \\
\hline $\mathrm{H}-08-07 a-b$ & & & & & 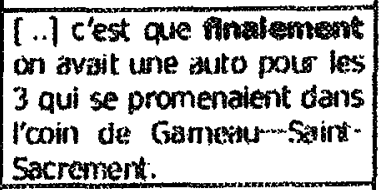 \\
\hline H-08-08 & & $\begin{array}{l}\text { Fis mon pere ivait laure } \\
\text { auto. }\end{array}$ & & & \\
\hline $\mathrm{H}-08-09$ & & latt que c'est ça. & & & \\
\hline $\mathrm{H}-08-11 \mathrm{~b}$ & & \begin{tabular}{|l|}
$\ldots$ j'aurtis eu du fun darns \\
leur cours.
\end{tabular} & & & \\
\hline $\mathrm{H}-09-04$ & & & & $\mid$\begin{tabular}{|l}
..tu peux pas vaiment te \\
planter, t'as difa prestion.
\end{tabular} & \\
\hline$H-10-03 a$ & {$\left[\begin{array}{l}\text {.. si tu fais oach quil est } \\
\text { pas correcte la }\end{array}\right.$} & & & & \\
\hline$H-10-04$ & $\begin{array}{l}\text { Par contre, tu sals que si } \\
\text { w fais goxh.. }\end{array}$ & & & & \\
\hline
\end{tabular}




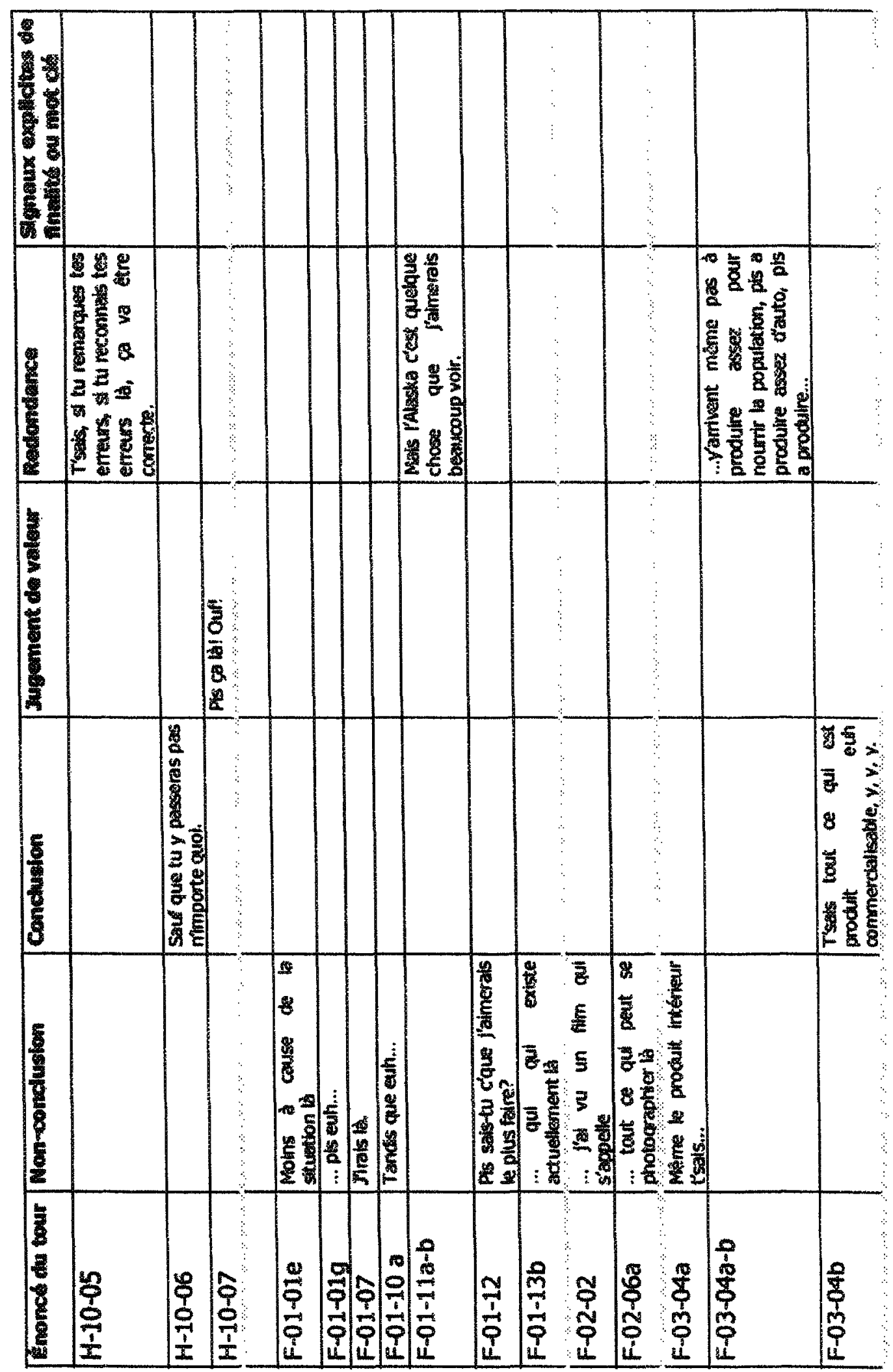




\begin{tabular}{|c|c|c|c|c|c|}
\hline Enonce du bour & Mon-conclusion & Conclusion & Jugement de veleur & Redondence & 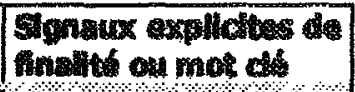 \\
\hline$F-04-01$ & {$\left[\begin{array}{l}\text { An! Cest une longue } \\
\text { Wistoire } \\
\text { Calédonte. }\end{array}\right.$} & & & & \\
\hline$F-04-04 a$ & 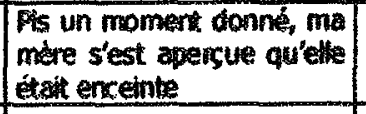 & & & & \\
\hline $\mathrm{F}-0404 \mathrm{~b}$ & & $\begin{array}{l}\text { Ca falt que v'ont décide de } \\
\text { pas y aller. }\end{array}$ & & & \\
\hline $\mathrm{F}=04-05 \mathrm{~b}$ & 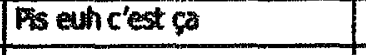 & & & & \\
\hline$F-04-08$ & & & & $\begin{array}{l}\text { Pis c'étalt vrainent } \\
\text { ayráable de Pentendre. }\end{array}$ & \\
\hline$F-04-09$ & & & & \begin{tabular}{|cc} 
retait pretre dons ce \\
temostl.
\end{tabular} & \\
\hline$F-05.02$ & & Ca talt cues.. & & & \\
\hline$F-05-03 a$ & E pis bon... & & & & \\
\hline$F-05-04$ & & & 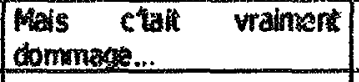 & & \\
\hline$F-05-07 c$ & & & & 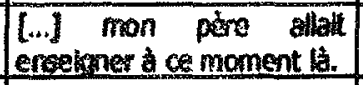 & \\
\hline$F-05-08$ & & & $\begin{array}{l}\text { Y'pense c'auralk gé blen } \\
\text { zasssi pour eun.. }\end{array}$ & & \\
\hline F-05-09 & & & & $\begin{array}{l}\text { Çauraik pautête the bien } \\
\text { pour mes parents.. }\end{array}$ & ...finatement \\
\hline$F-06-04 d$ & .... j'a pas dinconvenient is & & & & \\
\hline $\mathrm{F}-06-06 a$ & $\begin{array}{l}\text { Ce qui fall que des que le } \\
\text { vert se levalt }\end{array}$ & & & & \\
\hline$F-06-06 c$ & ..pistati & & & & \\
\hline$F-06-06 d$ & ... ten a partout ta & & & & \\
\hline$F \circ 06.06 e$ & & & 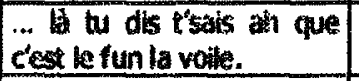 & & \\
\hline$F-06-07 a$ & 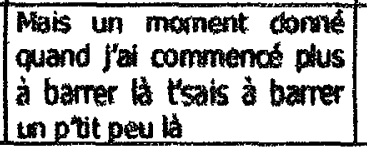 & & & & \\
\hline
\end{tabular}




\begin{tabular}{|c|c|c|c|c|c|}
\hline Enencé du tour & Non-condusion & Conclusion & Jugement de valeur & Redondance & $\begin{array}{l}\text { Signaux explicites do } \\
\text { inalite ou mot de }\end{array}$ \\
\hline$F=06-08 a$ & & & & & finalement.. \\
\hline$F-06-08 c$ & & & & & .finatement... \\
\hline$F-06-08 d-e-f$ & & & $\begin{array}{l}\text {... Pément intéressant de } \\
\text { la vale }\end{array}$ & & \\
\hline$F-07-02 b$ & $\begin{array}{l}\ldots \text { ave Lourence pis un } \\
\text { Brach allemand tí }\end{array}$ & & & & \\
\hline$F-07-06 a$ & $\begin{array}{l}\text { Puis auh..puis en floride } \\
\text { là, y laisaient presque } \\
\text { toupurs ces voyares la } \\
\end{array}$ & & & & \\
\hline$F-07-06 d$ & $\begin{array}{l}\ldots \text { 1a demiere fols qui en a } \\
\text { fatt un long la }\end{array}$ & & & & \\
\hline$F=07.07 a$ & Non & & & & \\
\hline$F-07-08$ & & & & & $\begin{array}{l}\text {..voyons pas les cớtes du } \\
\text { Maine mais euh... l'ettait } \\
\text { quil est juste en dessous } \\
\text { du Maine euh... corme ça } \\
\text { lá (quest. Indirecte) }\end{array}$ \\
\hline$F=08-05$ & & Fait que. & & & \\
\hline$\overline{F-08-06}$ & \begin{tabular}{|l|} 
Mais ga c'est des tas dos \\
royages de batezu là euh
\end{tabular} & & & & \\
\hline$F-09-04 a$ & ...j'vesex dire tu tu... & & & & \\
\hline$F-09-05$ & & & $\begin{array}{l}\text { Pis euh itrowe ca pesant } \\
\text { 'sais. }\end{array}$ & & \\
\hline$F-09-06 a-b$ & & & $\begin{array}{l}\text { Ypense que tout compte } \\
\text { fist on bien mieux ici } \\
\text { cu'aux Exats-Unis. }\end{array}$ & & \\
\hline$F-10-03 a$ & Cas fisit ase & & & & \\
\hline$F-10-03 b$ & ...j'a dit: & & & & \\
\hline $\mathrm{F}-10-04$ & Y'ont dat... & & & & \\
\hline$F-10-05$ & .pis là ... & & & & \\
\hline$F-10-06 a-b$ & Ga fait que là & & & & \\
\hline$F-10-06 h$ & & & 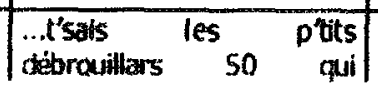 & & \\
\hline
\end{tabular}

